\title{
Differential $\{e\}$-structures for equivalences of 2-nondegenerate Levi rank 1 hypersurfaces $M^{5} \subset \mathbb{C}^{3}$
}

\author{
Wei GuO FOO AND JOËL MERKER*
}

\begin{abstract}
The class $\mathrm{IV}_{2}$ of 2-nondegenerate constant Levi rank 1 hypersurfaces $M^{5} \subset \mathbb{C}^{3}$ is governed by Pocchiola's two primary invariants $W_{0}$ and $J_{0}$. Their vanishing characterizes equivalence of such a hypersurface $M^{5}$ to the tube $M_{\mathrm{LC}}^{5}$ over the real light cone in $\mathbb{R}^{3}$. When either $W_{0} \not \equiv 0$ or $J_{0} \not \equiv 0$, by normalization of certain two group parameters c and e, an invariant coframe can be built on $M^{5}$, showing that the dimension of the CR automorphism group drops from 10 to 5 .

This paper constructs an explicit $\{e\}$-structure in case $W_{0}$ and $J_{0}$ do not necessarily vanish. Furthermore, Pocchiola's calculations hidden on a computer now appear in details, especially the determination of a secondary invariant $R$, expressed in terms of the first jet of $W_{0}$. All other secondary invariants of the $\{e\}$-structure are also expressed explicitly in terms of $W_{0}$ and $J_{0}$.
\end{abstract}

Keywords: Levi degenerate CR manifolds, 2-nondegeneracy, G-structures, Cartan method of equivalence, Cartan Lemma, Pocchiola invariants.

2020 Mathematics Subject Classification: 58A15, 53A55, 53B25, 53C10, 32V25, 32V40.

\section{INTRODUCTION}

We study the equivalence problem under biholomorphisms of real hypersurfaces $M^{5} \subset$ $\mathbb{C}^{3}$ - hence of CR dimension 2 - whose Levi form is degenerate of constant rank 1 , and whose Freeman form is nowhere zero, or equivalently, which are 2-nondegenerate. There are previous approaches to this problem, and we refer our readers to the the articles of MedoriSpiro $[12,13]$, in which a Cartan connection was adressed.

In a recently published article [18], the authors exhibited two important primary invariants, $W_{0}$ and $J_{0}$, whose existence was not previously discovered prior to Pocchiola's prepublication [25], and which, in depth, required the help of a computer algebra system. These invariants have useful applications, such as in Isaev's study [9] of tube hypersurfaces in $\mathbb{C}^{3}$ that are 2-nondegenerate and uniformly Levi degenerate of rank 1.

Our first objective here is to reconstruct $W_{0}$ and $J_{0}$, by presenting fully detailed computations, only by hand, without the help of any computer. In contrast to [25, 18], the present text has the ambition of exhibiting all calculations, without requiring any extra work from the readers: 'no pen needed, no computer needed'. Within the Cartan theory, this sounds quite like a challenge opposite to a certain tradition of hiding a lot of computations. But we believe that fully detailed articles can be read, checked and studied more rapidly.

As a second objective, we construct an explicit $\{e\}$-structure which characterizes equivalences under biholomorphisms of these types of hypersurfaces $M^{5} \subset \mathbb{C}^{3}$. This way, we give a

Received: 26.05.2021; Accepted: 25.08.2021; Published Online: 15.09.2021

*Corresponding author: Joël Merker; joel.merker@universite-paris-saclay.fr

DOI: $10.33205 / \mathrm{cma} .943426$ 
theoretical proof which will provide a definitive confirmation of the existence of exactly 2 primary invariants, $W_{0}$ and $J_{0}$. Unlike the approach of $[25,18]$ which proceeded at each step with systematic and explicit calculations of all torsion coefficients, we will bypass some of these steps, thereby economizing some computations. On the way, we will closely observe the evolution of the modified Maurer-Cartan 1-forms during the Cartan process.

The basic principle of Cartan's approach is to create a collection of 1-forms (a coframe), by absorbing as many as possible torsion terms, in order that the structure of this coframe be as close as possible to the structure of the Maurer-Cartan coframe on the (prolongation of the) model $M_{\mathrm{LC}}^{5} \subset \mathbb{C}^{3}$, the tube over the real light cone $\left\{x_{1}^{2}+x_{2}^{2}=x_{3}^{2}\right\}$ in $\mathbb{R}^{3}$ :

$$
M_{\mathrm{LC}}^{5}:=\left\{\left(z_{1}, z_{2}, z_{3}\right) \in \mathbb{C}^{3}:\left(\operatorname{Re} z_{1}\right)^{2}+\left(\operatorname{Re} z_{2}\right)^{2}=\left(\operatorname{Re} z_{3}\right)^{2}\right\}
$$

whose local CR automorphism group is known to be isomorphic to $\mathrm{SO}_{3,2}(\mathbb{R})$.

Recall that a Maurer-Cartan form $\omega$ valued in some Lie algebra $\mathfrak{g}$ satisfies the structure equation with no curvature:

$$
d \omega+\frac{1}{2}[\omega \wedge \omega]=0 .
$$

In practice, as in our current case, the right-hand side of the equation is not always zero, and this constitutes the default of $\omega$ being a Maurer-Cartan form. This happens when an invariant is written as a linear combination of torsion terms, and such a linear combination fails to follow the structure equations, thus obstructing the absorption process.

We now give a summary of our results. Recall that if $J$ denotes the complex structure of $T \mathbb{C}^{3}$, then the tangent bundle $T M^{5}$ has a distribution $T^{c} M^{5}:=T M^{5} \cap J T M^{5} \subseteq T M^{5}$ of codimension 1 which is invariant under $J$ at each point of $M^{5}$. Let $\rho$ be a real 1-form with Ker $\rho=T^{c} M^{5}$. The Levi form is a bilinear map on $T^{c} M^{5}$ defined as $(X, Y) \mapsto d \rho(X, J Y)$ for any two sections $X, Y$ of $T^{c} M^{5}$.

Letting $\mathbb{C} T M^{5}:=\mathbb{C} \otimes_{\mathbb{R}} T M^{5}$ be the complexification of the tangent bundle of $M^{5}$, by defining $T^{1,0} M^{5}:=\mathbb{C} T M^{5} \cap T^{1,0} \mathbb{C}^{3}$ together with its complex conjugate $T^{0,1} M^{5}:=\overline{T^{1,0} M^{5}}$, we have the (classical) direct sum decomposition $\mathbb{C} T^{c} M^{5}=T^{1,0} M^{5} \oplus T^{0,1} M^{5}$. Let $\left\{\mathcal{L}_{1}, \mathcal{L}_{2}\right\}$ be two local generators of $T^{1,0} M^{5}$, i.e. a frame for $T^{1,0} M^{5}$.

Section 2 provides more information, while complete background may be found in [19].

By the assumption that the Levi form is uniformly of rank 1 at each point of $M$, there exists by [19] a uniquely determined slant function $k: M \longrightarrow \mathbb{C}$ such that the vector field:

$$
\mathcal{K}:=k \mathcal{L}_{1}+\mathcal{L}_{2}
$$

generates the kernel of the Levi form, of constant rank $2-1=1$. If we let $\mathcal{T}$ denote a vector field with $\rho(\mathcal{T}) \equiv 1$, we may consider the coframe $\left\{\rho, \kappa_{0}, \zeta_{0}\right\}$ dual to $\left\{\mathcal{T}, \mathcal{L}_{1}, \mathcal{K}\right\}$. In fact, the conjugates $\bar{\kappa}_{0}, \bar{\zeta}_{0}$ and $\overline{\mathcal{L}}_{1}, \overline{\mathcal{K}}$ also come into play in order to really make up a (co)frame on $\mathbb{C} T M^{5}$, while $\bar{\rho}=\rho$ and $\overline{\mathcal{T}}=\mathcal{T}$ are real. A certain appropriate real 1-form $\rho$ will be chosen, and denoted $\rho_{0}$.

Performing the Cartan process, we will make a series of changes to these 1-forms:

$$
\left(\rho_{0}, \kappa_{0}, \zeta_{0}\right) \rightsquigarrow\left(\rho_{0}, \kappa_{0}^{\prime}, \zeta_{0}^{\prime \prime}\right)
$$

and after (really a lot of) computations, we will obtain a 4-dimensional G-structure whose lifted 1-forms write up as:

$$
\left(\begin{array}{l}
\rho \\
\kappa \\
\zeta
\end{array}\right):=\left(\begin{array}{ccc}
c \bar{c} & 0 & 0 \\
-i \overline{\mathrm{c} e} & \mathrm{c} & 0 \\
-\frac{i}{2} \frac{\mathrm{ce} e}{\mathrm{c}} & \mathrm{e} & \frac{\mathrm{c}}{\bar{c}}
\end{array}\right)\left(\begin{array}{c}
\rho_{0} \\
\kappa_{0}^{\prime} \\
\zeta_{0}^{\prime \prime}
\end{array}\right)
$$


Also, after a long process, we will construct modified Maurer-Cartan forms:

$$
\begin{aligned}
& \pi^{1}:=\alpha-\left(\mathrm{t}-\frac{i}{2} \operatorname{Im} Z^{2}\right) \rho-\left(R^{1}-\bar{K}^{6}\right) \kappa-R^{2} \zeta-K^{6} \bar{\kappa}-0 \\
& \pi^{2}:=\beta-i Z^{1} \rho-\left(\mathrm{t}-\frac{i}{2} \operatorname{lm} Z^{2}+K^{1}\right) \kappa-K^{2} \zeta-K^{3} \bar{\kappa}-K^{4} \bar{\zeta}
\end{aligned}
$$

with $R^{i}, K^{i}, Z^{i}$ being some explicit functions on $M^{5} \times G^{4}$, where $\mathrm{t}$ is a new real variable, and then, after meticulous absorption work, we will obtain as is stated below in Theorem 13.1 on p. 366, three finalized structure equations of the neat shape:

$$
\begin{aligned}
d \rho= & \left(\pi^{1}+\bar{\pi}^{1}\right) \wedge \rho+i \kappa \wedge \bar{\kappa}, \\
d \kappa= & \pi^{2} \wedge \rho+\pi^{1} \wedge \kappa+\zeta \wedge \bar{\kappa} \\
d \zeta= & \left(\pi^{1}-\bar{\pi}^{1}\right) \wedge \zeta+i \pi^{2} \wedge \kappa \\
& +R \rho \wedge \zeta+J \rho \wedge \bar{\kappa}+W \kappa \wedge \zeta,
\end{aligned}
$$

in which are present Pocchiola's two primary invariants:

$$
W=\frac{1}{\mathrm{c}} W_{0} \quad \text { and } \quad J=\frac{i}{\overline{\mathrm{c}}^{3}} \bar{J}_{0},
$$

together with a single secondary (derived) invariant:

$$
R=\operatorname{Re}\left[i \frac{\mathrm{e}}{\mathrm{cc}} W_{0}+\frac{1}{\mathrm{c} \overline{\mathrm{C}}}\left(-\frac{i}{2} \overline{\mathcal{L}}_{1}\left(W_{0}\right)+\frac{i}{2}\left(-\frac{1}{3} \frac{\overline{\mathcal{L}}_{1}\left(\overline{\mathcal{L}}_{1}(k)\right)}{\overline{\mathcal{L}}_{1}(k)}+\frac{1}{3} \bar{P}\right) W_{0}\right)\right]
$$

We would like to mention that the two invariants that Pocchiola denoted $W$ and $J$ are now denoted in our paper $W_{0}$ and $J_{0}$, with the subscript $(\bullet)_{0}$ designating functions defined on $M^{5}$ alone, independently of any extra group variable.

The expression of $R$ was discovered by Pocchiola in $[25,18]$ thanks to intensive computer explorations, but no details of proof appeared in print at all. In Section 12 of this paper, a complete, detailed, hand-done proof, will be provided, thus verifying that $R$ is indeed a function of the first jet of $W_{0}$, hence a secondary invariant.

We will also construct a certain real 1-form $\Lambda=d \mathrm{t}+\cdots$, and in Section 14, the final $\{e\}$ structure that we obtain will take the following form (conjugate equations are unwritten):

$$
\begin{aligned}
d \rho & =\pi^{1} \wedge \rho+\bar{\pi}^{1} \wedge \rho+i \kappa \wedge \bar{\kappa} \\
d \kappa & =\pi^{1} \wedge \kappa+\pi^{2} \wedge \rho+\zeta \wedge \bar{\kappa} \\
d \zeta & =i \pi^{2} \wedge \kappa+\pi^{1} \wedge \zeta-\bar{\pi}^{1} \wedge \zeta+W \kappa \wedge \zeta+R \rho \wedge \zeta+J \rho \wedge \bar{\kappa} \\
d \pi^{1} & =\Lambda \wedge \rho-i \bar{\pi}^{2} \wedge \kappa+\zeta \wedge \bar{\zeta}+\widehat{\Omega}_{1} \\
d \pi^{2} & =\Lambda \wedge \kappa+\pi^{2} \wedge \bar{\pi}^{1}-\bar{\pi}^{2} \wedge \zeta+\widehat{\Omega}_{2}+h \rho \wedge \kappa \\
d \Lambda & =\Lambda \wedge \pi^{1}+\Lambda \wedge \bar{\pi}^{1}+i \pi^{2} \wedge \bar{\pi}^{2}+\Phi
\end{aligned}
$$


with

$$
\begin{aligned}
\widehat{\Omega}_{1}= & -\frac{1}{4} W \pi^{2} \wedge \rho+\frac{1}{4} \bar{W} \bar{\pi}^{2} \wedge \rho-\frac{1}{2}\left(R_{\kappa}-\overline{J_{\zeta}}\right) \rho \wedge \kappa-\frac{1}{2} R_{\zeta} \rho \wedge \zeta \\
& +\frac{1}{2}\left(R_{\bar{\kappa}}-J_{\zeta}\right) \rho \wedge \bar{\kappa}+\frac{1}{2} R_{\bar{\zeta}} \rho \wedge \bar{\zeta}+\left(\frac{1}{2} W_{\bar{\kappa}}-i R\right) \kappa \wedge \bar{\kappa}-\bar{W} \kappa \wedge \bar{\zeta}-W \zeta \wedge \bar{\kappa}, \\
\widehat{\Omega}_{2}= & -R \pi^{2} \wedge \rho-\frac{1}{4} W \pi^{2} \wedge \kappa+\frac{1}{4} \bar{W} \bar{\pi}^{2} \wedge \kappa-i\left(W_{\rho}-2 R_{\kappa}+\overline{J_{\zeta}}\right) \rho \wedge \zeta \\
& -i\left(W J-J_{\kappa}\right) \rho \wedge \bar{\kappa}-i J \rho \wedge \bar{\zeta}-\frac{1}{2} R_{\zeta} \kappa \wedge \zeta+\frac{1}{2}\left(R_{\bar{\kappa}}-J_{\zeta}\right) \kappa \wedge \bar{\kappa}+\frac{1}{2} R_{\bar{\zeta}} \kappa \wedge \bar{\zeta} \\
& -R \zeta \wedge \bar{\kappa} .
\end{aligned}
$$

Furthermore, we will show that $h$ and $\Phi$ can be expressed in terms of $\widehat{\Omega}_{1}$, of $\widehat{\Omega}_{2}$ and of their first-order derivatives. Thus, this demonstrates that there are exactly 2 primary invariants.

Clearly, when $W \equiv J \equiv 0$, the $\{e\}$-structure collapses to:

$$
\begin{aligned}
d \rho & =\pi^{1} \wedge \rho+\bar{\pi}^{1} \wedge \rho+i \kappa \wedge \bar{\kappa}, \\
d \kappa & =\pi^{1} \wedge \kappa+\pi^{2} \wedge \rho+\zeta \wedge \bar{\kappa}, \\
d \zeta & =i \pi^{2} \wedge \kappa+\pi^{1} \wedge \zeta-\bar{\pi}^{1} \wedge \zeta, \\
d \pi^{1} & =\Lambda \wedge \rho-i \bar{\pi}^{2} \wedge \kappa+\zeta \wedge \bar{\zeta}, \\
d \pi^{2} & =\Lambda \wedge \kappa+\pi^{2} \wedge \bar{\pi}^{1}-\bar{\pi}^{2} \wedge \zeta, \\
d \Lambda & =\Lambda \wedge \pi^{1}+\Lambda \wedge \bar{\pi}^{1}+i \pi^{2} \wedge \bar{\pi}^{2}
\end{aligned}
$$

and these constant coefficients equations correspond to the structure equations of the tube $M_{\mathrm{LC}}^{5}$ over the light cone, which is the reference model for this equivalence problem.

We would like to mention that, strictly speaking, Cartan's equivalence method of producing homogeneous models requires to normalize any group variable which occurs in some essential torsion term, and this is what Pocchiola did in Section 7 of [25] for $c:=\left(\boldsymbol{J}_{0}\right)^{1 / 3}$ and in Section 8 for $c:=W_{0}$, showing afterwards that e can also be normalized in both cases.

For this deep reason, Pocchiola then disregarded the - essentially useless - task of constructing a general $\{e\}$-structure, since, when $J_{0} \equiv W_{0} \equiv 0$, the final Section 9 of [25] shows that one comes uniquely to the structure equations of the model $M_{\mathrm{LC}}^{5}$, without any further nonzero essential torsion appearing. And this was really a discovery, because most of the times in CR geometry, primary invariants appear after a first prolongation.

However, because there is a tradition of setting up $\{e\}$-structures, even in absence of explicit computations, even without discovering invariants at all, and because the needs for verifiable computations has been expressed by some experts, we decided to set up the present article. While re-building this chapter [25] of Pocchiola's Ph.D. (Orsay University, September 2014), we found a few copying mistakes in some intermediate formulas of $[25,18]$, but no error in either statements or final formulas, e.g. $W_{0}$ and $J_{0}$ are correct.

For a more informative exposition of introductory aspects, the reader should read now the brief and complementary Introduction to the Addendum to [18].

This paper is organized as follows. In Section 2, we recall the local geometry of 2-nondegenerate Levi rank 1 real hypersurfaces $M^{5}$ in $\mathbb{C}^{3}$. In Section 3, we give a description of the $G_{1}$-structure of the biholomorphic equivalences of such real hypersurfaces. Section 4 gives a quick glimpse of a series of normalizations of parameters, which will be detailed in Sections 5 to 10, with the first appearance of $W_{0}$ in Section 8. The explicit expression of the invariant $J_{0}$ is given in Section 11, and a complete proof of the above formula for $R$ is detailed in Section 12. Section 13 gives a short summary of the things that have been done in the previous sections, and finally Section 14 gives a proposed $\{e\}$-structure for the equivalence problem. 
2. LOCAL GEOMETRY OF 2-NONDEGENERATE LEVI RANK 1 HYPERSURFACES $M^{5} \subset \mathbb{C}^{3}$

This section only summarizes what has been presented and detailed in $[19,17,18]$. Let $M^{5} \subset \mathbb{C}^{3}$ be a $\mathcal{C}^{\omega}$ (real-analytic) smooth, local or global, real hypersurface and let $p_{0} \in M$. In any affine holomorphic coordinate system:

$$
\left(z_{1}, z_{2}, w\right) \in \mathbb{C}^{3} \quad \text { with } \quad w=u+i v,
$$

centered at $p_{0}=(0,0,0)=0$ in which $\left.\frac{\partial}{\partial u}\right|_{0} \notin T_{0} M$, there is a local $\mathcal{C}^{\omega}$ graphing function $F=F\left(z_{1}, z_{2}, \bar{z}_{1}, \bar{z}_{2}, v\right)$ with $F(0)=0$ such that $M$ is represented, in some (possibly small) open neighborhood of the origin 0 by

$$
u=F\left(z_{1}, z_{2}, \bar{z}_{1}, \bar{z}_{2}, v\right) .
$$

Convention 2.1. From now on, the hypersurface will be identified with its localization in some small open neighborhood of the origin, and it will always be denoted by $M$.

As is known (see [19] for detailed background), the complexified tangent bundle $\mathbb{C} T M:=$ $\mathbb{C} \otimes_{\mathbb{R}} T M$ inherits from $\mathbb{C} T \mathbb{C}:=\mathbb{C} \otimes_{\mathbb{R}} T \mathbb{C}^{3}$ two biholomorphically invariant complex rank 2 vector subbundles

$$
T^{1,0} M:=T^{1,0} \mathbb{C}^{3} \cap \mathbb{C} T M \quad \text { and } \quad T^{0,1} M:=T^{0,1} \mathbb{C}^{3} \cap \mathbb{C} T M=\overline{T^{1,0} M}
$$

which are conjugate one to another. Then a check shows that the two vector fields written in the intrinsic coordinates $\left(z_{1}, z_{2}, \bar{z}_{1}, \bar{z}_{2}, v\right)$ on $M$ :

$$
\mathcal{L}_{1}:=\frac{\partial}{\partial z_{1}}+A^{1} \frac{\partial}{\partial v} \quad \text { and } \quad \mathcal{L}_{2}:=\frac{\partial}{\partial z_{2}}+A^{2} \frac{\partial}{\partial v},
$$

whose coefficients are defined by:

$$
A^{i}:=-i \frac{F_{z_{i}}}{1+i F_{v}} \quad(i=1,2),
$$

generate $T^{1,0} M$, locally. Hence their two conjugates $\overline{\mathcal{L}}_{1}, \overline{\mathcal{L}}_{2}$ generate the bundle $T^{0,1} M$, also of complex rank 2.

Then visibly the differential 1-form

$$
\varrho_{0}:=d v-A^{1} d z_{1}-A^{2} d z_{2}-\bar{A}^{1} d \bar{z}_{1}-\bar{A}^{2} d \bar{z}_{2}
$$

has kernel

$$
\left\{\varrho_{0}=0\right\}=T^{1,0} M \oplus T^{0,1} M .
$$

There are various (equivalent) aspects of the concept of Levi form of $M$, but they will not be recalled here, since several sources treat that. Here, the Levi form of $M$ can be represented as a function of the points

$$
p=\left(z_{1}, z_{2}, \bar{z}_{1}, \bar{z}_{2}, v\right) \in M,
$$

valued in the space of Hermitian $2 \times 2$ matrices, and in terms of $\varrho_{0}$ and of the Lie brackets of the above vector fields, it writes as

$$
\operatorname{LF}_{M}(p):=\left(\begin{array}{ll}
\varrho_{0}\left(i\left[\mathcal{L}_{1}, \overline{\mathcal{L}}_{1}\right]\right) & \varrho_{0}\left(i\left[\mathcal{L}_{2}, \overline{\mathcal{L}}_{1}\right]\right) \\
\varrho_{0}\left(i\left[\mathcal{L}_{1}, \overline{\mathcal{L}}_{2}\right]\right) & \varrho_{0}\left(i\left[\mathcal{L}_{2}, \overline{\mathcal{L}}_{2}\right]\right)
\end{array}\right)(p) .
$$

As is known, the biholomorphic invariance of the Levi form legitimates our current

Hypothesis 2.2. [Uniform Levi rank 1] At all points $p \in M$, the Levi matrix (form) $\operatorname{LF}_{M}(p$ ) has constant rank 1. 
After a linear change of coordinates in the $\left(z_{1}, z_{2}\right)$ space, we may assume that its $(1,1)$-entry vanishes nowhere on $M$ :

$$
\varrho_{0}\left(i\left[\mathcal{L}_{1}, \overline{\mathcal{L}}_{1}\right]\right)(p) \neq 0 \quad(\forall p \in M) .
$$

This means that the real vector field

$$
\mathcal{T}:=i\left[\mathcal{L}_{1}, \overline{\mathcal{L}}_{1}\right]=i\left(\mathcal{L}_{1}\left(\bar{A}^{1}\right)-\overline{\mathcal{L}}_{1}\left(A^{1}\right)\right) \frac{\partial}{\partial v}=: \ell \frac{\partial}{\partial v}
$$

has nowhere vanishing real coefficient that will be abbreviated as

$$
\ell:=i\left(\bar{A}_{z_{1}}^{1}+A^{1} \bar{A}_{v}^{1}-A_{\bar{z}_{1}}^{1}-\bar{A}^{1} A_{v}^{1}\right) \neq 0 .
$$

Furthermore, since the $2 \times 2$ Levi matrix has constant rank 1 , the collection of its 1-dimensional kernels at all points $p \in M$ spans a $\mathcal{C}^{\omega}$ smooth subdistribution $K^{1,0} M \subset T^{1,0} M$ which satisfies ([19], pp. 72-73):

$$
\begin{aligned}
& {\left[K^{1,0} M, K^{1,0} M\right] \subset K^{1,0} M,} \\
& {\left[K^{0,1} M, K^{0,1} M\right] \subset K^{0,1} M,} \\
& {\left[K^{1,0} M, K^{0,1} M\right] \subset K^{1,0} M \oplus K^{0,1} M \quad\left(K^{0,1} M:=\overline{K^{1,0} M}\right) .}
\end{aligned}
$$

With this, a vector field generator $\mathcal{K}$ of $K^{1,0} M$ writes uniquely as

$$
\mathcal{K}:=k \mathcal{L}_{1}+\mathcal{L}_{2},
$$

where the function $k$ - very important in the theory - is the negative of the quotient of two entries of the Levi matrix

$$
k:=-\frac{\mathcal{L}_{2}\left(\bar{A}^{1}\right)-\overline{\mathcal{L}}_{1}\left(A^{2}\right)}{\mathcal{L}_{1}\left(\bar{A}^{1}\right)-\overline{\mathcal{L}}_{1}\left(A^{1}\right)} .
$$

Hypothesis 2.3. [2-nondegeneracy] At all points $p \in M$, the Freeman form has constant (maximal possible) rank 1.

For a detailed presentation of this second concept of form, also biholomorphically invariant, see [19].

Proposition 2.4. ([19]) In this formalism, $M$ is 2-nondegenerate if and only if:

$$
\overline{\mathcal{L}}_{1}(k) \neq 0 \quad(\text { everywhere on } M) .
$$

In summary, two functions will be assumed to be nowhere vanishing on $M$, corresponding to the two Hypotheses 2.2 and 2.3:

$$
\ell(p) \neq 0 \quad \text { and } \quad \overline{\mathcal{L}}_{1}(k)(p) \neq 0 \quad(\forall p \in M) .
$$

Next, along with $k$, introduce a second and last fundamental function

$$
P:=\frac{\ell_{z_{1}}+A^{1} \ell_{v}-\ell A_{v}^{1}}{\ell}
$$

All invariants and semi-invariants in this paper will express in terms of $k$ and $P$. 
Next, according to $[17,25,18]$, there are 10 Lie bracket identities

$$
\begin{aligned}
{\left[\mathcal{T}, \mathcal{L}_{1}\right] } & =-P \cdot \mathcal{T} \\
{[\mathcal{T}, \mathcal{K}] } & =\mathcal{L}_{1}(k) \cdot \mathcal{T}+\mathcal{T}(k) \cdot \mathcal{L}_{1}, \\
{\left[\mathcal{T}, \overline{\mathcal{L}}_{1}\right] } & =-\bar{P} \cdot \mathcal{T} \\
{[\mathcal{T}, \overline{\mathcal{K}}] } & =\overline{\mathcal{L}}_{1}(\bar{k}) \cdot \mathcal{T}+\mathcal{T}(\bar{k}) \cdot \overline{\mathcal{L}}_{1}, \\
{\left[\mathcal{L}_{1}, \mathcal{K}\right] } & =\mathcal{L}_{1}(k) \cdot \mathcal{L}_{1} \\
{\left[\mathcal{L}_{1}, \overline{\mathcal{L}}_{1}\right] } & =-i \mathcal{T}, \\
{\left[\mathcal{L}_{1}, \overline{\mathcal{K}}\right] } & =\mathcal{L}_{1}(\bar{k}) \cdot \overline{\mathcal{L}}_{1} \\
{\left[\mathcal{K}, \overline{\mathcal{L}}_{1}\right] } & =-\overline{\mathcal{L}}_{1}(k) \cdot \mathcal{L}_{1}, \\
{[\mathcal{K}, \overline{\mathcal{K}}] } & =0 \\
{\left[\overline{\mathcal{L}}_{1}, \overline{\mathcal{K}}\right] } & =\overline{\mathcal{L}}_{1}(\bar{k}) \cdot \overline{\mathcal{L}}_{1} .
\end{aligned}
$$

Lemma 2.5. ([19, 17]) The following 3 functional identities hold identically on $M$.

$$
\begin{aligned}
\mathcal{K}(\bar{k}) & \equiv 0, \\
\mathcal{K}(P) & \equiv-P \mathcal{L}_{1}(k)-\mathcal{L}_{1}\left(\mathcal{L}_{1}(k)\right), \\
\mathcal{K}(\bar{P}) & \equiv-P \overline{\mathcal{L}}_{1}(k)-\overline{\mathcal{L}}_{1}\left(\mathcal{L}_{1}(k)\right)-i \mathcal{T}(k) .
\end{aligned}
$$

Then the coframe

$$
\left\{\rho_{0}, \kappa_{0}, \zeta_{0}, \bar{\kappa}_{0}, \bar{\zeta}_{0}\right\}
$$

dual to the frame

$$
\left\{\mathcal{T}, \mathcal{L}_{1}, \mathcal{K}, \overline{\mathcal{L}}_{1}, \overline{\mathcal{K}}\right\}
$$

i.e. which satisfies by definition

$$
\begin{aligned}
& \rho_{0}(\mathcal{T})=1, \quad \rho_{0}\left(\mathcal{L}_{1}\right)=0, \quad \rho_{0}(\mathcal{K})=0, \quad \rho_{0}\left(\overline{\mathcal{L}}_{1}\right)=0, \quad \rho_{0}(\overline{\mathcal{K}})=0, \\
& \kappa_{0}(\mathcal{T})=0, \quad \kappa_{0}\left(\mathcal{L}_{1}\right)=1, \quad \kappa_{0}(\mathcal{K})=0, \quad \kappa_{0}\left(\overline{\mathcal{L}}_{1}\right)=0, \quad \kappa_{0}(\overline{\mathcal{K}})=0, \\
& \zeta_{0}(\mathcal{T})=0, \quad \zeta_{0}\left(\mathcal{L}_{1}\right)=0, \quad \zeta_{0}(\mathcal{K})=1, \quad \zeta_{0}\left(\overline{\mathcal{L}}_{1}\right)=0, \quad \zeta_{0}(\overline{\mathcal{K}})=0, \\
& \bar{\kappa}_{0}(\mathcal{T})=0, \quad \bar{\kappa}_{0}\left(\mathcal{L}_{1}\right)=0, \quad \bar{\kappa}_{0}(\mathcal{K})=0, \quad \bar{\kappa}_{0}\left(\overline{\mathcal{L}}_{1}\right)=1, \quad \bar{\kappa}_{0}(\overline{\mathcal{K}})=0, \\
& \bar{\zeta}_{0}(\mathcal{T})=0, \quad \bar{\zeta}_{0}\left(\mathcal{L}_{1}\right)=0, \quad \bar{\zeta}_{0}(\mathcal{K})=0, \quad \bar{\zeta}_{0}\left(\overline{\mathcal{L}}_{1}\right)=0, \quad \bar{\zeta}_{0}(\overline{\mathcal{K}})=1,
\end{aligned}
$$

has its 5 component 1 -forms given explicitly by

$$
\begin{aligned}
\rho_{0} & =\frac{d v-A^{1} d z_{1}-A^{2} d z_{2}-\bar{A}^{1} d \bar{z}_{1}-\bar{A}^{2} d \bar{z}_{2}}{\ell}, \\
\kappa_{0} & =d z_{1}-k d z_{2}, \\
\zeta_{0} & =d z_{2}, \\
\bar{\kappa}_{0} & =d \bar{z}_{1}-\bar{k} d \bar{z}_{2}, \\
\bar{\zeta}_{0} & =d \bar{z}_{2} .
\end{aligned}
$$

Notice that a different notation $\rho_{0} \neq \varrho_{0}$ has been employed just now. Hence using a classical formula which goes back at least to Lie ([11, Chap. 5]) which holds for two arbitrary vector fields $X$ and $Y$ and for any differential 1-form $\omega$

$$
d \omega(X, Y)=X(\omega(Y))-Y(\omega(X))-\omega([X, Y]),
$$


by representing the 10 Lie brackets in some appropriate array

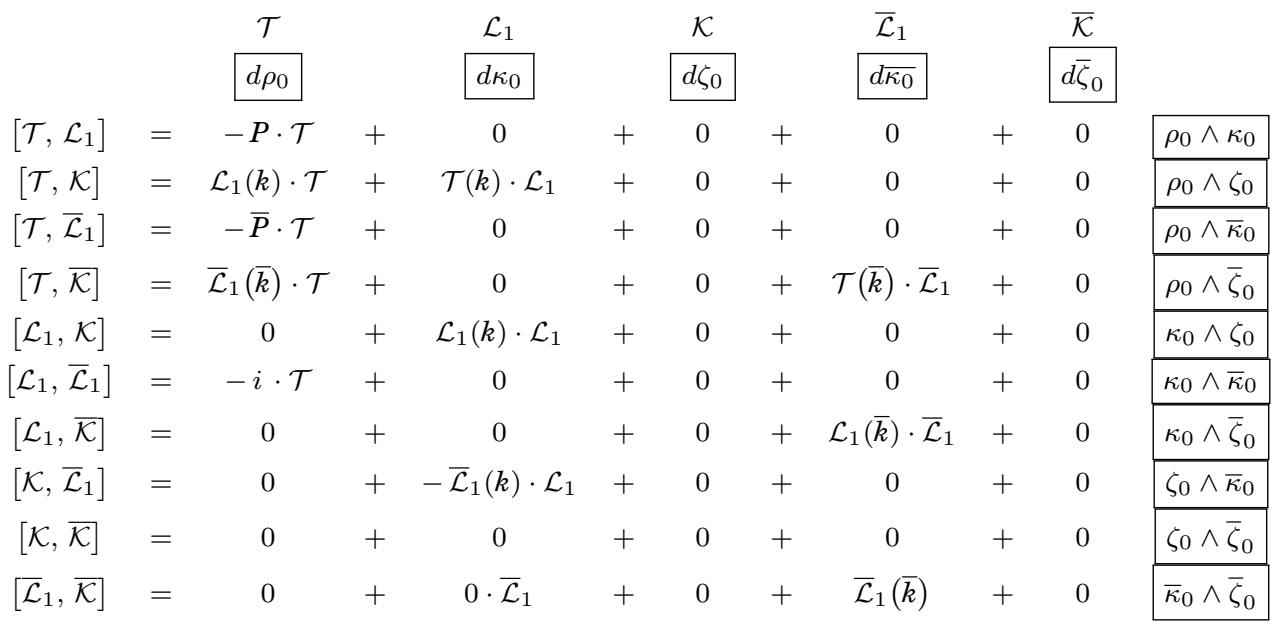

and by reading this array vertically, we obtain the initial Darboux-Cartan structure:

$$
\begin{aligned}
& d \rho_{0}=P \cdot \rho_{0} \wedge \kappa_{0}-\mathcal{L}_{1}(k) \cdot \rho_{0} \wedge \zeta_{0}+\bar{P} \cdot \rho_{0} \wedge \bar{\kappa}_{0}-\overline{\mathcal{L}}_{1}(\bar{k}) \cdot \rho_{0} \wedge \bar{\zeta}_{0}+i \kappa_{0} \wedge \bar{\kappa}_{0}, \\
& d \kappa_{0}=-\mathcal{T}(k) \cdot \rho_{0} \wedge \zeta_{0}-\mathcal{L}_{1}(k) \cdot \kappa_{0} \wedge \zeta_{0}+\overline{\mathcal{L}}_{1}(k) \cdot \zeta_{0} \wedge \bar{\kappa}_{0}, \\
& d \zeta_{0}=0 \\
& d \bar{\kappa}_{0}=-\mathcal{T}(\bar{k}) \cdot \rho_{0} \wedge \bar{\zeta}_{0}-\mathcal{L}_{1}(\bar{k}) \cdot \kappa_{0} \wedge \bar{\zeta}_{0}-\overline{\mathcal{L}}_{1}(\bar{k}) \cdot \bar{\kappa}_{0} \wedge \bar{\zeta}_{0}, \\
& d \bar{\zeta}_{0}=0 .
\end{aligned}
$$

The fact that the frame $\left\{\mathcal{T}, \mathcal{L}_{1}, \mathcal{K}, \overline{\mathcal{L}}_{1}, \overline{\mathcal{K}}\right\}$ is dual to the coframe $\left\{\rho_{0}, \kappa_{0}, \zeta_{0}, \bar{\kappa}_{0}, \bar{\zeta}_{0}\right\}$ yields a formula that shall be used several times later.

Lemma 2.6. The exterior differential of any function $G=G\left(z_{1}, z_{2}, \bar{z}_{1}, \bar{z}_{2}, v\right)$ on $M$ expresses as

$$
d G=\mathcal{T}(G) \rho_{0}+\mathcal{L}_{1}(G) \kappa_{0}+\mathcal{K}(G) \zeta_{0}+\overline{\mathcal{L}}_{1}(G) \bar{\kappa}_{0}+\overline{\mathcal{K}}(G) \bar{\zeta}_{0}
$$

Proof. Indeed, starting from the definition

$$
d \boldsymbol{G}=\frac{\partial \boldsymbol{G}}{\partial v} d v+\frac{\partial \boldsymbol{G}}{\partial z_{1}} d z_{1}+\frac{\partial \boldsymbol{G}}{\partial z_{2}} d z_{2}+\frac{\partial \boldsymbol{G}}{\partial \bar{z}_{1}} d \bar{z}_{1}+\frac{\partial \boldsymbol{G}}{\partial \bar{z}_{2}} d \bar{z}_{2},
$$

and inverting the above coframe

$$
\begin{aligned}
d z_{2} & =\zeta_{0}, \\
d z_{1} & =\kappa_{0}+k \zeta_{0}, \\
d v & =\ell \rho_{0}+A^{1}\left(\kappa_{0}+k \zeta_{0}\right)+A^{2} \zeta_{0}+\bar{A}^{1}\left(\bar{\kappa}_{0}+\bar{k} \bar{\zeta}_{0}\right)+\bar{A}^{2} \bar{\zeta}_{0} \\
& =\ell \rho_{0}+A^{1} \kappa_{0}+\left(A^{2}+k A^{1}\right) \zeta_{0}+\text { conjugates }
\end{aligned}
$$

we can replace, reorganize - unwritting the redundant conjugates - and reach the formula

$$
\begin{aligned}
d G & \equiv \frac{\partial G}{\partial v}\left(\ell \rho_{0}+A^{1} \kappa_{0}+\left(A^{2}+k A^{1}\right) \zeta_{0}\right)+\frac{\partial G}{\partial z_{1}}\left(\kappa_{0}+k \zeta_{0}\right)+\frac{\partial G}{\partial z_{2}} \zeta_{0} \\
& \equiv\left(\ell \frac{\partial}{\partial v}\right)(G) \cdot \rho_{0}+\left(\frac{\partial}{\partial z_{1}}+A^{1} \frac{\partial}{\partial v}\right)(G) \cdot \kappa_{0}+\left(\frac{\partial}{\partial z_{1}}+A^{2} \frac{\partial}{\partial v}+k \frac{\partial}{\partial z_{2}}+k A^{1} \frac{\partial}{\partial v}\right)(G) \cdot \zeta_{0} .
\end{aligned}
$$


For later much deeper computations, we need strong notational conventions. The order succession for our five 1 -forms which we will constantly use

$$
\left\{\rho_{0}, \kappa_{0}, \zeta_{0}, \bar{\kappa}_{0}, \bar{\zeta}_{0},\right\}
$$

induces an order succession for the ten generated 2-forms on the 5-dimensional CR manifold $M$

$$
\begin{aligned}
& \rho_{0} \wedge \kappa_{0} \quad \rho_{0} \wedge \zeta_{\mathbf{2}} \quad \rho_{0} \wedge \bar{\kappa}_{0} \quad \rho_{0} \wedge \bar{\zeta}_{0} \\
& \kappa_{0} \wedge_{\mathbf{5}} \zeta_{0} \quad \kappa_{0}{\underset{\mathbf{6}}{\mathbf{6}}}_{\bar{\kappa}_{0}} \quad \kappa_{0} \wedge \bar{\zeta}_{0} \\
& \zeta_{0} \wedge_{8} \bar{\kappa}_{0} \quad \zeta_{0} \wedge \bar{\zeta}_{0} \\
& \bar{\kappa}_{0} \wedge \bar{\zeta}_{0} \\
& 10
\end{aligned}
$$

With such a numbering, we can abreviate the structure equations as — dropping their conjugates -

$$
\begin{aligned}
d \rho_{0} & =R_{0}^{1} \rho_{0} \wedge \kappa_{0}+R_{0}^{2} \rho_{0} \wedge \zeta_{0}+R_{0}^{3} \rho_{0} \wedge \bar{\kappa}_{0}+R_{0}^{4} \rho_{0} \wedge \bar{\zeta}_{0}+i \kappa_{0} \wedge \bar{\kappa}_{0}, \\
d \kappa_{0} & =K_{0}^{2} \rho_{0} \wedge \zeta_{0}+K_{0}^{5} \kappa_{0} \wedge \zeta_{0}+K_{0}^{8} \zeta_{0} \wedge \bar{\kappa}_{0}, \\
d \zeta_{0} & =0 .
\end{aligned}
$$

Convention 2.7. All functions of $p=\left(z_{1}, z_{2}, \bar{z}_{1}, \bar{z}_{2}, v\right) \in M$ will be denoted with a lower index $(\bullet)_{0}$, always employing the special auxiliary font characters $A, B, C, \ldots$

After some transformations in the next sections, this initial coframe will change and become more complicated (unwriting the conjugates)

$$
\left\{\rho_{0}, \kappa_{0}, \zeta_{0}\right\} \quad \rightsquigarrow\left\{\rho_{0}, \kappa_{0}, \zeta_{0}^{\prime}\right\} \quad \rightsquigarrow\left\{\rho_{0}, \kappa_{0}^{\prime}, \zeta_{0}^{\prime}\right\} \rightsquigarrow\left\{\rho_{0}, \kappa_{0}^{\prime}, \zeta_{0}^{\prime \prime}\right\},
$$

and new structure function $R_{0}^{i \prime}, K_{0}^{i \prime}, Z_{0}^{i \prime}, \ldots$ will appear.

We end up this section by stating some technical commutation relations that shall be constantly necessary to normalize incoming (complicated) expressions in order to avoid ambiguities. In fact, we can take advantage of $\overline{\mathcal{K}}(k)=0$ from Lemma 2.5 , to make $\overline{\mathcal{K}}$ 'jump' above iterated derivatives like $e . g$. in
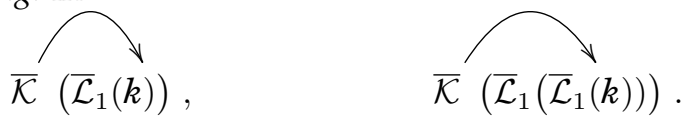

Precisely, the last, $10^{\text {th }}$ Lie bracket relation preceding Lemma 2.5

$$
-\overline{\mathcal{L}}_{1}(\bar{k}) \cdot \overline{\mathcal{L}}_{1}(\bullet)=\left[\overline{\mathcal{K}}, \overline{\mathcal{L}}_{1}\right](\bullet)
$$

when applied to the function $\bullet:=k$ yields

$$
\begin{aligned}
-\overline{\mathcal{L}}_{1}(\bar{k}) \overline{\mathcal{L}}_{1}(k)=\left[\overline{\mathcal{K}}, \overline{\mathcal{L}}_{1}\right](k) & =\overline{\mathcal{K}}\left(\overline{\mathcal{L}}_{1}(k)\right)-\overline{\mathcal{L}}_{1}(\overline{\mathcal{K}}(k) \\
& =\overline{\mathcal{K}}\left(\overline{\mathcal{L}}_{1}(k)\right) .
\end{aligned}
$$

Lemma 2.9. One has the 3 relations

$$
\begin{aligned}
\overline{\mathcal{K}}\left(\overline{\mathcal{L}}_{1}(k)\right)= & -\overline{\mathcal{L}}_{1}(\bar{k}) \overline{\mathcal{L}}_{1}(k) \\
\overline{\mathcal{K}}\left(\overline{\mathcal{L}}_{1}\left(\overline{\mathcal{L}}_{1}(k)\right)\right)= & -2 \overline{\mathcal{L}}_{1}(\bar{k}) \overline{\mathcal{L}}_{1}\left(\overline{\mathcal{L}}_{1}(k)\right)-\overline{\mathcal{L}}_{1}\left(\overline{\mathcal{L}}_{1}(\bar{k})\right) \overline{\mathcal{L}}_{1}(k), \\
\overline{\mathcal{K}}\left(\overline{\mathcal{L}}_{1}\left(\overline{\mathcal{L}}_{1}\left(\overline{\mathcal{L}}_{1}(k)\right)\right)\right)= & -3 \overline{\mathcal{L}}_{1}(\bar{k}) \overline{\mathcal{L}}_{1}\left(\overline{\mathcal{L}}_{1}\left(\overline{\mathcal{L}}_{1}(k)\right)\right) \\
& -3 \overline{\mathcal{L}}_{1}\left(\overline{\mathcal{L}}_{1}(\bar{k})\right) \overline{\mathcal{L}}_{1}\left(\overline{\mathcal{L}}_{1}(k)\right)-\overline{\mathcal{L}}_{1}\left(\overline{\mathcal{L}}_{1}\left(\overline{\mathcal{L}}_{1}(\bar{k})\right)\right) \overline{\mathcal{L}}_{1}(k) .
\end{aligned}
$$


Proof. As (1) is done, we can apply $\overline{\mathcal{L}}_{1}(\bullet)$ to it, reversing sides

$$
-\overline{\mathcal{L}}_{1}\left(\overline{\mathcal{L}}_{1}(\bar{k})\right) \overline{\mathcal{L}}_{1}(k)-\overline{\mathcal{L}}_{1}(\bar{k}) \overline{\mathcal{L}}_{1}\left(\overline{\mathcal{L}}_{1}(k)\right)=\overline{\mathcal{L}}_{1}\left(\overline{\mathcal{K}}\left(\overline{\mathcal{L}}_{1}(k)\right)\right) .
$$

Similarly, we apply $(2.8)$ to $\bullet:=\overline{\mathcal{L}}_{1}(k)$ and we reach (2) after a replacement

$$
-\overline{\mathcal{L}}_{1}(\bar{k}) \overline{\mathcal{L}}_{1}\left(\overline{\mathcal{L}}_{1}(k)\right)=\left[\overline{\mathcal{K}}, \overline{\mathcal{L}}_{1}\right]\left(\overline{\mathcal{L}}_{1}(k)\right)=\overline{\mathcal{K}}\left(\overline{\mathcal{L}}_{1}\left(\overline{\mathcal{L}}_{1}(k)\right)\right)-\underbrace{\overline{\mathcal{L}}_{1}\left(\overline{\mathcal{K}}\left(\overline{\mathcal{L}}_{1}(k)\right)\right)}_{\text {replace }} .
$$

Now, as (2) is done, we can apply $\overline{\mathcal{L}}_{1}(\bullet)$ to it, and get after reorganization

$$
\begin{aligned}
\overline{\mathcal{L}}_{1}\left(\overline{\mathcal{K}}\left(\overline{\mathcal{L}}_{1}\left(\overline{\mathcal{L}}_{1}(k)\right)\right)\right)= & -2 \overline{\mathcal{L}}_{1}(\bar{k}) \overline{\mathcal{L}}_{1}\left(\overline{\mathcal{L}}_{1}\left(\overline{\mathcal{L}}_{1}(k)\right)\right)-3 \overline{\mathcal{L}}_{1}\left(\overline{\mathcal{L}}_{1}(\bar{k})\right) \overline{\mathcal{L}}_{1}\left(\overline{\mathcal{L}}_{1}(k)\right) \\
& -\overline{\mathcal{L}}_{1}\left(\overline{\mathcal{L}}_{1}\left(\overline{\mathcal{L}}_{1}(\bar{k})\right)\right) \overline{\mathcal{L}}_{1}(k) .
\end{aligned}
$$

Lastly, we apply (2.8) to $\bullet:=\overline{\mathcal{L}}_{1}\left(\overline{\mathcal{L}}_{1}(k)\right)$ and we reach (3) after a replacement

$$
\begin{aligned}
-\overline{\mathcal{L}}_{1}(\bar{k}) \overline{\mathcal{L}}_{1}\left(\overline{\mathcal{L}}_{1}\left(\overline{\mathcal{L}}_{1}(k)\right)\right) & =\left[\overline{\mathcal{K}}, \overline{\mathcal{L}}_{1}\right]\left(\overline{\mathcal{L}}_{1}\left(\overline{\mathcal{L}}_{1}(k)\right)\right) \\
& =\overline{\mathcal{K}}\left(\overline{\mathcal{L}}_{1}\left(\overline{\mathcal{L}}_{1}\left(\overline{\mathcal{L}}_{1}(k)\right)\right)\right)-\underbrace{\overline{\mathcal{L}}_{1}\left(\overline{\mathcal{K}}\left(\overline{\mathcal{L}}_{1}\left(\overline{\mathcal{L}}_{1}(k)\right)\right)\right)}_{\text {replace }} .
\end{aligned}
$$

3. INITIAL $G_{1}$-STRUCTURE FOR LOCAL BIHOLOMORPHIC EQUIVALENCES $h: M \stackrel{\sim}{\longrightarrow} M^{\prime}$

Now, let $h: U \stackrel{\sim}{\longrightarrow} U^{\prime} \subset \mathbb{C}^{3}$ be a (local) biholomorphism from an open set $U \subset \mathbb{C}^{3}$ containing $U \ni 0$ the origin onto its image

$$
h(U)=: U^{\prime} \ni 0^{\prime}=h(0),
$$

which is also an open set $U^{\prime} \subset \mathbb{C}^{\prime 3}$ containing the origin $0^{\prime}$ in another target complex Euclidean space $\mathbb{C}^{\prime 3}$ having the same dimension.
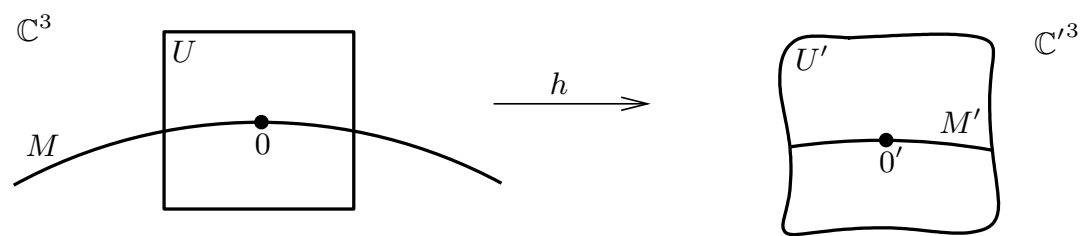

As in Cartan's equivalence theory, assume that $h(M \cap U) \subset M^{\prime}$ is contained in another real hypersurface $M^{\prime} \subset \mathbb{C}^{\prime 3}$, also passing through the origin $0^{\prime} \in M^{\prime}$, represented in holomorphic coordinates $\left(z_{1}^{\prime}, z_{2}^{\prime}, w^{\prime}=u^{\prime}+i v^{\prime}\right)$ by a similar $\mathcal{C}^{\omega}$ graphed equation

$$
u^{\prime}=F^{\prime}\left(z_{1}^{\prime}, z_{2}^{\prime}, \bar{z}_{1}^{\prime}, \bar{z}_{2}^{\prime}, v^{\prime}\right) .
$$

We now make the convention of not mentioning the open sets that must sometimes be shrunk, so that we think of $h: M \stackrel{\sim}{\longrightarrow} M^{\prime}$ as being a $\mathrm{CR}$ equivalence between hypersurfaces $M \subset \mathbb{C}^{3}$ and $M^{\prime} \subset \mathbb{C}^{\prime 3}$.

In the target space, introduce similar generators $\mathcal{L}_{1}^{\prime}, \mathcal{L}_{2}^{\prime}$ for $T^{1,0} M^{\prime}$. Since $h$ is holomorphic, its differential $h_{*}: \mathbb{C} T \mathbb{C}^{3} \longrightarrow \mathbb{C} T \mathbb{C}^{3}$ stabilizes holomorphic $(1,0)$ and holomorphic $(0,1)$ vector fields

$$
h_{*}\left(T^{1,0} \mathbb{C}^{3}\right)=T^{1,0} \mathbb{C}^{\prime 3} \quad \text { and } \quad h_{*}\left(T^{0,1} M\right)=T^{0,1} M^{\prime} .
$$

Furthermore, by invariancy of the Freeman form, $h$ respects the Levi-kernel distributions

$$
h_{*}\left(K^{1,0} M\right)=K^{1,0} M^{\prime} .
$$


Consequently, there exist functions $f^{\prime}, c^{\prime}, e^{\prime}$ on $M^{\prime}$ such that

$$
\begin{aligned}
h_{*}(\mathcal{K}) & =f^{\prime} \mathcal{K}^{\prime}, \\
h_{*}\left(\mathcal{L}_{1}\right) & =c^{\prime} \mathcal{L}_{1}^{\prime}+e^{\prime} \mathcal{K}^{\prime},
\end{aligned}
$$

whence by conjugation

$$
\begin{aligned}
h_{*}(\overline{\mathcal{K}}) & =\bar{f}^{\prime} \overline{\mathcal{K}}^{\prime}, \\
h_{*}\left(\overline{\mathcal{L}}_{1}\right) & =\bar{c}^{\prime} \overline{\mathcal{L}}_{1}^{\prime}+\bar{e}^{\prime} \overline{\mathcal{K}}^{\prime} .
\end{aligned}
$$

On the other hand, there is a priori no special condition that shall be satisfied by $h_{*}(\mathcal{T})$, except that it be a real vector field, because $\mathcal{T}$ is real. Thus, there are a real-valued function $a^{\prime}$ and two complex-valued $b^{\prime}$ and $d^{\prime}$ on $M^{\prime}$ such that

$$
h_{*}(\mathcal{T})=a^{\prime} \mathcal{T}^{\prime}+b^{\prime} \mathcal{L}_{1}^{\prime}+d^{\prime} \mathcal{K}^{\prime}+\bar{b}^{\prime} \overline{\mathcal{L}}_{1}^{\prime}+\bar{d}^{\prime} \overline{\mathcal{K}}^{\prime} .
$$

In fact, the function $a^{\prime}$ is determined, because

$$
\begin{aligned}
h_{*}(\mathcal{T})=h_{*}\left(i\left[\mathcal{L}_{1}, \overline{\mathcal{L}}_{1}\right]\right) & =i\left[h_{*}\left(\mathcal{L}_{1}\right), h_{*}\left(\overline{\mathcal{L}}_{1}\right)\right] \\
& =i\left[c^{\prime} \mathcal{L}_{1}^{\prime}+e^{\prime} \mathcal{K}^{\prime}, \bar{c}^{\prime} \overline{\mathcal{L}}_{1}^{\prime}+\bar{e}^{\prime} \overline{\mathcal{K}}^{\prime}\right] \\
& \equiv c^{\prime} \bar{c}^{\prime} i\left[\mathcal{L}_{1}^{\prime}, \overline{\mathcal{L}}_{1}^{\prime}\right] \quad \bmod \left(T^{1,0} M^{\prime} \oplus T^{0,1} M^{\prime}\right),
\end{aligned}
$$

whence necessarily

$$
a^{\prime}=c^{\prime} \bar{c}^{\prime} .
$$

Summarizing, we have the following matrix relations

$$
h_{*}\left(\begin{array}{c}
\mathcal{T} \\
\mathcal{L}_{1} \\
\mathcal{K} \\
\overline{\mathcal{L}}_{1} \\
\overline{\mathcal{K}}
\end{array}\right)=\left(\begin{array}{ccccc}
c^{\prime} \bar{c}^{\prime} & b^{\prime} & d^{\prime} & \bar{b}^{\prime} & \bar{d}^{\prime} \\
0 & c^{\prime} & e^{\prime} & 0 & 0 \\
0 & 0 & f^{\prime} & 0 & 0 \\
0 & 0 & 0 & \bar{c}^{\prime} & \bar{e}^{\prime} \\
0 & 0 & 0 & 0 & \bar{f}^{\prime}
\end{array}\right)\left(\begin{array}{c}
\mathcal{T}^{\prime} \\
\mathcal{L}_{1}^{\prime} \\
\mathcal{K}^{\prime} \\
\overline{\mathcal{L}}_{1}^{\prime} \\
\overline{\mathcal{K}}^{\prime}
\end{array}\right) .
$$

As $h_{*}$ is invertible, the function $f^{\prime}$, and then the function $c^{\prime}$ too, must be nowhere vanishing. The relation between the coframe $\left\{\rho_{0}, \kappa_{0}, \zeta_{0}, \bar{\kappa}_{0}, \bar{\zeta}_{0}\right\}$ in the source space and the coframe $\left\{\rho_{0}^{\prime}, \kappa_{0}^{\prime}, \zeta_{0}^{\prime}, \bar{\kappa}_{0}^{\prime}, \bar{\zeta}_{0}^{\prime}\right\}$ in the target space is therefore given by a plain transposition

$$
h^{*}\left(\begin{array}{c}
\rho_{0}^{\prime} \\
\kappa_{0}^{\prime} \\
\zeta_{0}^{\prime} \\
\bar{\kappa}_{0}^{\prime} \\
\bar{\zeta}_{0}^{\prime}
\end{array}\right)=\left(\begin{array}{ccccc}
c^{\prime} \bar{c}^{\prime} & 0 & 0 & 0 & 0 \\
b^{\prime} & c^{\prime} & 0 & 0 & 0 \\
d^{\prime} & e^{\prime} & f^{\prime} & 0 & 0 \\
\bar{b}^{\prime} & 0 & 0 & \bar{c}^{\prime} & 0 \\
\bar{d}^{\prime} & 0 & 0 & \bar{e}^{\prime} & \bar{f}^{\prime}
\end{array}\right)\left(\begin{array}{c}
\rho_{0} \\
\kappa_{0} \\
\zeta_{0} \\
\bar{\kappa}_{0} \\
\bar{\zeta}_{0}
\end{array}\right) .
$$

These preliminaries, also explained in $[16,25,18]$, justify that the initial $G$-structure for such equivalences of CR manifolds is the matrix ambiguity group $G_{1}$ is constituted of $5 \times 5$ matrices of the form

$$
\left(\begin{array}{ccccc}
c \bar{c} & 0 & 0 & 0 & 0 \\
b & c & 0 & 0 & 0 \\
d & e & f & 0 & 0 \\
\bar{b} & 0 & 0 & \bar{c} & 0 \\
\bar{d} & 0 & 0 & \bar{e} & \bar{f}
\end{array}\right)
$$

with free variable complex entries

$$
\text { c, } f \in \mathbb{C} \backslash\{0\} \quad \text { and } \quad \text { b, d, e } \in \mathbb{C} \text {, }
$$


namely

$$
\left(\begin{array}{c}
\rho \\
\kappa \\
\zeta \\
\bar{\kappa} \\
\bar{\zeta}
\end{array}\right):=\left(\begin{array}{ccccc}
\mathrm{c} \overline{\mathrm{c}} & 0 & 0 & 0 & 0 \\
\mathrm{~b} & \mathrm{c} & 0 & 0 & 0 \\
\mathrm{~d} & \mathrm{e} & \mathrm{f} & 0 & 0 \\
\overline{\mathrm{b}} & 0 & 0 & \overline{\mathrm{c}} & 0 \\
\overline{\mathrm{d}} & 0 & 0 & \overline{\mathrm{e}} & \overline{\mathrm{f}}
\end{array}\right)\left(\begin{array}{c}
\rho_{0} \\
\kappa_{0} \\
\zeta_{0} \\
\bar{\kappa}_{0} \\
\bar{\zeta}_{0}
\end{array}\right) .
$$

Eliminating the conjugate 1 -forms $\bar{\kappa}, \bar{\zeta}$ for which the structure equations are redundant, this can be abbreviated as

$$
\left(\begin{array}{c}
\rho \\
\kappa \\
\zeta
\end{array}\right):=\left(\begin{array}{ccc}
c \bar{c} & 0 & 0 \\
\mathrm{~b} & \mathrm{c} & 0 \\
\mathrm{~d} & \mathrm{e} & \mathrm{f}
\end{array}\right)\left(\begin{array}{c}
\rho_{0} \\
\kappa_{0} \\
\zeta_{0}
\end{array}\right) .
$$

\section{A Labyrinthmap to PocChiOla's CALCUlations}

The successive reductions of this $G_{1}$ structure will look as

$$
\begin{aligned}
g:=\left(\begin{array}{ccc}
\mathrm{c} \overline{\mathrm{c}} & 0 & 0 \\
\mathrm{~b} & \mathrm{c} & 0 \\
\mathrm{~d} & \mathrm{e} & \mathrm{f}
\end{array}\right) \rightsquigarrow \quad g:=\left(\begin{array}{ccc}
\mathrm{c} \overline{\mathrm{c}} & 0 & 0 \\
\mathrm{~b} & \mathrm{c} & 0 \\
\mathrm{~d} & \mathrm{e} & \overline{\mathrm{c}}
\end{array}\right) & \rightsquigarrow \quad g:=\left(\begin{array}{ccc}
\mathrm{c} \overline{\mathrm{c}} & 0 & 0 \\
-i \overline{\mathrm{c}} \mathrm{e} & \mathrm{c} & 0 \\
\mathrm{~d} & \mathrm{e} & \frac{\mathrm{c}}{\overline{\mathrm{c}}}
\end{array}\right) \\
& \rightsquigarrow \quad g:=\left(\begin{array}{cccc}
\mathrm{c} \overline{\mathrm{c}} & 0 & 0 \\
-i \overline{\mathrm{c} e} & \mathrm{c} & 0 \\
-\frac{i}{2} \frac{\overline{\mathrm{c}} e^{2}}{\mathrm{c}} & \mathrm{e} & \overline{\mathrm{c}}
\end{array}\right),
\end{aligned}
$$

thanks to successive normalization of some group parameters (offered by some essential torsion coefficients yielding invariants that are deeper than Levi and Freeman forms)

$$
\mathrm{f}:=\frac{\mathrm{c}}{\overline{\mathrm{c}}} \overline{\mathcal{L}}_{1}(k), \quad \mathrm{b}:=-i \overline{\mathrm{c}} \mathrm{e}+\frac{i}{3} \mathrm{c} B_{0}, \quad \mathrm{~d}:=-\frac{i}{2} \frac{\overline{\mathrm{c} e \mathrm{e}}}{\mathrm{c}}+i \frac{\mathrm{c}}{\overline{\mathrm{c}}} H_{0},
$$

in terms of the following two function on $M$

$$
\begin{aligned}
B_{0} & :=\frac{\overline{\mathcal{L}}_{1}\left(\overline{\mathcal{L}}_{1}(k)\right)}{\overline{\mathcal{L}}_{1}(k)}-\bar{P} \\
H_{0} & :=-\frac{1}{6} \frac{\overline{\mathcal{L}}_{1}\left(\overline{\mathcal{L}}_{1}\left(\overline{\mathcal{L}}_{1}(k)\right)\right)}{\overline{\mathcal{L}}_{1}(k)}+\frac{2}{9} \frac{\overline{\mathcal{L}}_{1}\left(\overline{\mathcal{L}}_{1}(k)\right)^{2}}{\overline{\mathcal{L}}_{1}(k)^{2}}+\frac{1}{18} \frac{\overline{\mathcal{L}}_{1}\left(\overline{\mathcal{L}}_{1}(k)\right) \bar{P}}{\overline{\mathcal{L}}_{1}(k)}+\frac{1}{6} \overline{\mathcal{L}_{1}}(\bar{P})-\frac{1}{9} \bar{P}^{2} .
\end{aligned}
$$

This function $H_{0}$ coincides with Pocchiola's function $H$.

The next sections will present in details these successive reductions of $G$-structures, by these normalizations of the group parameters $f, b, d$. Contrary to [18, 25], all computations will be progressive, simple, detailed, readable, clear, without needing any help of either a computer or a pen. A great care will be devoted to readability.

\section{First LoOP: REDUCtion OF THE GROUP PARAMETER $f$}

We recall that the initial Darboux-Cartan structure of the coframe $\left\{\rho_{0}, \kappa_{0}, \zeta_{0}, \bar{\kappa}_{0}, \bar{\zeta}_{0}\right\}$ is, without writing conjugate equations - remind $\bar{\rho}_{0}=\rho_{0}-$

$$
\begin{aligned}
d \rho_{0} & =P \rho_{0} \wedge \kappa_{0}-\mathcal{L}_{1}(k) \rho_{0} \wedge \zeta_{0}+\bar{P} \rho_{0} \wedge \bar{\kappa}_{0}-\overline{\mathcal{L}}_{1}(\bar{k}) \rho_{0} \wedge \bar{\zeta}_{0}+i \kappa_{0} \wedge \bar{\kappa}_{0}, \\
d \kappa_{0} & =-\mathcal{T}(k) \rho_{0} \wedge \zeta_{0}-\mathcal{L}_{1}(k) \kappa_{0} \wedge \zeta_{0}+\overline{\mathcal{L}}_{1}(k) \zeta_{0} \wedge \bar{\kappa}_{0}, \\
d \zeta_{0} & =0 .
\end{aligned}
$$


With the first $G$-structure exhibited above, introduce the lifted differential forms, defined by

$$
\left(\begin{array}{l}
\rho \\
\kappa \\
\zeta
\end{array}\right):=\left(\begin{array}{ccc}
c \bar{c} & 0 & 0 \\
b & c & 0 \\
d & e & f
\end{array}\right)\left(\begin{array}{c}
\rho_{0} \\
\kappa_{0} \\
\zeta_{0}
\end{array}\right),
$$

id est

$$
\begin{aligned}
\rho & :=\mathrm{c} \overline{\mathrm{c}} \rho_{0}, \\
\kappa & :=\mathrm{b} \rho_{0}+\mathrm{c} \kappa_{0}, \\
\zeta & :=\mathrm{d} \rho_{0}+\mathrm{e} \kappa_{0}+\mathrm{f} \zeta_{0} .
\end{aligned}
$$

Here, $c, f \in \mathbb{C}^{*}$ and $b, e, d \in \mathbb{C}$. Mind that conjugate equations giving $\bar{\kappa}$ and $\bar{\zeta}$ are not written, but will be used.

An inversion yields

$$
\begin{aligned}
\rho_{0} & =\frac{1}{\mathrm{c} \overline{\mathrm{c}}} \rho, \\
\kappa_{0} & =\frac{1}{\mathrm{c}} \kappa-\frac{\mathrm{b}}{\mathrm{ccc}} \rho, \\
\zeta_{0} & =\frac{\mathrm{be}-\mathrm{cd}}{\mathrm{cc} \overline{\mathrm{c} f}} \rho-\frac{\mathrm{e}}{\mathrm{cf}} \kappa+\frac{1}{\mathrm{f}} \zeta .
\end{aligned}
$$

With the above $3 \times 3$ matrix $g$ representing the general element of a 10-dimensional (real) group $G^{10} \subset \mathrm{GL}_{3}(\mathbb{C})$, the Maurer-Cartan matrix is

$$
\begin{aligned}
d g \cdot g^{-1} & =\left(\begin{array}{ccc}
\bar{c} d c+c d \bar{c} & 0 & 0 \\
d \mathrm{~b} & d \mathrm{c} & 0 \\
d \mathrm{~d} & d \mathrm{e} & d \mathrm{f}
\end{array}\right)\left(\begin{array}{ccc}
\frac{1}{\mathrm{c} \bar{b}} & 0 & 0 \\
-\frac{\mathrm{b}}{\mathrm{cc} \bar{c}} & \frac{1}{c} & 0 \\
\frac{\mathrm{c}-c \mathrm{c}}{\mathrm{cc} f} & -\frac{\mathrm{e}}{\mathrm{cf}} & \frac{1}{f}
\end{array}\right) \\
& =:\left(\begin{array}{ccc}
\alpha+\bar{\alpha} & 0 & 0 \\
\beta & \alpha & 0 \\
\gamma & \delta & \varepsilon
\end{array}\right)
\end{aligned}
$$

in terms of the group-invariant 1-forms

$$
\begin{aligned}
\alpha & :=\frac{d \mathrm{c}}{\mathrm{c}}, \\
\beta & :=\frac{d \mathrm{~b}}{\mathrm{c} \bar{c}}-\frac{\mathrm{b} \bar{c} d \mathrm{c}}{\mathrm{cc}}, \\
\gamma & :=\frac{d \mathrm{~d}}{\mathrm{c} \bar{c}}-\frac{\mathrm{b} d \mathrm{e}}{\mathrm{cc} \overline{\mathrm{c}}}+\frac{\mathrm{be}-\mathrm{cd}}{\mathrm{cc} \overline{\mathrm{c}}} d \mathrm{f}, \\
\delta & :=\frac{d \mathrm{e}}{\mathrm{c}}-\frac{\mathrm{e} d \mathrm{f}}{\mathrm{cf}}, \\
\varepsilon & :=\frac{d \mathrm{f}}{\mathrm{f}} .
\end{aligned}
$$

As is known, after painful computations whose outcomes are presented extensively in $[25,18]$, one can re-express, using (5.1) and (5.2), the exterior differentials of the 3 lifted 1-forms $\rho, \zeta, \kappa$ 
as

$$
\begin{aligned}
d \rho= & \alpha \wedge \rho+\bar{\alpha} \wedge \rho \\
& +R^{1} \rho \wedge \kappa+R^{2} \rho \wedge \zeta+R^{3} \rho \wedge \bar{\kappa}+R^{4} \rho \wedge \bar{\zeta}+i \kappa \wedge \bar{\kappa}, \\
d \kappa= & \beta \wedge \rho+\alpha \wedge \kappa \\
& +K^{1} \rho \wedge \kappa+K^{2} \rho \wedge \zeta+K^{3} \rho \wedge \bar{\kappa}+K^{4} \rho \wedge \bar{\zeta} \\
& +K^{5} \kappa \wedge \zeta+K^{5} \kappa \wedge \bar{\kappa}+K^{8} \zeta \wedge \bar{\kappa}, \\
d \zeta= & \gamma \wedge \rho+\delta \wedge \kappa+\varepsilon \wedge \zeta \\
& +Z^{1} \rho \wedge \kappa+Z^{2} \rho \wedge \zeta+Z^{3} \rho \wedge \bar{\kappa}+Z^{4} \rho \wedge \bar{\zeta} \\
& +Z^{5} \kappa \wedge \zeta+Z^{6} \kappa \wedge \bar{\kappa}+Z^{8} \zeta \wedge \bar{\kappa}
\end{aligned}
$$

in terms of certain complicated functions $R^{i}, K^{i}, Z^{i}$ of the horizontal variables and of the group parameters as well

$$
\left(z_{1}, z_{2}, \bar{z}_{1}, \bar{z}_{2}, v\right) \times(\mathrm{c}, \overline{\mathrm{c}}, \mathrm{f}, \overline{\mathrm{f}}, \mathrm{b}, \overline{\mathrm{b}}, \mathrm{d}, \overline{\mathrm{d}}, \mathrm{e}, \overline{\mathrm{e}}) \in M^{5} \times G^{10},
$$

but we shall not need the expressions of all these functions, and focus only on the boxed one, $K^{8}$, since it will bring an interesting normalization for the diagonal group parameter $\mathrm{f}$.

Notation 5.3. Given a differential 2-form $\Omega \in \Gamma\left(M, \Lambda^{2} T^{*} M\right)$ on an $n$-dimensional manifold $M$ equipped with a coframe $\left\{\omega^{1}, \ldots, \omega^{n}\right\}$ for its cotangent bundle $T^{*} M$, which is expanded as

$$
\Omega=\sum_{1 \leqslant i<j \leqslant n} A_{i, j} \omega^{i} \wedge \omega^{j},
$$

with uniquely determined coefficients-functions $A_{\bullet, \bullet}$, for fixed $i<j$, the coefficient $A_{i, j}$ of $\omega^{i} \wedge \omega^{j}$ will be denoted by

$$
\left[\omega^{i} \wedge \omega^{j}\right]\{\Omega\}:=A_{i, j} .
$$

To capture $K^{8}$ without pain, the computation/re-expression of $d \kappa$ starts from $\kappa=\mathrm{b} \rho_{0}+\mathrm{c} \kappa_{0}$ as follows to see how Maurer-Cartan forms enter the play

$$
\begin{aligned}
d \kappa & =d \mathrm{~b} \wedge \rho_{0}+d \mathrm{c} \wedge \kappa_{0}+\mathrm{b} d \rho_{0}+\mathrm{c} d \kappa_{0} \\
& =d \mathrm{~b} \wedge\left(\frac{1}{c \bar{c}} \rho\right)+d \mathrm{c} \wedge\left(\frac{1}{\mathrm{c}} \kappa-\frac{\mathrm{b}}{\mathrm{cc} \bar{c}} \rho\right)+\text { Torsion } \\
& =\left(d \mathrm{~b}-\frac{\mathrm{b} d \mathrm{c}}{\mathrm{cc} \bar{c}}\right) \wedge \rho+\left(\frac{d \mathrm{c}}{\mathrm{c}}\right) \wedge \kappa+\text { Torsion } \\
& =\beta \wedge \rho+\alpha \wedge \kappa+\text { Torsion. }
\end{aligned}
$$

Certainly, $K^{8}$ belongs to the torsion remainder, and we want to determine only

$$
K^{8}:=[\zeta \wedge \bar{\kappa}]\{d \kappa\}=[\zeta \wedge \bar{\kappa}]\left\{\mathrm{b} d \rho_{0}+\mathrm{c} d \kappa_{0}\right\} .
$$

For the first term $\mathrm{b} d \rho_{0}$, we look at (5.1) in which we replace visually $\rho_{0}, \zeta_{0}, \kappa_{0}$ by $\rho, \zeta, \kappa$ watching simultaneously (5.2) — no pen needed! computers shut down! — and we get

$$
\mathrm{b}[\zeta \wedge \bar{\kappa}]\left\{d \rho_{0}\right\}=0+0+0+0+0=0 .
$$

Proceeding similarly, just with eyes

$$
\begin{aligned}
\mathrm{c}[\zeta \wedge \bar{\kappa}]\left\{d \kappa_{0}\right\} & =0+0+\mathrm{c} \overline{\mathcal{L}}_{1}(k)[\zeta \wedge \bar{\kappa}]\left\{\left(\frac{\mathrm{be}-\mathrm{bd}}{\mathrm{cc} \overline{\mathrm{cf}}} \rho-\frac{\mathrm{e}}{\mathrm{cf}} \kappa+\frac{1}{\mathrm{f}} \zeta\right) \wedge\left(-\frac{\overline{\mathrm{b}}}{\overline{\mathrm{ccc}}} \rho+\frac{1}{\overline{\mathrm{c}}} \bar{\kappa}\right)\right. \\
& =\mathrm{c} \overline{\mathcal{L}}_{1}(k)\left(\frac{1}{\mathrm{f}}\right)\left(\frac{1}{\overline{\mathrm{c}}}\right),
\end{aligned}
$$

whence adding

$$
K^{8}=\frac{\mathrm{c}}{\overline{\mathrm{cf}}} \overline{\mathcal{L}}_{1}(k)
$$


Furthermore, without computation, we see that $K^{8}$ is not absorbable in the Maurer-Cartan part $\beta \wedge \rho+\alpha \wedge \kappa$ by means of any replacement

$$
\begin{aligned}
& \alpha=\alpha^{\prime}+a_{1} \rho+a_{2} \kappa+a_{3} \zeta+a_{4} \bar{\kappa}+a_{5} \bar{\zeta}, \\
& \beta=\beta^{\prime}+b_{1} \rho+b_{2} \kappa+b_{3} \zeta+b_{4} \bar{\kappa}+b_{5} \bar{\zeta},
\end{aligned}
$$

because the result will always be

$$
\text { something } \wedge \rho+\text { something } \wedge \kappa,
$$

whereas $K^{8} \zeta \wedge \bar{\kappa}$ is not $\wedge$-divisible by either $\wedge \rho$ or $\wedge \kappa$.

Consequently, $K^{8}$ is an essential torsion coefficient, and by general Cartan theory, $K^{8}$ may bring a group parameter normalization.

In fact, since the diagonal coefficients $c \neq 0 \neq \mathrm{f}$ of the invertible triangular matrix must be nonvanishing, and since $\overline{\mathcal{L}}_{1}(k) \neq 0$ is nowhere vanishing by our assumption of 2-nondegeneracy, it is natural, then, to normalize $K^{8}$ to be constant nonzero, e.g. $K^{8}:=1$, and this yields a reduction of the $G^{10}$-structure to an eight-dimensional $G^{8}$-structure by setting

$$
\mathrm{f}:=\frac{\mathrm{c}}{\overline{\mathrm{c}}} \overline{\mathcal{L}}_{1}(k) \text {. }
$$

Inserting this in the lifted coframe

$$
\left(\begin{array}{l}
\rho \\
\kappa \\
\zeta
\end{array}\right):=\left(\begin{array}{ccc}
\mathrm{c} \overline{\mathrm{c}} & 0 & 0 \\
\mathrm{~b} & \mathrm{c} & 0 \\
\mathrm{~d} & \mathrm{e} & \overline{\mathrm{c}} \\
\overline{\mathrm{c}} & \overline{\mathcal{L}}_{1}(k)
\end{array}\right)\left(\begin{array}{c}
\rho_{0} \\
\kappa_{0} \\
\zeta_{0}
\end{array}\right),
$$

we are conducted to change the initial coframe by introducing the new horizontal - i.e. defined on $M-1$-form

$$
\zeta_{0}^{\prime}:=\overline{\mathcal{L}}_{1}(k) \zeta_{0}
$$

As anticipated in a summary supra, we are thus changing of horizontal coframe

$$
\left\{\rho_{0}, \kappa_{0}, \zeta_{0}, \bar{\kappa}_{0}, \bar{\zeta}_{0}\right\} \quad \rightsquigarrow \quad\left\{\rho_{0}, \kappa_{0}, \zeta_{0}^{\prime}, \bar{\kappa}_{0}, \bar{\zeta}_{0}^{\prime}\right\},
$$

and unavoidably, we have to set up its Darboux-Cartan structure.

Thanks to Lemma 2.6, we can compute

$$
\begin{aligned}
d \zeta_{0}^{\prime}=d\left(\overline{\mathcal{L}}_{1}(k)\right) \wedge \zeta_{0}+\overline{\mathcal{L}}_{1}(k) \wedge \underline{d \zeta_{0}} & \\
=\mathcal{T}\left(\overline{\mathcal{L}}_{1}(k)\right) \rho_{0} \wedge \zeta_{0}+\mathcal{L}_{1}\left(\overline{\mathcal{L}}_{1}(k)\right) \kappa_{0} \wedge \zeta_{0} & +\mathcal{K}\left(\overline{\mathcal{L}}_{1}(k)\right) \underline{\zeta_{0} \wedge \zeta_{0}}+\overline{\mathcal{L}}_{1}\left(\overline{\mathcal{L}}_{1}(k)\right) \bar{\kappa}_{0} \wedge \zeta_{0} \\
& +\overline{\mathcal{K}}\left(\overline{\mathcal{L}}_{1}(k)\right) \bar{\zeta}_{0} \wedge \zeta_{0}+0,
\end{aligned}
$$

and next, replacing everywhere $\zeta_{0}=\frac{\zeta_{0}^{\prime}}{\overline{\mathcal{L}_{1}(k)}}$, reorganizing, and transforming the last term above in application of Lemma 2.9 (1), we obtain the structure equations enjoyed by this new initial base coframe

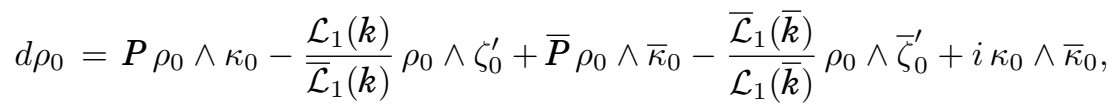

$$
\begin{aligned}
d \kappa_{0} & =-\frac{\mathcal{T}(k)}{\overline{\mathcal{L}}_{1}(k)} \rho_{0} \wedge \zeta_{0}^{\prime}-\frac{\mathcal{L}_{1}(k)}{\overline{\mathcal{L}}_{1}(k)} \kappa_{0} \wedge \zeta_{0}^{\prime}+\zeta_{0}^{\prime} \wedge \bar{\kappa}_{0}, \\
d \zeta_{0}^{\prime} & =\frac{\mathcal{T}\left(\overline{\mathcal{L}}_{1}(k)\right)}{\overline{\mathcal{L}}_{1}(k)} \rho_{0} \wedge \zeta_{0}^{\prime}+\frac{\mathcal{L}_{1}\left(\overline{\mathcal{L}}_{1}(k)\right)}{\overline{\mathcal{L}}_{1}(k)} \kappa_{0} \wedge \zeta_{0}^{\prime}-\frac{\overline{\mathcal{L}}_{1}\left(\overline{\mathcal{L}}_{1}(k)\right)}{\overline{\mathcal{L}}_{1}(k)} \zeta_{0}^{\prime} \wedge \bar{\kappa}_{0}+\frac{\overline{\mathcal{L}}_{1}(\bar{k})}{\mathcal{L}_{1}(\bar{k})} \zeta_{0}^{\prime} \wedge \bar{\zeta}_{0}^{\prime} .
\end{aligned}
$$


Sometimes, it can be useful to abbreviate these formulas as

$$
\begin{aligned}
& d \rho_{0}=R_{0}^{1} \rho_{0} \wedge \kappa_{0}+R_{0}^{2} \rho_{0} \wedge \zeta_{0}^{\prime}+\bar{R}_{0}^{1} \rho_{0} \wedge \bar{\kappa}_{0}+\bar{R}_{2}^{0} \rho_{0} \wedge \bar{\zeta}_{0}^{\prime}+i \kappa_{0} \wedge \bar{\kappa}_{0}, \\
& d \kappa_{0}=K_{0}^{2} \rho_{0} \wedge \zeta_{0}^{\prime}+K_{0}^{5} \kappa_{0} \wedge \zeta_{0}^{\prime}+\zeta_{0}^{\prime} \wedge \bar{\kappa}_{0}, \\
& d \zeta_{0}^{\prime}=Z_{0}^{2} \rho_{0} \wedge \zeta_{0}^{\prime}+Z_{0}^{5} \kappa_{0} \wedge \zeta_{0}^{\prime}+Z_{0}^{8} \zeta_{0}^{\prime} \wedge \bar{\kappa}_{0}+Z_{0}^{9} \zeta_{0}^{\prime} \wedge \bar{\zeta}_{0}^{\prime},
\end{aligned}
$$

and no primes will be appended to these coefficients-functions, for the reason that exactly two further changes of initial base coframes

$$
\left\{\rho_{0}, \kappa_{0}, \zeta_{0}^{\prime}, \bar{\kappa}_{0}, \bar{\zeta}_{0}^{\prime}\right\} \quad \rightsquigarrow\left\{\rho_{0}, \kappa_{0}^{\prime}, \zeta_{0}^{\prime}, \bar{\kappa}_{0}^{\prime}, \bar{\zeta}_{0}^{\prime}\right\} \rightsquigarrow\left\{\rho_{0}, \kappa_{0}^{\prime}, \zeta_{0}^{\prime \prime}, \bar{\kappa}_{0}^{\prime}, \bar{\zeta}_{0}^{\prime \prime}\right\}
$$

will force us to introduce e.g. $Z_{0}^{i \prime}$ and $Z_{0}^{i \prime \prime}$, so that we will avoid to use primes trice.

\section{SECOND LOOP: Reduction OF THE GROUP PARAMETER b}

With this new reduced (real) eight-dimensional group $G^{8}$, the lifted coframe, in which for simplicity, we use the same letters $\rho, \kappa, \zeta$ as before, becomes

$$
\left(\begin{array}{l}
\rho \\
\kappa \\
\zeta
\end{array}\right):=\left(\begin{array}{ccc}
\mathrm{c} \overline{\mathrm{c}} & 0 & 0 \\
\mathrm{~b} & \mathrm{c} & 0 \\
\mathrm{~d} & \mathrm{e} & \mathrm{c}
\end{array}\right)\left(\begin{array}{c}
\rho_{0} \\
\kappa_{0} \\
\zeta_{0}^{\prime}
\end{array}\right) \quad \Longleftrightarrow \quad\left\{\begin{array}{l}
\rho:=\mathrm{c} \overline{\mathrm{c}} \rho_{0}, \\
\kappa:=\mathrm{b} \rho_{0}+\mathrm{c} \kappa_{0}, \\
\zeta:=\mathrm{d} \rho_{0}+\mathrm{e} \kappa_{0}+\frac{\mathrm{c}}{\overline{\mathrm{c}}} \zeta_{0}^{\prime}
\end{array}\right.
$$

and inverse formulas are

$$
\begin{aligned}
\rho_{0} & =\frac{1}{\mathrm{c} \overline{\mathrm{c}}} \rho, \\
\kappa_{0} & =-\frac{\mathrm{b}}{\mathrm{cc} \overline{\mathrm{c}}} \rho+\frac{1}{\mathrm{c}} \kappa, \\
\zeta_{0}^{\prime} & =\frac{\mathrm{be}-\mathrm{cd}}{\mathrm{ccc}} \rho-\frac{\overline{\mathrm{ce}}}{\mathrm{cc}} \kappa+\frac{\overline{\mathrm{c}}}{\mathrm{c}} \zeta .
\end{aligned}
$$

The Maurer-Cartan matrix becomes

$$
\begin{aligned}
d g \cdot g^{-1} & =\left(\begin{array}{ccc}
\overline{\mathrm{c}} d \mathrm{c}+\mathrm{c} d \overline{\mathrm{c}} & 0 & 0 \\
d \mathrm{~b} & d \mathrm{c} & 0 \\
d \mathrm{~d} & d \mathrm{e} & \frac{d \mathrm{c}}{\overline{\mathrm{c}}}-\frac{\mathrm{c} d \overline{\mathrm{c}}}{\overline{\mathrm{cc}}}
\end{array}\right)\left(\begin{array}{ccc}
\frac{1}{\mathrm{c}} & 0 & 0 \\
-\frac{\mathrm{b}}{\mathrm{cc} \bar{c}} & \frac{1}{\mathrm{c}} & 0 \\
\frac{\mathrm{be}-\mathrm{cd}}{\mathrm{ccc}} & -\frac{\overline{\mathrm{c}}}{\mathrm{cc}} & \frac{\overline{\mathrm{c}}}{\mathrm{c}}
\end{array}\right) \\
& =:\left(\begin{array}{ccc}
\alpha+\bar{\alpha} & 0 & 0 \\
\beta & \alpha & 0 \\
\gamma & \delta & \alpha-\bar{\alpha}
\end{array}\right),
\end{aligned}
$$

in terms of the group-invariant 1 -forms

$$
\begin{aligned}
\alpha & :=\frac{d \mathrm{c}}{\mathrm{c}}, \\
\beta & :=\frac{d \mathrm{~b}}{\mathrm{c} \overline{\mathrm{c}}}-\frac{\mathrm{b} d \mathrm{c}}{\mathrm{cc} \overline{\mathrm{c}}}, \\
\gamma & :=\frac{d \mathrm{~d}}{\mathrm{c} \overline{\mathrm{c}}}-\frac{\mathrm{b} d \mathrm{e}}{\mathrm{cc} \overline{\mathrm{c}}}+\frac{\mathrm{be}-\mathrm{cd}}{\mathrm{ccc} \overline{\mathrm{c}}} d \mathrm{c}-\frac{\mathrm{be}-\mathrm{cd}}{\mathrm{cc} \overline{c \mathrm{c}}} d \overline{\mathrm{c}}, \\
\delta & :=\frac{d \mathrm{e}}{\mathrm{c}}-\frac{\mathrm{e} d \mathrm{c}}{\mathrm{cc}}+\frac{\mathrm{e} d \overline{\mathrm{c}}}{\mathrm{c} \overline{\mathrm{c}}} .
\end{aligned}
$$

Now, let us exterior-differentiate the lifted coframe on the product manifold equipped with coordinates

$$
\left(z_{1}, z_{2}, \bar{z}_{1}, \bar{z}_{2}, v\right) \times(\mathrm{c}, \overline{\mathrm{c}}, \mathrm{b}, \overline{\mathrm{b}}, \mathrm{d}, \overline{\mathrm{d}}, \mathrm{e}, \overline{\mathrm{e}}) \in M^{5} \times G^{8}
$$


The computation starts as

$$
\begin{aligned}
& d \rho=(\overline{\mathrm{c}} d \mathrm{c}+\mathrm{c} d \overline{\mathrm{c}}) \wedge \rho_{0}+\mathrm{c} \overline{\mathrm{c}} d \rho_{0}, \\
& d \kappa=d \mathrm{~b} \wedge \rho_{0}+d \mathrm{c} \wedge \kappa_{0}+\mathrm{b} d \rho_{0}+\mathrm{c} d \kappa_{0}, \\
& d \zeta=d \mathrm{~d} \wedge \rho_{0}+d \mathrm{e} \wedge \kappa_{0}+\left(\frac{d \mathrm{c}}{\mathrm{c}}-\frac{\mathrm{c} d \overline{\mathrm{c}}}{\overline{\mathrm{cc}}}\right) \wedge \zeta_{0}^{\prime}+\mathrm{d} d \rho_{0}+\mathrm{e} d \kappa_{0}+\frac{\mathrm{c}}{\overline{\mathrm{c}}} d \zeta_{0}^{\prime} .
\end{aligned}
$$

As is known, one must replace in second lines $d \rho_{0}, d \kappa_{0}, d \zeta_{0}^{\prime}$ by the structure equations (5.5), and after, replace everywhere $\rho_{0}, \kappa_{0}, \zeta_{0}^{\prime}$, using the inversion formulas (6.1).

However, contrary to Pocchiola's systematic approach, we will not perform these calculations completely, but select only meaningful terms.

At least, at the level of Maurer-Cartan forms, after replacements of $\rho_{0}, \kappa_{0}, \zeta_{0}^{\prime}$ in the first lines of (6.2) above using (6.1), we have as usual

$$
\begin{aligned}
d \rho & =(\alpha+\bar{\alpha}) \wedge \rho+\text { Torsion, } \\
d \kappa & =\beta \wedge \rho+\alpha \wedge \kappa+\text { Torsion, } \\
d \zeta & =\gamma \wedge \rho+\delta \wedge \kappa+(\alpha-\bar{\alpha}) \wedge \zeta+\text { Torsion. }
\end{aligned}
$$

Question 6.3. Without computing everything, what are the shapes of the three Torsion remainders?

Consider for instance what happens of the last term $\frac{c}{\bar{c}} d \zeta_{0}^{\prime}$ in $d \zeta$, when performing the required replacements, and restrict attention even to the last term of $\frac{c}{\bar{c}} d \zeta_{0}^{\prime}$ in (5.5), which becomes

$$
\stackrel{\mathrm{c}}{\overline{\mathrm{c}}} \frac{\overline{\mathcal{L}}_{1}(\bar{k})}{\mathcal{L}_{1}(\bar{k})} \zeta_{0}^{\prime} \wedge \bar{\zeta}_{0}^{\prime}=\frac{\mathrm{c}}{\overline{\mathrm{c}}} \frac{\overline{\mathcal{L}}_{1}(\bar{k})}{\mathcal{L}_{1}(\bar{k})}\left(\frac{\mathrm{be}-\mathrm{cd}}{\mathrm{ccc}} \rho-\frac{\overline{\mathrm{ce}}}{\mathrm{cc}} \kappa+\frac{\overline{\mathrm{c}}}{\mathrm{c}} \zeta\right) \wedge\left(\frac{\overline{\mathrm{b}} \overline{\mathrm{e}}-\overline{\mathrm{cd}}}{\overline{\mathrm{cc}}} \rho-\frac{\mathrm{ce}}{\overline{\mathrm{cC}}} \bar{\kappa}+\frac{\mathrm{c}}{\overline{\mathrm{c}}} \bar{\zeta}\right) .
$$

After expansion, we see that are present the eight 2-forms
$(\bullet) \rho \wedge \kappa$,
$(\bullet) \rho \wedge \zeta$,
$(\bullet) \rho \wedge \bar{\kappa}$,
$(\bullet) \rho \wedge \bar{\zeta}$
$(\bullet) \kappa \wedge \bar{\kappa}$,
$(\bullet) \kappa \wedge \bar{\zeta}$
$(\cdot) \zeta \wedge \bar{\kappa}$
$(\bullet) \zeta \wedge \bar{\zeta}$

Doing the same for all torsion terms, we may realize - although it is not necessary to check this for what follows - with almost no computation that the nonexplicit shape of the structure equations of the lifted coframe is

$$
\begin{aligned}
d \rho= & (\alpha+\bar{\alpha}) \wedge \rho+R^{1} \rho \wedge \kappa+R^{2} \rho \wedge \zeta+\bar{R}^{1} \rho \wedge \bar{\kappa}+\bar{R}^{2} \rho \wedge \bar{\zeta}+i \kappa \wedge \bar{\kappa}, \\
d \kappa= & \beta \wedge \rho+\alpha \wedge \kappa+K^{1} \rho \wedge \kappa+K^{2} \rho \wedge \zeta+K^{3} \rho \wedge \bar{\kappa}+K^{4} \rho \wedge \bar{\zeta} \\
& +K^{5} \kappa \wedge \zeta+K^{6} \kappa \wedge \bar{\kappa}+\mathbf{1} \cdot \zeta \wedge \bar{\kappa}, \\
d \zeta= & \gamma \wedge \rho+\delta \wedge \kappa+(\alpha-\bar{\alpha}) \wedge \zeta \\
& +Z^{1} \rho \wedge \kappa+Z^{2} \rho \wedge \zeta+Z^{3} \rho \wedge \bar{\kappa}+Z^{4} \rho \wedge \bar{\zeta} \\
& +Z^{5} \kappa \wedge \zeta+Z^{6} \kappa \wedge \bar{\kappa}+Z^{7} \kappa \wedge \bar{\zeta}+Z^{8} \zeta \wedge \bar{\kappa}+Z^{9} \zeta \wedge \bar{\zeta} .
\end{aligned}
$$

Of course, the preceding normalization $\mathrm{f}:=\frac{\mathrm{c}}{\bar{c}} \overline{\mathcal{L}}_{1}(k)$ forces

$$
\mathbf{1}=[\zeta \wedge \bar{\kappa}]\{d \kappa\},
$$

a fact that can also be confirmed by a direct computation of this torsion coefficient (exercise).

So we do not compute all torsion coefficients like Pocchiola did, but we determine before some essential torsions, so that we may focus on just the useful torsion terms. In advance, we 
have boxed above the 3 useful ones, shown by Pocchiola. The subtle thing is that all three structure equations are needed.

Lemma 6.4. Here is an essential linear combination of torsion terms

$$
\bar{R}^{1}-2 K^{6}+Z^{8}
$$

Proof. In order to 'absorb' as many torsion coefficients as possible, let us substitute

$$
\begin{aligned}
& \alpha=: \alpha^{\prime}+a_{1} \rho+a_{2} \kappa+a_{3} \zeta+a_{4} \bar{\kappa}+a_{5} \bar{\zeta} \\
& \beta=: \beta^{\prime}+b_{1} \rho+b_{2} \kappa+b_{3} \zeta+b_{4} \bar{\kappa}+b_{5} \bar{\zeta} \\
& \gamma=: \gamma^{\prime}+c_{1} \rho+c_{2} \kappa+c_{3} \zeta+c_{4} \bar{\kappa}+c_{5} \bar{\zeta} \\
& \delta=: \delta^{\prime}+d_{1} \rho+d_{2} \kappa+d_{3} \zeta+d_{4} \bar{\kappa}+d_{5} \bar{\zeta}
\end{aligned}
$$

At first, we have to transform the structure equations after such a substitution, the task is easy, and we write out the details so that the reader needs no pen and no computer.

Substituting, the Maurer-Cartan part of $d \rho$ becomes

$$
\begin{aligned}
(\alpha+\bar{\alpha}) \wedge \rho= & \left(\alpha^{\prime}+\bar{\alpha}^{\prime}\right) \wedge \rho+0+a_{2} \kappa \wedge \rho+a_{3} \zeta \wedge \rho+a_{4} \bar{\kappa} \wedge \rho+a_{5} \bar{\zeta} \wedge \rho \\
& +0+\bar{a}_{2} \bar{\kappa} \wedge \rho+\bar{a}_{3} \bar{\zeta} \wedge \rho+\bar{a}_{4} \kappa \wedge \rho+\bar{a}_{5} \zeta \wedge \rho,
\end{aligned}
$$

hence adding and reorganizing visually, we get

$$
\begin{aligned}
d \rho= & \left(\alpha^{\prime}+\bar{\alpha}^{\prime}\right) \wedge \rho \\
& +\rho \wedge \kappa\left(R^{1}-a_{2}-\bar{a}_{4}\right)+\rho \wedge \zeta\left(R^{2}-a_{3}-\bar{a}_{5}\right)+\rho \wedge \bar{\kappa}\left(\bar{R}^{1}-a_{4}-\bar{a}_{2}\right) \\
& +\rho \wedge \bar{\zeta}\left(\bar{R}^{2}-a_{5}-\bar{a}_{3}\right)+i \kappa \wedge \bar{\kappa} .
\end{aligned}
$$

Next

$$
\begin{aligned}
\beta \wedge \rho+\alpha \wedge \kappa= & \beta^{\prime} \wedge \rho+0+b_{2} \kappa \wedge \rho+b_{3} \zeta \wedge \rho+b_{4} \bar{\kappa} \wedge \rho+b_{5} \bar{\zeta} \wedge \rho \\
& +\alpha^{\prime} \wedge \kappa+a_{1} \rho \wedge \kappa+0+a_{3} \zeta \wedge \kappa+a_{4} \bar{\kappa} \wedge \kappa+a_{5} \bar{\zeta} \wedge \kappa,
\end{aligned}
$$

hence

$$
\begin{aligned}
d \kappa= & \beta^{\prime} \wedge \rho+\alpha^{\prime} \wedge \kappa \\
& +\rho \wedge \kappa\left(K^{1}+a_{1}-b_{2}\right)+\rho \wedge \zeta\left(K^{2}-b_{3}\right)+\rho \wedge \bar{\kappa}\left(K^{3}-b_{4}\right)+\rho \wedge \bar{\zeta}\left(K^{4}-b_{5}\right) \\
& +\kappa \wedge \zeta\left(K^{5}-a_{3}\right)+\kappa \wedge \bar{\kappa}\left(K^{6}-a_{4}\right)+\kappa \wedge \bar{\zeta}\left(-a_{5}\right)+\zeta \wedge \bar{\kappa} .
\end{aligned}
$$

Lastly

$$
\begin{aligned}
\gamma \wedge \rho+\delta \wedge \kappa+(\alpha-\bar{\alpha}) \wedge \zeta= & \gamma^{\prime} \wedge \rho+0+c_{2} \kappa \wedge \rho+c_{3} \zeta \wedge \rho+c_{4} \bar{\kappa} \wedge \rho+c_{5} \bar{\zeta} \wedge \rho \\
& +\delta^{\prime} \wedge \kappa+d_{1} \rho \wedge \kappa+0+d_{3} \zeta \wedge \kappa+d_{4} \bar{\kappa} \wedge \kappa+d_{5} \bar{\zeta} \wedge \kappa \\
& +\alpha^{\prime} \wedge \zeta+a_{1} \rho \wedge \zeta+a_{2} \kappa \wedge \zeta+0+a_{4} \bar{\kappa} \wedge \zeta+a_{5} \bar{\zeta} \wedge \zeta \\
& -\bar{\alpha}^{\prime} \wedge \zeta-\bar{a}_{1} \rho \wedge \zeta-\bar{a}_{2} \bar{\kappa} \wedge \zeta-\bar{a}_{3} \bar{\zeta} \wedge \zeta-\bar{a}_{4} \kappa \wedge \zeta-0,
\end{aligned}
$$


hence

$$
\begin{aligned}
d \zeta= & \gamma^{\prime} \wedge \rho+\delta^{\prime} \wedge \kappa+\left(\alpha^{\prime}-\bar{\alpha}^{\prime}\right) \wedge \zeta \\
& +\rho \wedge \kappa\left(Z^{1}-c_{2}+d_{1}\right)+\rho \wedge \zeta\left(Z^{2}-c_{3}+a_{1}-\bar{a}_{1}\right)+\rho \wedge \bar{\kappa}\left(Z^{3}-c_{4}\right)+\rho \wedge \bar{\zeta}\left(Z^{4}-c_{5}\right) \\
& +\kappa \wedge \zeta\left(Z^{5}-d_{3}+a_{2}-\bar{a}_{4}\right)+\kappa \wedge \bar{\kappa}\left(Z^{6}-d_{4}\right)+\kappa \wedge \bar{\zeta}\left(Z^{7}-d_{5}\right) \\
& +\zeta \wedge \bar{\kappa}\left(Z^{8}-a_{4}+\bar{a}_{2}\right)+\zeta \wedge \bar{\zeta}\left(Z^{9}-a_{5}+\bar{a}_{3}\right) .
\end{aligned}
$$

Extracting the boxed three new torsion coefficients

$$
\begin{aligned}
\bar{R}^{1 \prime} & =\bar{R}^{1}-a_{4}-\bar{a}_{2}, \\
K^{6 \prime} & =K^{6}-a_{4}, \\
Z^{8 \prime} & =Z^{8}-a_{4}+\bar{a}_{2},
\end{aligned}
$$

we see well the announced essentiality/invariancy of this torsion combination

$$
\bar{R}^{1 \prime}-2 K^{6 \prime}+Z^{8 \prime}=\bar{R}^{1}-2 K^{6}+Z^{8}
$$

Consequently, we may restrict ourselves to computing only these three torsion coefficients.

Lemma 6.5. Their explicit expressions are

$$
\begin{aligned}
\bar{R}^{1} & =\frac{\bar{P}}{\overline{\mathrm{c}}}+\frac{\mathrm{c} \overline{\mathrm{e}}}{\overline{\mathrm{cc}}} \frac{\overline{\mathcal{L}}_{1}(\bar{k})}{\mathcal{L}_{1}(\bar{k})}-i \frac{\mathrm{b}}{\mathrm{c} \overline{\mathrm{c}}}, \\
K^{6} & =i \frac{\mathrm{b}}{\mathrm{c} \overline{\mathrm{c}}}-\frac{\mathrm{e}}{\mathrm{c}} \\
Z^{8} & =\frac{\mathrm{e}}{\mathrm{c}}-\frac{1}{\overline{\mathrm{c}}} \frac{\overline{\mathcal{L}}_{1}\left(\overline{\mathcal{L}}_{1}(k)\right)}{\overline{\mathcal{L}}_{1}(k)}-\frac{\mathrm{c} \overline{\mathrm{e}}}{\overline{\mathrm{cc}}} \frac{\overline{\mathcal{L}}_{1}(\bar{k})}{\mathcal{L}_{1}(\bar{k})} .
\end{aligned}
$$

Proof. We proceed by chasing coefficients. Let us treat $\bar{R}^{1}$. From (6.2), replacing in (5.5) by means of (6.1), we reach its expression

$$
\begin{aligned}
& \bar{R}^{1}=[\rho \wedge \bar{\kappa}]\left\{\mathrm{c} \overline{\mathrm{C}} d \rho_{0}\right\}=0+0+[\rho \wedge \bar{\kappa}]\left\{\mathrm{c} \overline{\mathrm{C}} \bar{P}\left(\frac{1}{\mathrm{c} \overline{\mathrm{C}}} \rho\right) \wedge\left(-\frac{\overline{\mathrm{b}}}{\overline{\mathrm{CC}}} \rho+\frac{1}{\overline{\mathrm{c}}} \bar{\kappa}\right)\right. \\
& -\mathrm{c} \overline{\mathrm{c}} \frac{\overline{\mathcal{L}}_{1}(\bar{k})}{\mathcal{L}_{1}(\bar{k})}\left(\frac{1}{\mathrm{c} \overline{\mathrm{c}}} \rho\right) \wedge\left(\frac{\overline{\mathrm{b}} \overline{\mathrm{e}}-\overline{\mathrm{c}} \overline{\mathrm{d}}}{\overline{\mathrm{ccc}}} \rho-\frac{\mathrm{c} \overline{\mathrm{e}}}{\overline{\mathrm{cc}}} \bar{\kappa}+\frac{\mathrm{c}}{\overline{\mathrm{c}}} \bar{\zeta}\right) \\
& \left.+\mathrm{c} \overline{\mathrm{c}} i\left(-\frac{\mathrm{b}}{\mathrm{cc} \overline{\mathrm{C}}} \rho+\frac{1}{\mathrm{c}} \kappa\right) \wedge\left(-\frac{\overline{\mathrm{b}}}{\mathrm{c} \overline{\mathrm{CC}}} \rho+\frac{1}{\overline{\mathrm{c}}} \bar{\kappa}\right)\right\} \\
& =\underline{\mathrm{c} \overline{\mathrm{C}}_{\circ}} \bar{P} \frac{1}{\underline{\mathrm{c} \overline{\mathrm{C}}_{\circ}}} \frac{1}{\overline{\mathrm{c}}}+\underline{\mathrm{c} \overline{\mathrm{C}}_{\circ}} \frac{\overline{\mathcal{L}}_{1}(\bar{k})}{\mathcal{L}_{1}(\bar{k})} \frac{1}{\underline{\mathrm{c} \overline{\mathrm{C}}_{\circ}}} \frac{\overline{\mathrm{ce}}}{\overline{\mathrm{cC}}}-i \underline{\mathrm{c} \overline{\mathrm{C}}_{\circ}} \frac{\mathrm{b}}{\mathrm{cc} \overline{\mathrm{C}}_{\circ}} \frac{1}{\overline{\mathrm{c}}} \text {. }
\end{aligned}
$$

Next, from (6.2), let us treat

$$
K^{6}=[\kappa \wedge \bar{\kappa}]\left\{\mathrm{b} d \rho_{0}+\mathrm{c} d \kappa_{0}\right\}
$$


In $\mathrm{b} d \rho_{0}$, the first four terms in (5.5) have zero contribution, since they are multiples of $\rho_{0}$, hence of $\rho$, whence

$$
\begin{aligned}
{[\kappa \wedge \bar{\kappa}]\left\{\mathrm{b} d \rho_{0}\right\} } & =0+0+0+0+[\kappa \wedge \bar{\kappa}]\left\{\mathrm{b} i \kappa_{0} \wedge \bar{\kappa}_{0}\right\} \\
& =[\kappa \wedge \bar{\kappa}]\left\{i \mathrm{~b}\left(-\frac{\mathrm{b}}{\mathrm{cc} \overline{\mathrm{C}}} \rho+\frac{1}{\mathrm{c}} \kappa\right) \wedge\left(-\frac{\overline{\mathrm{b}}}{\mathrm{c} \overline{\mathrm{CC}}} \rho+\frac{1}{\overline{\mathrm{c}}} \bar{\kappa}\right)\right\} \\
& =i \frac{\mathrm{b}}{\mathrm{c} \overline{\mathrm{C}}} .
\end{aligned}
$$

Also, in $\mathrm{c} d \kappa_{0}$, the first two terms contribute 0 , and it remains

$$
\begin{aligned}
{[\kappa \wedge \bar{\kappa}]\left\{\mathrm{c} d \kappa_{0}\right\} } & =0+0+[\kappa \wedge \bar{\kappa}]\left\{\mathrm{c} \zeta_{0}^{\prime} \wedge \bar{\kappa}_{0}\right\} \\
& =[\kappa \wedge \bar{\kappa}]\left\{\mathrm{c}\left(-\frac{\overline{\mathrm{ce}}}{\mathrm{cc}} \kappa\right) \wedge\left(\frac{1}{\overline{\mathrm{c}}} \bar{\kappa}\right)\right\} \\
& =-\frac{\mathrm{e}}{\mathrm{c}} .
\end{aligned}
$$

Lastly

$$
Z^{8}=[\zeta \wedge \bar{\kappa}]\left\{\mathrm{d} d \rho_{0}+\mathrm{e} d \kappa_{0}+\frac{\mathrm{c}}{\overline{\mathrm{c}}} d \zeta_{0}^{\prime}\right\}
$$

Here, $\mathrm{d} d \rho_{0}$ contributes 0 . Next, the first two terms in e $d \kappa_{0}$ contribute 0 , and it remains

$$
\begin{aligned}
{[\zeta \wedge \bar{\kappa}]\left\{\mathrm{e} d \kappa_{0}\right\} } & =[\zeta \wedge \bar{\kappa}]\left\{\mathrm{e} \zeta_{0}^{\prime} \wedge \kappa_{0}\right\} \\
& =[\zeta \wedge \bar{\kappa}]\left\{\mathrm{e}\left(\frac{\overline{\mathrm{c}}}{\mathrm{c}} \zeta\right) \wedge\left(\frac{1}{\overline{\mathrm{c}}} \bar{\kappa}\right)\right\} \\
& =\frac{\mathrm{e}}{\mathrm{c}} .
\end{aligned}
$$

Also, in $\frac{c}{\bar{c}} d \zeta_{0}^{\prime}$, the first two terms contribute 0 , and the last two terms are

$$
\begin{aligned}
{[\zeta \wedge \bar{\kappa}]\left\{\frac{\mathrm{c}}{\overline{\mathrm{c}}} d \zeta_{0}^{\prime}\right\}=} & -\frac{\mathrm{c}}{\overline{\mathrm{c}}} \frac{\overline{\mathcal{L}}_{1}\left(\overline{\mathcal{L}}_{1}(k)\right)}{\overline{\mathcal{L}}_{1}(k)}[\zeta \wedge \bar{\kappa}]\left\{\left(\frac{\overline{\mathrm{c}}}{\mathrm{c}} \zeta\right) \wedge\left(\frac{1}{\overline{\mathrm{c}}} \bar{\kappa}\right)\right\} \\
& +\frac{\mathrm{c}}{\overline{\mathrm{c}}} \frac{\overline{\mathcal{L}}_{1}(\bar{k})}{\mathcal{L}_{1}(\bar{k})}[\zeta \wedge \bar{\kappa}]\left\{\left(\frac{\overline{\mathrm{c}}}{\mathrm{c}} \zeta\right) \wedge\left(-\frac{\mathrm{c} \overline{\mathrm{e}}}{\overline{\mathrm{cc}}} \bar{\kappa}\right)\right\} \\
= & -\frac{1}{\overline{\mathrm{c}}} \frac{\overline{\mathcal{L}}_{1}\left(\overline{\mathcal{L}}_{1}(k)\right)}{\overline{\mathcal{L}}_{1}(k)}-\frac{\mathrm{c} \overline{\mathrm{e}}}{\overline{\overline{\mathrm{cc}}}} \frac{\overline{\mathcal{L}}_{1}(\bar{k})}{\mathcal{L}_{1}(\bar{k})}
\end{aligned}
$$

Adding, we get $Z^{8}$.

Observing that necessarily $-a_{5}=0$ from $[\kappa \wedge \bar{\zeta}]\{d \kappa\}$, we realize that some other invariant relations between torsion coefficients appear

$$
\begin{aligned}
& R^{2 \prime}-K^{5 \prime}=R^{2}-K^{5}, \\
& \bar{R}^{2 \prime}+Z^{9 \prime}=\bar{R}^{2}+Z^{9},
\end{aligned}
$$

that could potentially bring normalizations of some group parameters, but will not, as it will come out that they are identically satisfied. However, knowing them will be very useful later, hence we state a supplementary:

Assertion 6.6. Three other torsion coefficients have the common explicit expression

$$
R^{2}=K^{5}=-\bar{Z}^{9}=-\frac{\overline{\mathrm{c}}}{\mathrm{c}} \frac{\mathcal{L}_{1}(k)}{\overline{\mathcal{L}}_{1}(k)} .
$$


Proof. Our technique gives

$$
\begin{aligned}
R^{2} & =[\rho \wedge \zeta]\left\{c \bar{c} d \rho_{0}\right\} \\
& =0-c \bar{c} \frac{\mathcal{L}_{1}(k)}{\overline{\mathcal{L}}_{1}(k)} \frac{1}{c \bar{c}} \frac{\overline{\mathrm{c}}}{\mathrm{c}}+0+0+0 .
\end{aligned}
$$

Next

$$
\begin{aligned}
K^{5} & =[\zeta \wedge \kappa]\left\{\mathrm{b} d \rho_{0}+\mathrm{c} d \kappa_{0}\right\} \\
& =0+[\zeta \wedge \kappa]\left\{\mathrm{c} d \kappa_{0}\right\} \\
& =0-\frac{\mathcal{L}_{1}(k)}{\overline{\mathcal{L}_{1}(k)} \mathrm{c}} \mathrm{c} \frac{1 \overline{\mathrm{c}}}{\mathrm{c}} \mathrm{c}+0 .
\end{aligned}
$$

Lastly

$$
\begin{aligned}
& Z^{9}=[\bar{\kappa} \wedge \bar{\zeta}]\left\{\mathrm{d} d \rho_{0}+\mathrm{e} d \kappa_{0}+\frac{\mathrm{c}}{\overline{\mathrm{c}}} d \zeta_{0}^{\prime}\right\}
\end{aligned}
$$

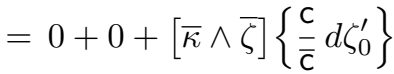

$$
\begin{aligned}
& =0+0+0+\frac{\mathrm{c}}{\overline{\mathrm{c}}} \frac{\overline{\mathcal{L}}_{1}(\bar{k})}{\mathcal{L}_{1}(\bar{k})} \frac{\bar{c}}{\mathrm{c}} \frac{\mathrm{c}}{\overline{\mathrm{c}}} .
\end{aligned}
$$

Coming back to Lemma 6.5, we can now compute in details, emphasizing one annihilation, the expression of the interesting invariant torsion combination

$$
\begin{aligned}
\bar{R}^{1}-2 K^{6}+Z^{8}= & \frac{\bar{P}}{\overline{\mathrm{c}}}+\frac{\overline{\mathrm{ce}} \frac{\overline{\mathcal{L}}_{1}(\bar{k})}{\overline{\mathrm{cc}}}}{\mathcal{L}_{1}(\bar{k})}-i \frac{\mathrm{b}}{\mathrm{c} \overline{\mathrm{c}}} \\
& -2 i \frac{\mathrm{b}}{\mathrm{c} \overline{\mathrm{c}}}+2 \frac{\mathrm{e}}{\mathrm{c}} \\
& +\frac{\mathrm{e}}{\mathrm{c}}-\frac{1}{\overline{\mathrm{c}}} \frac{\overline{\mathcal{L}}_{1}\left(\overline{\mathcal{L}}_{1}(k)\right)}{\overline{\mathcal{L}}_{1}(k)}-\frac{\mathrm{c} \overline{\overline{\mathrm{e}}} \overline{\mathcal{L}}_{1}(\bar{k})}{\overline{\mathrm{cc}} \frac{\overline{\mathcal{L}}_{1}(\bar{k})}{0}} \\
= & -3 i \frac{\mathrm{b}}{\mathrm{c} \overline{\mathrm{c}}}+3 \frac{\mathrm{e}}{\mathrm{c}}-\frac{1}{\overline{\mathrm{c}}}\left(\frac{\overline{\mathcal{L}}_{1}\left(\overline{\mathcal{L}}_{1}(k)\right)}{\overline{\mathcal{L}}_{1}(k)}-\bar{P}\right) .
\end{aligned}
$$

Since the group parameter $b \in \mathbb{C}$ is not on the diagonal, there is no restriction for it to be nonzero, hence we can normalize it by requiring that

$$
0=\bar{R}^{1}-2 K^{6}+Z^{8},
$$

and this produces the announced normalization

$$
\mathrm{b}:=-i \overline{\mathrm{ce}}+\frac{i}{3} \mathrm{c}\left(\frac{\overline{\mathcal{L}}_{1}\left(\overline{\mathcal{L}}_{1}(k)\right)}{\overline{\mathcal{L}}_{1}(k)}-\bar{P}\right) .
$$

For convenience, let us abbreviate

$$
B_{0}:=\frac{\overline{\mathcal{L}}_{1}\left(\overline{\mathcal{L}}_{1}(k)\right)}{\overline{\mathcal{L}}_{1}(k)}-\bar{P},
$$

which is function on $M$, as its lower index ${ }_{0}$ points out, so that

$$
\mathrm{b}:=-i \overline{\mathrm{c} e}+\frac{i}{3} \mathrm{c} B_{0} .
$$


After this normalization, the lifted coframe becomes

$$
\left(\begin{array}{c}
\rho \\
\kappa \\
\zeta
\end{array}\right):=\left(\begin{array}{ccc}
\mathrm{c} \overline{\mathrm{c}} & 0 & 0 \\
-i \overline{\mathrm{c} e}+\frac{i}{3} \mathrm{c} \boldsymbol{B}_{0} & \mathrm{c} & 0 \\
\mathrm{~d} & \mathrm{e} & \overline{\mathrm{c}}
\end{array}\right)\left(\begin{array}{c}
\rho_{0} \\
\kappa_{0} \\
\zeta_{0}^{\prime}
\end{array}\right) .
$$

Consequently, we can transform/rewrite in a natural way

$$
\begin{aligned}
\kappa & =\left(-i \overline{\mathrm{ce}}+\frac{i}{3} \mathrm{c} \boldsymbol{B}_{0}\right) \rho_{0}+\mathrm{c} \kappa_{0} \\
& =(-i \overline{\mathrm{c} e}) \rho_{0}+\mathrm{c}(\underbrace{\kappa_{0}+\frac{i}{3} B_{0} \rho_{0}}_{=: \kappa_{0}^{\prime}}),
\end{aligned}
$$

and this conducts us to change of initial coframe on $M$

$$
\left\{\rho_{0}, \kappa_{0}, \zeta_{0}^{\prime}, \bar{\kappa}_{0}, \bar{\zeta}_{0}^{\prime}\right\} \rightsquigarrow\left\{\rho_{0}, \kappa_{0}^{\prime}, \zeta_{0}^{\prime}, \bar{\kappa}_{0}^{\prime}, \bar{\zeta}_{0}^{\prime}\right\},
$$

by introducing

$$
\kappa_{0}^{\prime}:=\kappa_{0}+\frac{i}{3} \boldsymbol{B}_{0} \rho_{0}
$$

It follows that

$$
\begin{aligned}
\zeta=\mathrm{d} \rho_{0}+\mathrm{e} \kappa_{0}+\frac{\mathrm{c}}{\overline{\mathrm{c}}} \zeta_{0}^{\prime} & =\mathrm{d} \rho_{0}+\mathrm{e}\left(\kappa_{0}^{\prime}-\frac{i}{3} B_{0} \rho_{0}\right)+\frac{\mathrm{c}}{\overline{\mathrm{c}}} \zeta_{0}^{\prime} \\
& =(\underbrace{\mathrm{d}-\frac{i}{3} \mathrm{e} B_{0}}_{=: \mathrm{d}^{\prime}}) \rho_{0}+\mathrm{e} \kappa_{0}^{\prime}+\frac{\mathrm{c}}{\overline{\mathrm{c}}} \zeta_{0}^{\prime} .
\end{aligned}
$$

Before, $d \in \mathbb{C}$ was a parameter representing some unknown function. Introducing the new unknown/parameter

$$
\mathrm{d}^{\prime}:=\mathrm{d}-\frac{i}{3} \mathrm{e},
$$

we come to a new $G$-structure of real dimension 6 parametrized by c, e $\in \mathbb{C}^{*}$ and $\mathrm{d}^{\prime} \in \mathbb{C}$ whose lifted coframe writes

$$
\left(\begin{array}{c}
\rho \\
\kappa \\
\zeta
\end{array}\right):=\left(\begin{array}{ccc}
\mathrm{c} \overline{\mathrm{c}} & 0 & 0 \\
-i \overline{\mathrm{ce}} & \mathrm{c} & 0 \\
\mathrm{~d}^{\prime} & \mathrm{e} & \overline{\mathrm{c}}
\end{array}\right)\left(\begin{array}{c}
\rho_{0} \\
\kappa_{0}^{\prime} \\
\zeta_{0}^{\prime}
\end{array}\right) .
$$

We will write again $d$ instead of $d^{\prime}$.

\section{Darboux-Cartan Structure of The Coframe $\left\{\rho_{0}, \kappa_{0}^{\prime}, \zeta_{0}^{\prime}, \bar{\kappa}_{0}^{\prime}, \bar{\zeta}_{0}^{\prime}\right\}$}

Before continuing, we must compute the Darboux-Cartan structure of this new initial coframe $\left\{\rho_{0}, \kappa_{0}^{\prime}, \zeta_{0}^{\prime}, \bar{\kappa}_{0}^{\prime}, \bar{\zeta}_{0}^{\prime}\right\}$, for which absolutely no details were provided in $[25,18]$. Here, we offer complete explanations.

Abstractly, the structure in question will have the shape

$$
\begin{aligned}
d \rho_{0}= & R_{0}^{1 \prime} \rho_{0} \wedge \kappa_{0}^{\prime}+R_{0}^{2 \prime} \rho_{0} \wedge \zeta_{0}^{\prime}+\bar{R}_{0}^{1 \prime} \rho_{0} \wedge \bar{\kappa}_{0}^{\prime}+\bar{R}_{0}^{2 \prime} \rho_{0} \wedge \bar{\zeta}_{0}^{\prime}+i \kappa_{0}^{\prime} \wedge \bar{\kappa}_{0}^{\prime}, \\
d \kappa_{0}^{\prime}= & K_{0}^{1 \prime} \rho_{0} \wedge \kappa_{0}^{\prime}+K_{0}^{2 \prime} \rho_{0} \wedge \zeta_{0}^{\prime}+K_{0}^{3 \prime} \rho_{0} \wedge \bar{\kappa}_{0}^{\prime} \\
& +K_{0}^{5 \prime} \kappa_{0}^{\prime} \wedge \zeta_{0}^{\prime}+K_{0}^{6 \prime} \kappa_{0}^{\prime} \wedge \bar{\kappa}_{0}^{\prime}+\zeta_{0}^{\prime} \wedge \bar{\kappa}_{0}^{\prime}, \\
d \zeta_{0}^{\prime}= & Z_{0}^{2 \prime} \rho_{0} \wedge \zeta_{0}^{\prime}+Z_{0}^{5 \prime} \kappa_{0}^{\prime} \wedge \zeta_{0}^{\prime}+Z_{0}^{8 \prime} \zeta_{0}^{\prime} \wedge \bar{\kappa}_{0}^{\prime}+Z_{0}^{9 \prime} \zeta_{0}^{\prime} \wedge \bar{\zeta}_{0}^{\prime} .
\end{aligned}
$$

Our goal is to compute explicitly all these coefficients, and the answer is stated as follows: 
Proposition 7.2. The Darboux-Cartan structure for the initial coframe $\left\{\rho_{0}, \kappa_{0}^{\prime}, \zeta_{0}^{\prime}, \bar{\kappa}_{0}^{\prime}, \bar{\zeta}_{0}^{\prime}\right\}$ expands as

$$
\begin{aligned}
& d \rho_{0}=\left(\frac{1}{3} \frac{\mathcal{L}_{1}\left(\mathcal{L}_{1}(\bar{k})\right)}{\mathcal{L}_{1}(\bar{k})}+\frac{2}{3} P\right) \rho_{0} \wedge \kappa_{0}^{\prime}-\frac{\mathcal{L}_{1}(k)}{\overline{\mathcal{L}}_{1}(k)} \rho_{0} \wedge \zeta_{0}^{\prime} \\
& +\left(\frac{1}{3} \frac{\overline{\mathcal{L}}_{1}\left(\overline{\mathcal{L}}_{1}(k)\right)}{\overline{\mathcal{L}}_{1}(k)}+\frac{2}{3} \bar{P}\right) \rho_{0} \wedge \bar{\kappa}_{0}^{\prime}-\frac{\overline{\mathcal{L}}_{1}(\bar{k})}{\mathcal{L}_{1}(\bar{k})} \rho_{0} \wedge \bar{\zeta}_{0}^{\prime}+i \kappa_{0}^{\prime} \wedge \bar{\kappa}_{0}^{\prime}, \\
& d \kappa_{0}^{\prime}=\left(-\frac{i}{3} \frac{\mathcal{L}_{1}\left(\overline{\mathcal{L}}_{1}\left(\overline{\mathcal{L}}_{1}(k)\right)\right)}{\overline{\mathcal{L}}_{1}(k)}+\frac{i}{9} \frac{\mathcal{L}_{1}\left(\mathcal{L}_{1}(\bar{k})\right) \overline{\mathcal{L}}_{1}\left(\overline{\mathcal{L}}_{1}(k)\right)}{\mathcal{L}_{1}(\bar{k}) \overline{\mathcal{L}}_{1}(k)}\right. \\
& +\frac{i}{3} \frac{\mathcal{L}_{1}\left(\overline{\mathcal{L}}_{1}(k)\right) \overline{\mathcal{L}}_{1}\left(\overline{\mathcal{L}}_{1}(k)\right)}{\overline{\mathcal{L}}_{1}(k)^{2}}-\frac{i}{9} \frac{\mathcal{L}_{1}\left(\mathcal{L}_{1}(\bar{k})\right)}{\mathcal{L}_{1}(\bar{k})} \bar{P} \\
& \left.+\frac{2 i}{9} \frac{\overline{\mathcal{L}}_{1}\left(\overline{\mathcal{L}}_{1}(k)\right)}{\overline{\mathcal{L}}_{1}(k)} P+\frac{i}{3} \mathcal{L}_{1}(\bar{P})-\frac{2 i}{9} P \bar{P}\right) \rho_{0} \wedge \kappa_{0}^{\prime} \\
& +\left(-\frac{i}{3} \frac{\mathcal{K}\left(\overline{\mathcal{L}}_{1}\left(\overline{\mathcal{L}}_{1}(k)\right)\right)}{\overline{\mathcal{L}}_{1}(k)^{2}}+\frac{i}{3} \frac{\mathcal{K}\left(\overline{\mathcal{L}}_{1}(k)\right) \overline{\mathcal{L}}_{1}\left(\overline{\mathcal{L}}_{1}(k)\right)}{\overline{\mathcal{L}}_{1}(k)^{3}}\right. \\
& \left.-\frac{i}{3} \frac{\mathcal{L}_{1}\left(\mathcal{L}_{1}(\bar{k})\right)}{\mathcal{L}_{1}(\bar{k})}-\frac{i}{3} \frac{\overline{\mathcal{L}}_{1}\left(\mathcal{L}_{1}(k)\right)}{\overline{\mathcal{L}}_{1}(k)}-\frac{2}{3} \frac{\mathcal{T}(k)}{\overline{\mathcal{L}}_{1}(k)}\right) \rho_{0} \wedge \zeta_{0}^{\prime} \\
& +\left(-\frac{i}{3} \frac{\overline{\mathcal{L}}_{1}\left(\overline{\mathcal{L}}_{1}\left(\overline{\mathcal{L}}_{1}(k)\right)\right)}{\overline{\mathcal{L}}_{1}(k)}+\frac{4 i}{9} \frac{\overline{\mathcal{L}}_{1}\left(\overline{\mathcal{L}}_{1}(k)\right)^{2}}{\overline{\mathcal{L}}_{1}(k)^{2}}\right. \\
& \left.+\frac{i}{9} \frac{\overline{\mathcal{L}}_{1}\left(\overline{\mathcal{L}}_{1}(k)\right)}{\overline{\mathcal{L}}_{1}(k)} \overline{\boldsymbol{P}}+\frac{i}{3} \overline{\mathcal{L}}_{1}(\bar{P})-\frac{2 i}{9} \overline{\boldsymbol{P}} \overline{\boldsymbol{P}}\right) \rho_{0} \wedge \bar{\kappa}_{0}^{\prime}+0 \rho_{0} \wedge \bar{\zeta}_{0}^{\prime} \\
& -\frac{\mathcal{L}_{1}(k)}{\overline{\mathcal{L}}_{1}(k)} \kappa_{0}^{\prime} \wedge \zeta_{0}^{\prime}+\left(-\frac{1}{3} \frac{\overline{\mathcal{L}}_{1}\left(\overline{\mathcal{L}}_{1}(k)\right)}{\overline{\mathcal{L}}_{1}(k)}+\frac{1}{3} \bar{P}\right) \kappa_{0}^{\prime} \wedge \bar{\kappa}_{0}^{\prime}+\zeta_{0}^{\prime} \wedge \bar{\kappa}_{0}^{\prime}, \\
& d \zeta_{0}^{\prime}=\left(\frac{i}{3} \frac{\mathcal{L}_{1}\left(\mathcal{L}_{1}(\bar{k})\right) \overline{\mathcal{L}}_{1}\left(\overline{\mathcal{L}}_{1}(k)\right)}{\mathcal{L}_{1}(\bar{k}) \overline{\mathcal{L}}_{1}(k)}-\frac{i}{3} \frac{\mathcal{L}_{1}\left(\overline{\mathcal{L}}_{1}(k)\right) \overline{\mathcal{L}}_{1}\left(\overline{\mathcal{L}}_{1}(k)\right)}{\overline{\mathcal{L}}_{1}(k)^{2}}\right. \\
& \left.-\frac{i}{3} \frac{\overline{\mathcal{L}}_{1}\left(\overline{\mathcal{L}}_{1}(k)\right)}{\overline{\mathcal{L}}_{1}(k)} P+\frac{i}{3} \frac{\mathcal{L}_{1}\left(\overline{\mathcal{L}}_{1}(k)\right)}{\overline{\mathcal{L}}_{1}(k)} \bar{P}+\frac{\mathcal{T}\left(\overline{\mathcal{L}}_{1}(k)\right)}{\overline{\mathcal{L}}_{1}(k)}\right) \rho_{0} \wedge \zeta_{0}^{\prime} \\
& +\frac{\mathcal{L}_{1}\left(\overline{\mathcal{L}}_{1}(k)\right)}{\overline{\mathcal{L}}_{1}(k)} \kappa_{0}^{\prime} \wedge \zeta_{0}^{\prime}-\frac{\overline{\mathcal{L}}_{1}\left(\overline{\mathcal{L}}_{1}(k)\right)}{\overline{\mathcal{L}}_{1}(k)} \zeta_{0}^{\prime} \wedge \bar{\kappa}_{0}^{\prime}+\frac{\overline{\mathcal{L}}_{1}(\bar{k})}{\mathcal{L}_{1}(\bar{k})} \zeta_{0}^{\prime} \wedge \bar{\zeta}_{0}^{\prime} .
\end{aligned}
$$

Observe from these explicit expressions that

$$
2 K_{0}^{6 \prime}=\bar{R}_{0}^{1 \prime}+Z_{0}^{8 \prime} \quad \text { and } \quad R_{0}^{2 \prime}=K_{0}^{5 \prime} .
$$

Proof. We treat first $d \rho_{0}$ and $d \zeta_{0}^{\prime}$, which are easier than $d \kappa_{0}^{\prime}$. Observing from (6.8), that

$$
\rho_{0} \wedge \kappa_{0}=\rho_{0} \wedge \kappa_{0}^{\prime} \quad \text { and } \quad \rho_{0} \wedge \bar{\kappa}_{0}=\rho_{0} \wedge \bar{\kappa}_{0}^{\prime},
$$


it comes by replacement in (5.5)

$$
\begin{aligned}
d \rho_{0}= & P \rho_{0} \wedge \kappa_{0}^{\prime}-\frac{\mathcal{L}_{1}(k)}{\overline{\mathcal{L}}_{1}(k)} \rho_{0} \wedge \zeta_{0}^{\prime}+\bar{P} \rho_{0} \wedge \bar{\kappa}_{0}-\frac{\overline{\mathcal{L}}_{1}(\bar{k})}{\mathcal{L}_{1}(\bar{k})} \rho_{0} \wedge \bar{\zeta}_{0}^{\prime} \\
& +i\left(\kappa_{0}^{\prime}-\frac{i}{3}\left(\frac{\overline{\mathcal{L}}_{1}\left(\overline{\mathcal{L}}_{1}(k)\right)}{\overline{\mathcal{L}}_{1}(k)}-\bar{P}\right) \rho_{0}\right) \wedge\left(\bar{\kappa}_{0}^{\prime}+\frac{i}{3}\left(\frac{\mathcal{L}_{1}\left(\mathcal{L}_{1}(\bar{k})\right)}{\mathcal{L}_{1}(\bar{k})}\right) \rho_{0}\right)
\end{aligned}
$$

and a plain expansion yields the stated expression of $d \rho_{0}$. Next, again from (6.8), it comes by replacement in (5.5)

$$
\begin{aligned}
d \zeta_{0}^{\prime}= & \frac{\mathcal{T}\left(\overline{\mathcal{L}}_{1}(k)\right)}{\overline{\mathcal{L}}_{1}(k)} \rho_{0} \wedge \zeta_{0}^{\prime}+\frac{\mathcal{L}_{1}\left(\overline{\mathcal{L}}_{1}(k)\right)}{\overline{\mathcal{L}}_{1}(k)}\left(\kappa_{0}^{\prime}-\frac{i}{3}\left(\frac{\overline{\mathcal{L}}_{1}\left(\overline{\mathcal{L}}_{1}(k)\right)}{\overline{\mathcal{L}}_{1}(k)}-\bar{P}\right) \rho_{0}\right) \wedge \zeta_{0}^{\prime} \\
& -\frac{\overline{\mathcal{L}}_{1}\left(\overline{\mathcal{L}}_{1}(k)\right)}{\overline{\mathcal{L}}_{1}(k)} \zeta_{0}^{\prime} \wedge\left(\bar{\kappa}_{0}^{\prime}+\frac{i}{3}\left(\frac{\mathcal{L}_{1}\left(\mathcal{L}_{1}(\bar{k})\right)}{\mathcal{L}_{1}(\bar{k})}-P\right) \rho_{0}\right)+\frac{\overline{\mathcal{L}}_{1}(\bar{k})}{\mathcal{L}_{1}(\bar{k})} \zeta_{0}^{\prime} \wedge \bar{\zeta}_{0}^{\prime}
\end{aligned}
$$

and visually - no pen needed - , we obtain the stated result. To treat $d \kappa_{0}^{\prime}$, we start from

$$
\kappa_{0}^{\prime}=\kappa_{0}+\frac{i}{3} \boldsymbol{B}_{0} \rho_{0}
$$

and we exterior differentiate

$$
d \kappa_{0}^{\prime}=d \kappa_{0}+\frac{i}{3} d \boldsymbol{B}_{0} \wedge \rho_{0}+\frac{i}{3} \boldsymbol{B}_{0} d \rho_{0} .
$$

As a preliminary, we need to know $d \boldsymbol{B}_{0}$. Let us recall that

$$
B_{0}=\frac{\overline{\mathcal{L}}_{1}\left(\overline{\mathcal{L}}_{1}(k)\right)}{\overline{\mathcal{L}}_{1}(k)}-\bar{P} \quad \text { whence } \quad \bar{B}_{0}=\frac{\mathcal{L}_{1}\left(\mathcal{L}_{1}(\bar{k})\right)}{\mathcal{L}_{1}(\bar{k})}-P .
$$

A plain application of Lemma 2.6 provides this exterior differential

$$
\begin{aligned}
& d\left(\frac{\overline{\mathcal{L}}_{1}\left(\overline{\mathcal{L}}_{1}(k)\right)}{\overline{\mathcal{L}}_{1}(k)}-\bar{P}\right)=\left(\frac{\mathcal{T}\left(\overline{\mathcal{L}}_{1}\left(\overline{\mathcal{L}}_{1}(k)\right)\right)}{\overline{\mathcal{L}}_{1}(k)}-\frac{\mathcal{T}\left(\overline{\mathcal{L}}_{1}(k)\right) \overline{\mathcal{L}}_{1}\left(\overline{\mathcal{L}}_{1}(k)\right)}{\overline{\mathcal{L}}_{1}(k)^{2}}-\mathcal{T}(\bar{P})\right) \rho_{0} \\
& +\left(\frac{\mathcal{L}_{1}\left(\overline{\mathcal{L}}_{1}\left(\overline{\mathcal{L}}_{1}(k)\right)\right)}{\overline{\mathcal{L}}_{1}(k)}-\frac{\mathcal{L}_{1}\left(\overline{\mathcal{L}}_{1}(k)\right) \overline{\mathcal{L}}_{1}\left(\overline{\mathcal{L}}_{1}(k)\right)}{\overline{\mathcal{L}}_{1}(k)^{2}}-\mathcal{L}_{1}(\bar{P})\right) \kappa_{0} \\
& +\left(\frac{\mathcal{K}\left(\overline{\mathcal{L}}_{1}\left(\overline{\mathcal{L}}_{1}(k)\right)\right)}{\overline{\mathcal{L}}_{1}(k)}-\frac{\mathcal{K}\left(\overline{\mathcal{L}}_{1}(k)\right) \overline{\mathcal{L}}_{1}\left(\overline{\mathcal{L}}_{1}(k)\right)}{\overline{\mathcal{L}}_{1}(k)^{2}}-\mathcal{K}(\bar{P})\right) \zeta_{0} \\
& +\left(\frac{\overline{\mathcal{L}}_{1}\left(\overline{\mathcal{L}}_{1}\left(\overline{\mathcal{L}}_{1}(k)\right)\right)}{\overline{\mathcal{L}}_{1}(k)}-\frac{\overline{\mathcal{L}}_{1}\left(\overline{\mathcal{L}}_{1}(k)\right)^{2}}{\overline{\mathcal{L}}_{1}(k)^{2}}-\overline{\mathcal{L}}_{1}(\bar{P})\right) \bar{\kappa}_{0} \\
& +\left(\frac{\overline{\mathcal{K}}\left(\overline{\mathcal{L}}_{1}\left(\overline{\mathcal{L}}_{1}(k)\right)\right)}{\overline{\mathcal{L}}_{1}(k)}-\frac{\overline{\mathcal{K}}\left(\overline{\mathcal{L}}_{1}(k)\right) \overline{\mathcal{L}}_{1}\left(\overline{\mathcal{L}}_{1}(k)\right)}{\overline{\mathcal{L}}_{1}(k)^{2}}-\overline{\mathcal{K}}(\bar{P})\right) \bar{\zeta}_{0},
\end{aligned}
$$

an expression that we will abbreviate as

$$
d \boldsymbol{B}_{0}=U_{0} \rho_{0}+V_{0} \kappa_{0}+W_{0} \zeta_{0}+X_{0} \bar{\kappa}_{0}+Y_{0} \bar{\zeta}_{0} .
$$

Assertion 7.4. After simplifications

$$
Y_{0}=-\frac{\overline{\mathcal{L}}_{1}(\bar{k}) \overline{\mathcal{L}}_{1}\left(\overline{\mathcal{L}}_{1}(k)\right)}{\overline{\mathcal{L}}_{1}(k)}+\overline{\mathcal{L}}_{1}(\bar{k}) \bar{P}
$$


Proof. In the first two terms of $Y_{0}$, we replace from Lemma 2.9

$$
\begin{aligned}
\overline{\mathcal{K}}\left(\overline{\mathcal{L}}_{1}\left(\overline{\mathcal{L}}_{1}(k)\right)\right) & =-2 \overline{\mathcal{L}}_{1}(\bar{k}) \overline{\mathcal{L}}_{1}\left(\overline{\mathcal{L}}_{1}(k)\right)-\overline{\mathcal{L}}_{1}\left(\overline{\mathcal{L}}_{1}(\bar{k})\right) \overline{\mathcal{L}}_{1}(k), \\
\overline{\mathcal{K}}\left(\overline{\mathcal{L}}_{1}(k)\right) & =-\overline{\mathcal{L}}_{1}(\bar{k}) \overline{\mathcal{L}}_{1}(k)
\end{aligned}
$$

and in the third term of $Y_{0}$, we replace from Lemma 2.5

$$
\overline{\mathcal{K}}(\bar{P})=-\bar{P} \overline{\mathcal{L}}_{1}(\bar{k})-\overline{\mathcal{L}}_{1}\left(\overline{\mathcal{L}}_{1}(\bar{k})\right),
$$

which yields the result after one (underlined) pair cancellation

$$
\begin{aligned}
& Y_{0}=-\frac{2 \overline{\mathcal{L}}_{1}(\bar{k}) \overline{\mathcal{L}}_{1}\left(\overline{\mathcal{L}}_{1}(k)\right)}{\overline{\mathcal{L}}_{1}(k)}-{\underline{\mathcal{L}_{1}\left(\overline{\mathcal{L}}_{1}(\bar{k})\right)}}_{\circ}+\frac{\overline{\mathcal{L}}_{1}(\bar{k}) \overline{\mathcal{L}}_{1}\left(\overline{\mathcal{L}}_{1}(k)\right)}{\overline{\mathcal{L}}_{1}(k)} \\
& +\bar{P} \overline{\mathcal{L}}_{1}(\bar{k})+\underline{\overline{\mathcal{L}}_{1}\left(\overline{\mathcal{L}}_{1}(\bar{k})\right)} .
\end{aligned}
$$

Temporarily, let us work with the abbreviations $U_{0}, V_{0}, W_{0}, X_{0}, Y_{0}$. So, using the previous structure formulas (5.5) in which, directly we replace

$$
\zeta_{0}=\frac{\zeta_{0}^{\prime}}{\overline{\mathcal{L}}_{1}(k)},
$$

let us add line-by-line all three terms of (7.3)

$$
\begin{aligned}
d \kappa_{0}^{\prime}= & -\frac{\mathcal{T}(k)}{\overline{\mathcal{L}}_{1}(k)} \rho_{0} \wedge \zeta_{0}^{\prime}-\frac{\mathcal{L}_{1}(k)}{\overline{\mathcal{L}}_{1}(k)} \kappa_{0} \wedge \zeta_{0}^{\prime}+\zeta_{0}^{\prime} \wedge \bar{\kappa}_{0}^{\prime} \\
& +\frac{i}{3} \frac{U_{0} \rho_{0} \wedge \rho_{0}}{\circ}+\frac{i}{3} V_{0} \kappa_{0} \wedge \rho_{0}+\frac{i}{3} W_{0} \frac{\zeta_{0}^{\prime}}{\overline{\mathcal{L}}_{1}(k)} \wedge \rho_{0}+\frac{i}{3} X_{0} \bar{\kappa}_{0} \wedge \rho_{0}+\frac{i}{3} Y_{0} \frac{\bar{\zeta}_{0}^{\prime}}{\mathcal{L}_{1}(\bar{k})} \wedge \rho_{0} \\
& +\frac{i}{3} B_{0} P \rho_{0} \wedge \kappa_{0}-\frac{i}{3} B_{0} \frac{\mathcal{L}_{1}(k)}{\overline{\mathcal{L}}_{1}(k)} \rho_{0} \wedge \zeta_{0}^{\prime}+\frac{i}{3} B_{0} \bar{P} \rho_{0} \wedge \bar{\kappa}_{0}-\frac{i}{3} B_{0} \frac{\overline{\mathcal{L}}_{1}(\bar{k})}{\mathcal{L}_{1}(\bar{k})} \rho_{0} \wedge \bar{\zeta}_{0}^{\prime}-\frac{1}{3} B_{0} \kappa_{0} \wedge \bar{\kappa}_{0},
\end{aligned}
$$

hence after collecting coefficients of basic 2-forms, we get

$$
\begin{aligned}
d \kappa_{0}^{\prime}= & \rho_{0} \wedge \zeta_{0}^{\prime}\left[-\frac{\mathcal{T}(k)}{\overline{\mathcal{L}}_{1}(k)}-\frac{i}{3} \frac{W_{0}}{\overline{\mathcal{L}}_{1}(k)}-\frac{i}{3} B_{0} \frac{\mathcal{L}_{1}(k)}{\overline{\mathcal{L}}_{1}(k)}\right]+\rho_{0} \wedge \kappa_{0}\left[-\frac{i}{3} V_{0}+\frac{i}{3} B_{0} P\right] \\
& +\rho_{0} \wedge \bar{\kappa}_{0}\left[-\frac{i}{3} X_{0}+\frac{i}{3} B_{0} \bar{P}\right]+\rho_{0} \wedge \bar{\zeta}_{0}^{\prime}\left[-\frac{i}{3} \frac{Y_{0}}{\mathcal{L}_{1}(\bar{k})}-\frac{i}{3} B_{0} \frac{\overline{\mathcal{L}}_{1}(\bar{k})}{\mathcal{L}_{1}(\bar{k})}\right] \\
& +\kappa_{0} \wedge \zeta_{0}^{\prime}\left[-\frac{\mathcal{L}_{1}(k)}{\overline{\mathcal{L}}_{1}(k)}\right]+\kappa_{0} \wedge \bar{\kappa}_{0}\left[-\frac{1}{3} B_{0}\right]+\zeta_{0}^{\prime} \wedge \bar{\kappa}_{0} .
\end{aligned}
$$

Next, replace everywhere

$$
\kappa_{0}=\kappa_{0}^{\prime}-\frac{i}{3} \boldsymbol{B}_{0} \rho_{0} .
$$

Then using again $\kappa_{0} \wedge \rho_{0}=\kappa_{0}^{\prime} \wedge \rho_{0}$, only the last line changes, as it becomes

$$
\left(\kappa_{0}^{\prime}-\frac{i}{3} B_{0} \rho_{0}\right) \wedge \zeta_{0}^{\prime}\left[-\frac{\mathcal{L}_{1}(k)}{\overline{\mathcal{L}}_{1}(k)}\right]+\left(\kappa_{0}^{\prime}-\frac{i}{3} \boldsymbol{B}_{0} \rho_{0}\right) \wedge\left(\bar{\kappa}_{0}^{\prime}+\frac{i}{3} \overline{\boldsymbol{B}}_{0} \rho_{0}\right)\left[-\frac{1}{3} \boldsymbol{B}_{0}\right]+\zeta_{0}^{\prime} \wedge\left(\bar{\kappa}_{0}^{\prime}+\frac{i}{3} \overline{\boldsymbol{B}}_{0} \rho_{0}\right) .
$$


Expanding and collecting visually — no pen needed —, we get

$$
\begin{aligned}
d \kappa_{0}^{\prime}= & \rho_{0} \wedge \zeta_{0}^{\prime}\left[-\frac{\mathcal{T}(k)}{\overline{\mathcal{L}}_{1}(k)}-\frac{i}{3} \frac{W_{0}}{\overline{\mathcal{L}}_{1}(k)}-\frac{i}{3} B_{0} \frac{\mathcal{L}_{1}(k)}{\overline{\mathcal{L}}_{1}(k)} \circ+\frac{i}{3} B_{0} \frac{\mathcal{L}_{1}(k)}{\overline{\mathcal{L}}_{1}(k)} \circ-\frac{i}{3} \bar{B}_{0}\right] \\
& +\rho_{0} \wedge \kappa_{0}^{\prime}\left[-\frac{i}{3} V_{0}+\frac{i}{3} B_{0} P+\frac{i}{9} B_{0} \bar{B}_{0}\right] \\
& +\rho_{0} \wedge \bar{\kappa}_{0}^{\prime}\left[-\frac{i}{3} X_{0}+\frac{i}{3} B_{0} \bar{P}+\frac{i}{9} B_{0} B_{0}\right] \\
& +\rho_{0} \wedge \bar{\zeta}_{0}^{\prime}\left[-\frac{i}{3} \frac{Y_{0}}{\mathcal{L}_{1}(\bar{k})}-\frac{i}{3} B_{0} \frac{\overline{\mathcal{L}}_{1}(\bar{k})}{\mathcal{L}_{1}(\bar{k})} 。\right] \\
& +\kappa_{0}^{\prime} \wedge \zeta_{0}^{\prime}\left[-\frac{\mathcal{L}_{1}(k)}{\overline{\mathcal{L}}_{1}(k)}\right]+\kappa_{0}^{\prime} \wedge \bar{\kappa}_{0}^{\prime}\left[-\frac{1}{3} B_{0}\right]+\zeta_{0}^{\prime} \wedge \bar{\kappa}_{0}^{\prime} .
\end{aligned}
$$

To finish, we must yet replace $V_{0}, W_{0}, X_{0}, Y_{0}$ by their complete values, and we will realize, as indicated by anticipation above, that the coefficient of $\rho_{0} \wedge \bar{\zeta}_{0}^{\prime}$ vanishes identically.

Firstly, a replacement followed by a visual expansion finalizes

$$
\begin{aligned}
{\left[\rho_{0} \wedge \kappa_{0}^{\prime}\right]\left\{d \kappa_{0}^{\prime}\right\}=} & -\frac{i}{3} \frac{\mathcal{L}_{1}\left(\overline{\mathcal{L}}_{1}\left(\overline{\mathcal{L}}_{1}(k)\right)\right)}{\overline{\mathcal{L}}_{1}(k)}+\frac{i}{3} \frac{\mathcal{L}_{1}\left(\overline{\mathcal{L}}_{1}(k)\right) \overline{\mathcal{L}}_{1}\left(\overline{\mathcal{L}}_{1}(k)\right)}{\overline{\mathcal{L}}_{1}(k)^{2}}+\frac{i}{3} \mathcal{L}_{1}(\bar{P}) \\
& +\frac{i}{3} \frac{\overline{\mathcal{L}}_{1}\left(\overline{\mathcal{L}}_{1}(k)\right)}{\overline{\mathcal{L}}_{1}} P-\frac{i}{3} P \bar{P}+\frac{i}{9}\left(\frac{\overline{\mathcal{L}}_{1}\left(\overline{\mathcal{L}}_{1}(k)\right)}{\overline{\mathcal{L}}_{1}(k)}-\bar{P}\right)\left(\frac{\mathcal{L}_{1}\left(\mathcal{L}_{1}(\bar{k})\right)}{\mathcal{L}_{1}(\overline{\bar{k}})}-P\right) .
\end{aligned}
$$

Secondly

$$
\begin{aligned}
{\left[\rho_{0} \wedge \zeta_{0}^{\prime}\right]\left\{d \kappa_{0}^{\prime}\right\}=} & -\frac{\mathcal{T}(k)}{\overline{\mathcal{L}}_{1}(k)}-\frac{i}{3} \frac{\mathcal{K}\left(\overline{\mathcal{L}}_{1}\left(\overline{\mathcal{L}}_{1}(k)\right)\right)}{\overline{\mathcal{L}}_{1}(k)^{2}}+\frac{i}{3} \frac{\mathcal{K}\left(\overline{\mathcal{L}}_{1}(k)\right) \overline{\mathcal{L}}_{1}\left(\overline{\mathcal{L}}_{1}(k)\right)}{\overline{\mathcal{L}}_{1}(k)^{3}} \\
& +\frac{i}{3} \frac{\mathcal{\mathcal { K } ( \overline { P } )}}{\overline{\overline{\mathcal{L}}_{1}(k)}}-\frac{i}{3} \frac{\mathcal{L}_{1}\left(\mathcal{L}_{1}(\bar{k})\right)}{\mathcal{L}_{1}(\bar{k})}+\frac{i}{3} P
\end{aligned}
$$

but here, we must still replace the boxed term using Lemma 2.5

$$
\begin{aligned}
{\left[\rho_{0} \wedge \zeta_{0}^{\prime}\right]\left\{d \kappa_{0}^{\prime}\right\}=} & -\frac{\mathcal{T}(k)}{\overline{\mathcal{L}}_{1}(k)}-\frac{i}{3} \frac{\mathcal{K}\left(\overline{\mathcal{L}}_{1}\left(\overline{\mathcal{L}}_{1}(k)\right)\right)}{\overline{\mathcal{L}}_{1}(k)^{2}}+\frac{i}{3} \frac{\mathcal{K}\left(\overline{\mathcal{L}}_{1}(k)\right) \overline{\mathcal{L}}_{1}\left(\overline{\mathcal{L}}_{1}(k)\right)}{\overline{\mathcal{L}}_{1}(k)^{3}} \\
& -\frac{i}{3} P-\frac{i}{3} \frac{\overline{\mathcal{L}}_{1}\left(\mathcal{L}_{1}(k)\right)}{\overline{\mathcal{L}}_{1}(k)}+\frac{1}{3} \frac{\mathcal{T}(k)}{\overline{\mathcal{L}}_{1}(k)}-\frac{i}{3} \frac{\mathcal{L}_{1}\left(\mathcal{L}_{1}(\bar{k})\right)}{\mathcal{L}_{1}(\bar{k})}+\frac{i}{3} P .
\end{aligned}
$$

A pair cancellation makes the obtained expression match precisely with what Proposition 7.2 stated, after some permutation of terms.

The third replacement conducts directly to the stated result

$$
\begin{aligned}
{\left[\rho_{0} \wedge \bar{\kappa}_{0}^{\prime}\right]\left\{d \kappa_{0}^{\prime}\right\}=} & -\frac{i}{3} \frac{\overline{\mathcal{L}}_{1}\left(\overline{\mathcal{L}}_{1}\left(\overline{\mathcal{L}}_{1}(k)\right)\right)}{\overline{\mathcal{L}}_{1}(k)}+\frac{i}{3} \frac{\overline{\mathcal{L}}_{1}\left(\overline{\mathcal{L}}_{1}(k)\right)^{2}}{\overline{\mathcal{L}}_{1}(k)^{2}}+\frac{i}{3} \overline{\mathcal{L}}_{1}(\bar{P}) \\
& +\frac{i}{3} \frac{\overline{\mathcal{L}}_{1}\left(\overline{\mathcal{L}}_{1}(k)\right)}{\overline{\mathcal{L}}_{1}(k)} \overline{\boldsymbol{P}}-\frac{i}{3} \bar{P} \bar{P} \\
& +\frac{i}{9} \frac{\overline{\mathcal{L}}_{1}\left(\overline{\mathcal{L}}_{1}(k)\right)^{2}}{\overline{\mathcal{L}}_{1}(k)^{2}}-\frac{2 i}{9} \frac{\overline{\mathcal{L}}_{1}\left(\overline{\mathcal{L}}_{1}(k)\right)}{\overline{\mathcal{L}}_{1}(k)} \bar{P}+\frac{i}{9} \bar{P} \bar{P}
\end{aligned}
$$


while the fourth (last) brings an identically zero result

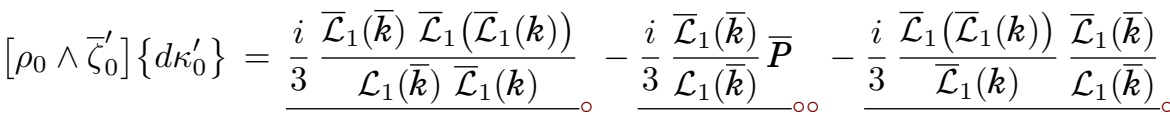

$$
\begin{aligned}
& +\frac{i}{3} \bar{P} \frac{\overline{\mathcal{L}}_{1}(\bar{k})}{\mathcal{L}_{1}(\bar{k})}
\end{aligned}
$$

\section{THIRD LOOP: REDUCTION OF THE GROUP PARAMETER d}

After normalization of the group parameter b from (6.7), we have a new reduced group $G^{6}$ of real dimension 6 , and the lifted coframe is

$$
\left(\begin{array}{l}
\rho \\
\kappa \\
\zeta
\end{array}\right):=\left(\begin{array}{ccc}
\mathrm{c} \overline{\mathrm{c}} & 0 & 0 \\
-i \overline{\mathrm{c} e} & \mathrm{c} & 0 \\
\mathrm{~d} & \mathrm{e} & \overline{\mathrm{c}}
\end{array}\right)\left(\begin{array}{c}
\rho_{0} \\
\kappa_{0}^{\prime} \\
\zeta_{0}^{\prime}
\end{array}\right) \quad \Longleftrightarrow \quad\left\{\begin{array}{l}
\rho:=\mathrm{c} \overline{\mathrm{c}} \rho_{0}, \\
\kappa:=-i \overline{\mathrm{c} e} \rho_{0}+\mathrm{c} \kappa_{0}^{\prime}, \\
\zeta:=\mathrm{d} \rho_{0}+\mathrm{e} \kappa_{0}^{\prime}+\frac{\mathrm{c}}{\overline{\mathrm{c}}} \zeta_{0}^{\prime},
\end{array}\right.
$$

with inverse formulas

$$
\begin{aligned}
\rho_{0} & =\frac{1}{\mathrm{cc}} \rho, \\
\kappa_{0}^{\prime} & =i \frac{\mathrm{e}}{\mathrm{cc}} \rho+\frac{1}{\mathrm{c}} \kappa, \\
\zeta_{0}^{\prime} & =\left(-i \frac{\overline{\mathrm{cee}}}{\mathrm{ccc}}-\frac{\mathrm{d}}{\mathrm{cc}}\right) \rho-\frac{\overline{\mathrm{ce}}}{\mathrm{cc}} \kappa+\frac{\overline{\mathrm{c}}}{\mathrm{c}} \zeta .
\end{aligned}
$$

The Maurer-Cartan matrix becomes

$$
\begin{aligned}
d g \cdot g^{-1} & =\left(\begin{array}{ccc}
\bar{c} d \mathrm{c}+\mathrm{c} d \overline{\bar{c}} & 0 & 0 \\
-i \mathrm{e} d \overline{\mathrm{c}}-i \overline{\mathrm{c}} d \mathrm{e} & d \mathrm{c} & 0 \\
d \mathrm{~d} & d \mathrm{e} & \frac{d c}{\overline{\bar{c}}}-\frac{\mathrm{c} d \overline{\bar{c}}}{\bar{c} \mathrm{c}}
\end{array}\right)\left(\begin{array}{ccc}
\frac{1}{\bar{c}} & 0 & 0 \\
i \frac{\mathrm{e}}{\mathrm{cc}} & \frac{1}{\mathrm{c}} & 0 \\
-i \frac{\mathrm{ce}}{\mathrm{ccc}}-\frac{\mathrm{d}}{\mathrm{cc}} & -\frac{\bar{c} \mathrm{e}}{\mathrm{cc}} & \frac{\bar{c}}{\bar{c}}
\end{array}\right) \\
& =:\left(\begin{array}{ccc}
\alpha+\bar{\alpha} & 0 & 0 \\
\beta & \alpha & 0 \\
\gamma & i \beta & \alpha-\bar{\alpha}
\end{array}\right),
\end{aligned}
$$

in terms of the group-invariant 1 -forms

$$
\begin{aligned}
& \alpha:=\frac{d \mathrm{c}}{\mathrm{c}}, \\
& \beta:=i \frac{\mathrm{e} d \mathrm{c}}{\mathrm{cc}}-i \frac{\mathrm{e} d \overline{\mathrm{c}}}{\mathrm{c} \overline{\mathrm{c}}}-i \frac{d \mathrm{e}}{\mathrm{c}}, \\
& \gamma:=\left(\frac{\mathrm{cd}+i \overline{\mathrm{c} e e}}{\mathrm{cc} \bar{c}}\right)\left(-\frac{d \mathrm{c}}{\mathrm{c}}+\frac{d \overline{\mathrm{c}}}{\overline{\mathrm{c}}}\right)+\frac{d \mathrm{~d}}{\mathrm{c} \overline{\mathrm{c}}}+i \frac{\mathrm{e} d \mathrm{e}}{\mathrm{cc}} .
\end{aligned}
$$

Now, if we exterior-differentiate the lifted coframe on the product manifold equipped with coordinates

$$
\left(z_{1}, z_{2}, \bar{z}_{1}, \bar{z}_{2}, v\right) \times(\mathrm{c}, \overline{\mathrm{c}}, \mathrm{d}, \overline{\mathrm{d}}, \mathrm{e}, \overline{\mathrm{e}}) \in M^{5} \times G^{6},
$$


after some computations, we may come to structure equations of the abstract shape

$$
\begin{aligned}
d \rho= & (\alpha+\bar{\alpha}) \wedge \rho \\
& +R^{1} \rho \wedge \kappa+R^{2} \rho \wedge \zeta+\bar{R}^{1} \rho \wedge \bar{\kappa}+\bar{R}^{2} \rho \wedge \bar{\zeta}+i \kappa \wedge \bar{\kappa}, \\
d \kappa= & \beta \wedge \rho+\alpha \wedge \kappa \\
& +K^{1} \rho \wedge \kappa+K^{2} \rho \wedge \zeta+K^{3} \rho \wedge \bar{\kappa}+K^{4} \rho \wedge \bar{\zeta} \\
& +K^{5} \kappa \wedge \zeta+K^{6} \kappa \wedge \bar{\kappa}+\zeta \wedge \bar{\kappa}, \\
d \zeta= & \gamma \wedge \rho+i \beta \wedge \kappa+(\alpha-\bar{\alpha}) \wedge \zeta \\
& +Z^{1} \rho \wedge \kappa+Z^{2} \rho \wedge \zeta+Z^{3} \rho \wedge \bar{\kappa}+Z^{4} \rho \wedge \bar{\zeta} \\
& +Z^{5} \kappa \wedge \zeta+Z^{6} \kappa \wedge \bar{\kappa}+Z^{7} \kappa \wedge \bar{\zeta}+Z^{8} \zeta \wedge \bar{\kappa}+Z^{9} \zeta \wedge \bar{\zeta} .
\end{aligned}
$$

Before really computing explicitly some of these torsion coefficients, let us examine what are the absorption equations. For this, we replace

$$
\begin{aligned}
& \alpha=: \alpha^{\prime}+a_{1} \rho+a_{2} \kappa+a_{3} \zeta+a_{4} \bar{\kappa}+a_{5} \bar{\zeta}, \\
& \beta=: \beta^{\prime}+b_{1} \rho+b_{2} \kappa+b_{3} \zeta+b_{4} \bar{\kappa}+b_{5} \bar{\zeta}, \\
& \gamma=: \gamma^{\prime}+c_{1} \rho+c_{2} \kappa+c_{3} \zeta+c_{4} \bar{\kappa}+c_{5} \bar{\zeta} .
\end{aligned}
$$

A moment of reflection convinces that the result for $d \rho$ is the same as in the proof of Lemma 6.4:

$$
\begin{aligned}
d \rho= & \left(\alpha^{\prime}+\bar{\alpha}^{\prime}\right) \wedge \rho \\
& +\rho \wedge \kappa\left(R^{1}-a_{2}-\bar{a}_{4}\right)+\rho \wedge \zeta\left(R^{2}-a_{3}-\bar{a}_{5}\right)+\rho \wedge \bar{\kappa}\left(\bar{R}^{1}-a_{4}-\bar{a}_{2}\right) \\
& +\rho \wedge \bar{\zeta}\left(\bar{R}^{2}-a_{5}-\bar{a}_{3}\right)+i \kappa \wedge \bar{\kappa} .
\end{aligned}
$$

Similarly, $d \kappa$ is unchanged

$$
\begin{aligned}
d \kappa= & \beta^{\prime} \wedge \rho+\alpha^{\prime} \wedge \kappa \\
& +\rho \wedge \kappa\left(K^{1}+a_{1}-b_{2}\right)+\rho \wedge \zeta\left(K^{2}-b_{3}\right)+\rho \wedge \bar{\kappa}\left(K^{3}-b_{4}\right)+\rho \wedge \bar{\zeta}\left(K^{4}-b_{5}\right) \\
& +\kappa \wedge \zeta\left(K^{5}-a_{3}\right)+\kappa \wedge \bar{\kappa}\left(K^{6}-a_{4}\right)+\kappa \wedge \bar{\zeta}\left(-a_{5}\right)+\zeta \wedge \bar{\kappa} .
\end{aligned}
$$

However, for $d \zeta$, we have to compute

$$
\begin{aligned}
\gamma \wedge \rho+i \beta \wedge \kappa+(\alpha-\bar{\alpha}) \wedge \zeta= & \gamma^{\prime} \wedge \rho+0+c_{2} \kappa \wedge \rho+c_{3} \zeta \wedge \rho+c_{4} \bar{\kappa} \wedge \rho+c_{5} \bar{\zeta} \wedge \rho \\
& +i \beta^{\prime} \wedge \kappa+i b_{1} \rho \wedge \kappa+0+i b_{3} \zeta \wedge \kappa+i b_{4} \bar{\kappa} \wedge \kappa+i b_{5} \bar{\zeta} \wedge \kappa \\
& +\alpha^{\prime} \wedge \zeta+a_{1} \rho \wedge \zeta+a_{2} \kappa \wedge \zeta+0+a_{4} \bar{\kappa} \wedge \zeta+a_{5} \bar{\zeta} \wedge \zeta \\
& -\bar{\alpha}^{\prime} \wedge \zeta-\bar{a}_{1} \rho \wedge \zeta-\bar{a}_{2} \bar{\kappa} \wedge \zeta-\bar{a}_{3} \bar{\zeta} \wedge \zeta-\bar{a}_{4} \kappa \wedge \zeta-0
\end{aligned}
$$

and we get

$$
\begin{aligned}
d \zeta= & \gamma^{\prime} \wedge \rho+i \beta^{\prime} \wedge \kappa+\left(\alpha^{\prime}-\bar{\alpha}^{\prime}\right) \wedge \zeta \\
& +\rho \wedge \kappa\left(Z^{1}+i b_{1}-c_{2}\right)+\rho \wedge \zeta\left(Z^{2}-c_{3}+a_{1}-\bar{a}_{1}\right)+\rho \wedge \bar{\kappa}\left(Z^{3}-c_{4}\right)+\rho \wedge \bar{\zeta}\left(Z^{4}-c_{5}\right) \\
& +\kappa \wedge \zeta\left(Z^{5}-i b_{3}+a_{2}-\bar{a}_{4}\right)+\kappa \wedge \bar{\kappa}\left(Z^{6}-i b_{4}\right)+\kappa \wedge \bar{\zeta}\left(Z^{7}-i b_{5}\right) \\
& +\zeta \wedge \bar{\kappa}\left(Z^{8}-a_{4}+\bar{a}_{2}\right)+\zeta \wedge \bar{\zeta}\left(Z^{9}-a_{5}+\bar{a}_{3}\right) .
\end{aligned}
$$


Lemma 8.3. Here is an essential linear combination of torsion terms

$$
i K^{3}-Z^{6} \text {. }
$$

Proof. Indeed,

$$
\begin{aligned}
K^{3 \prime} & =K^{3}-b_{4}, \\
Z^{6 \prime} & =Z^{6}-i b_{4},
\end{aligned}
$$

whence

$$
i K^{3 \prime}-Z^{6 \prime}=i K^{3}-Z^{6} .
$$

Proposition 8.4. Their explicit expressions are

$$
\begin{aligned}
K^{3}= & -\frac{\mathrm{d}}{\mathrm{c} \overline{\mathrm{C}}}+\frac{\mathrm{e}}{\mathrm{c} \overline{\mathrm{C}}}\left(-2 i \frac{\overline{\mathcal{L}}_{1}\left(\overline{\mathcal{L}}_{1}(k)\right)}{\overline{\mathcal{L}}_{1}(k)}-\frac{i}{3} \bar{P}\right)-i \frac{\mathrm{e}}{\overline{\mathrm{cC}}} \frac{\overline{\mathcal{L}}_{1}(k)}{\mathcal{L}_{1}(\bar{k})} \\
& +\frac{1}{\mathrm{c} \overline{\mathrm{C}}}\left(-\frac{i}{3} \frac{\overline{\mathcal{L}}_{1}\left(\overline{\mathcal{L}}_{1}\left(\overline{\mathcal{L}}_{1}(k)\right)\right)}{\overline{\mathcal{L}}_{1}(k)}+\frac{4 i}{9} \frac{\overline{\mathcal{L}}_{1}\left(\overline{\mathcal{L}}_{1}(k)\right)^{2}}{\overline{\mathcal{L}}_{1}(k)^{2}}\right. \\
& \left.+\frac{i}{9} \frac{\overline{\mathcal{L}}_{1}\left(\overline{\mathcal{L}}_{1}(k)\right)}{\overline{\mathcal{L}}_{1}(k)} \bar{P}+\frac{i}{3} \overline{\mathcal{L}}_{1}(\bar{P})-\frac{2 i}{9} \bar{P} \bar{P}\right), \\
Z^{6}= & i \frac{\mathrm{d}}{\mathrm{c} \overline{\mathrm{C}}}-\frac{\mathrm{ee}}{\mathrm{cc}}+\frac{\mathrm{e}}{\mathrm{c} \overline{\mathrm{C}}}\left(\frac{1}{3} \bar{P}+\frac{2}{3} \frac{\overline{\mathcal{L}}_{1}\left(\overline{\mathcal{L}}_{1}(k)\right)}{\overline{\mathcal{L}}_{1}(k)}\right)+\frac{\overline{\mathrm{ee}}}{\overline{\mathrm{cc}}} \frac{\overline{\mathcal{L}}_{1}(\bar{k})}{\mathcal{L}_{1}(\bar{k})} .
\end{aligned}
$$

Proof. We start by differentiating (8.1), finalizing directly the Maurer-Cartan part, thanks to the Maurer-Cartan matrix shown above, and setting aside $d \rho$ for the moment

$$
\begin{aligned}
d \kappa= & \beta \wedge \rho+\alpha \wedge \kappa \\
& -i \overline{\mathrm{ce}} d \rho_{0}+\mathrm{c} d \kappa_{0}^{\prime}, \\
d \zeta= & \gamma \wedge \rho+i \beta \wedge \kappa+(\alpha-\bar{\alpha}) \wedge \zeta \\
& +\mathrm{d} d \rho_{0}+\mathrm{e} d \kappa_{0}^{\prime}+\frac{\mathrm{c}}{\overline{\mathrm{c}}} d \zeta_{0}^{\prime} .
\end{aligned}
$$

So we have to compute first

$$
\begin{aligned}
K^{3} & =[\rho \wedge \bar{\kappa}]\{d \kappa\} \\
& =-i \overline{\mathrm{ce}}[\rho \wedge \bar{\kappa}]\left\{d \rho_{0}\right\}+\mathrm{c}[\rho \wedge \bar{\kappa}]\left\{d \kappa_{0}^{\prime}\right\} .
\end{aligned}
$$

The first term is, by (7.1), using the inversion formulas (8.2)

$$
\begin{aligned}
{[\rho \wedge \bar{\kappa}]\left\{d \rho_{0}\right\} } & =[\rho \wedge \bar{\kappa}]\left\{0+0+\bar{R}_{0}^{1 \prime}\left(\frac{1}{\mathrm{c} \overline{\mathrm{C}}}\right) \wedge\left(\frac{1}{\overline{\mathrm{C}}} \bar{\kappa}\right)+\bar{R}_{0}^{2 \prime}\left(\frac{1}{\mathrm{c} \overline{\mathrm{C}}}\right) \wedge\left(-\frac{\mathrm{ce}}{\overline{\mathrm{CC}}} \bar{\kappa}\right)+i\left(i \frac{\mathrm{e}}{\mathrm{cC}} \rho\right) \wedge\left(\frac{1}{\overline{\mathrm{C}}} \bar{\kappa}\right)\right\} \\
& =\frac{1}{\mathrm{c} \overline{\mathrm{CC}}} \bar{R}_{0}^{1 \prime}-\frac{\overline{\mathrm{e}}}{\overline{\mathrm{CCC}}} \bar{R}_{0}^{2 \prime}-\frac{\mathrm{e}}{\mathrm{cc} \overline{\mathrm{C}}} .
\end{aligned}
$$

Similarly

$$
\begin{aligned}
{[\rho \wedge \bar{\kappa}]\left\{d \kappa_{0}^{\prime}\right\}=} & {[\rho \wedge \bar{\kappa}]\left\{0+0+K_{0}^{3 \prime}\left(\frac{1}{\mathrm{c} \overline{\mathrm{C}}} \rho\right) \wedge\left(\frac{1}{\overline{\mathrm{c}}} \bar{\kappa}\right)\right.} \\
& \left.+0+K_{0}^{6 \prime}\left(i \frac{\mathrm{e}}{\mathrm{cc}} \rho\right) \wedge\left(\frac{1}{\overline{\mathrm{c}}} \bar{\kappa}\right)+\left(\left(-i \frac{\overline{\mathrm{cee}}}{\mathrm{ccc}}-\frac{\mathrm{d}}{\mathrm{cc}}\right) \rho\right) \wedge\left(\frac{1}{\overline{\mathrm{c}}} \bar{\kappa}\right)\right\} \\
= & \frac{1}{\mathrm{c} \overline{\mathrm{CC}}} K_{0}^{3 \prime}+i \frac{\mathrm{e}}{\mathrm{cc} \overline{\mathrm{C}}} K_{0}^{6 \prime}-i \frac{\mathrm{ee}}{\mathrm{ccc}}-\frac{\mathrm{d}}{\mathrm{cc} \overline{\mathrm{c}}} .
\end{aligned}
$$


Hence

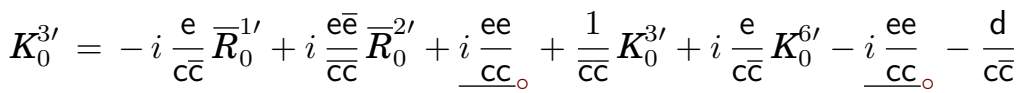

$$
\begin{aligned}
& =-\frac{\mathrm{d}}{\mathrm{c} \overline{\mathrm{C}}}+\frac{\mathrm{e}}{\mathrm{c} \overline{\mathrm{C}}}\left(-\frac{i}{3} \frac{\overline{\mathcal{L}}_{1}\left(\overline{\mathcal{L}}_{1}(k)\right)}{\overline{\mathcal{L}}_{1}(k)}-\frac{2 i}{3} \bar{P}-\frac{i}{3} \overline{\mathcal{L}}_{1}\left(\overline{\mathcal{L}}_{1}(k)\right)+\frac{i}{3} \bar{p}\right)-i \frac{\mathrm{e} \overline{\overline{\mathrm{e}}}}{\overline{\mathrm{CC}}} \frac{\overline{\mathcal{L}}_{1}(\bar{k})}{\mathcal{L}_{1}(\bar{k})}+\frac{1}{\overline{\mathrm{CC}}} K_{0}^{3 \prime} .
\end{aligned}
$$

Replacing this last term $K_{0}^{3 \prime}$ by its value from Proposition 7.2, we reach the stated explicit expression of $K^{3}$. Next

$$
\begin{aligned}
Z^{6} & =[\kappa \wedge \bar{\kappa}]\{d \zeta\} \\
& =\mathrm{d}[\kappa \wedge \bar{\kappa}]\left\{d \rho_{0}\right\}+\mathrm{e}[\kappa \wedge \bar{\kappa}]\left\{d \kappa_{0}^{\prime}\right\}+\frac{\mathrm{c}}{\overline{\mathrm{c}}}[\kappa \wedge \bar{\kappa}]\left\{d \zeta_{0}^{\prime}\right\} .
\end{aligned}
$$

Separately

$$
\begin{aligned}
{[\kappa \wedge \bar{\kappa}]\left\{\mathrm{d} d \rho_{0}\right\} } & =0+0+0+0+\mathrm{d} i \frac{1}{\mathrm{c} \overline{\mathrm{c}}}=i \frac{\mathrm{d}}{\mathrm{c} \overline{\mathrm{c}}} \\
{[\kappa \wedge \bar{\kappa}]\left\{\mathrm{e} d \kappa_{0}^{\prime}\right\} } & =0+0+0+0+\mathrm{e} K_{0}^{6 \prime} \frac{1}{\mathrm{c} \overline{\mathrm{c}}}+\mathrm{e}\left(-\frac{\overline{\mathrm{ce}}}{\mathrm{cc}}\right) \frac{1}{\overline{\mathrm{c}}}=\frac{\mathrm{e}}{\mathrm{c \overline {c }}} K_{0}^{6 \prime}-\frac{\mathrm{ee}}{\mathrm{cc}}, \\
{[\kappa \wedge \bar{\kappa}]\left\{\frac{\mathrm{c}}{\overline{\mathrm{c}}} d \zeta_{0}^{\prime}\right\} } & =0+0+\frac{\mathrm{c}}{\overline{\mathrm{c}}} Z_{0}^{8 \prime}\left(-\frac{\overline{\mathrm{ce}}}{\mathrm{cc}}\right)\left(\frac{1}{\overline{\mathrm{c}}}\right)+\frac{\mathrm{c}}{\overline{\mathrm{c}}} Z_{0}^{9 \prime}\left(-\frac{\overline{\mathrm{ce}}}{\mathrm{cc}}\right)\left(-\frac{\mathrm{c} \overline{\mathrm{e}}}{\overline{\mathrm{cc}}}\right) \\
& =-\frac{\mathrm{e}}{\mathrm{c} \overline{\mathrm{c}}} Z_{0}^{8 \prime}+\frac{\mathrm{ee}}{\overline{\mathrm{cc}}} Z_{0}^{9 \prime},
\end{aligned}
$$

hence summing and inserting the explicit expressions from Proposition 7.2, we conclude

$$
\begin{aligned}
Z^{6} & =i \frac{\mathrm{d}}{\mathrm{c} \overline{\mathrm{C}}}+\frac{\mathrm{e}}{\mathrm{c} \overline{\mathrm{C}}} K_{0}^{6 \prime}-\frac{\mathrm{ee}}{\mathrm{cc}}-\frac{\mathrm{e}}{\mathrm{c} \overline{\mathrm{C}}} Z_{0}^{8 \prime}+\frac{\mathrm{e} \overline{\mathrm{e}}}{\overline{\mathrm{CC}}} Z_{0}^{9 \prime} \\
& =i \frac{\mathrm{d}}{\mathrm{c} \overline{\mathrm{C}}}-\frac{\mathrm{ee}}{\mathrm{cc}}+\frac{\mathrm{e}}{\mathrm{c} \overline{\mathrm{C}}}\left(\frac{1}{3} \bar{P}+\frac{2}{3} \frac{\overline{\mathcal{L}}_{1}\left(\overline{\mathcal{L}}_{1}(k)\right)}{\overline{\mathcal{L}}_{1}(k)}\right)+\frac{\overline{\mathrm{ee}}}{\overline{\overline{\mathrm{CC}}}} \frac{\overline{\mathcal{L}}_{1}(\bar{k})}{\mathcal{L}_{1}(\bar{k})} .
\end{aligned}
$$

Once we have reached the explicit expressions of both $K^{3}$ and $Z^{6}$, when we perform the essential combination $i K^{3}-Z^{6}$, we see that both the coefficients of $\frac{\mathrm{e}}{\bar{c} \bar{c}}$ and of $\frac{\mathrm{e} \overline{\mathrm{e}}}{\overline{c c}}$ disappear, and it remains

$$
\begin{aligned}
i K^{3}-Z^{6}= & -2 i \frac{\mathrm{d}}{\mathrm{c} \overline{\mathrm{c}}}+\frac{\mathrm{ee}}{\mathrm{cc}}+i \frac{1}{\mathrm{c} \overline{\mathrm{C}}} K_{0}^{3 \prime} \\
= & -2 i \frac{\mathrm{d}}{\mathrm{c} \overline{\mathrm{c}}}+\frac{\mathrm{ee}}{\mathrm{cc}} \\
& +\frac{1}{\mathrm{c} \overline{\mathrm{c}}}(\underbrace{\frac{1}{3} \frac{\overline{\mathcal{L}}_{1}\left(\overline{\mathcal{L}}_{1}\left(\overline{\mathcal{L}}_{1}(k)\right)\right)}{\overline{\mathcal{L}}_{1}(k)}-\frac{4}{9} \frac{\overline{\mathcal{L}}_{1}\left(\overline{\mathcal{L}}_{1}(k)\right)^{2}}{\overline{\mathcal{L}}_{1}(k)^{2}}-\frac{1}{9} \frac{\overline{\mathcal{L}}_{1}\left(\overline{\mathcal{L}}_{1}(k)\right)}{\overline{\mathcal{L}}_{1}(k)} \bar{P}-\frac{1}{3} \overline{\mathcal{L}}_{1}(\bar{P})+\frac{2}{9} \bar{P} \bar{P}}_{=:-2 H_{0}}) .
\end{aligned}
$$

We introduce, as is underbraced

$$
H_{0}:=-\frac{1}{6} \frac{\overline{\mathcal{L}}_{1}\left(\overline{\mathcal{L}}_{1}\left(\overline{\mathcal{L}}_{1}(k)\right)\right)}{\overline{\mathcal{L}}_{1}(k)}+\frac{2}{9} \frac{\overline{\mathcal{L}}_{1}\left(\overline{\mathcal{L}}_{1}(k)\right)^{2}}{\overline{\mathcal{L}}_{1}(k)^{2}}+\frac{1}{18} \frac{\overline{\mathcal{L}}_{1}\left(\overline{\mathcal{L}}_{1}(k)\right)}{\overline{\mathcal{L}}_{1}(k)} \bar{P}+\frac{1}{6} \overline{\mathcal{L}}_{1}(\bar{P})-\frac{1}{9} \bar{P} \bar{P},
$$

a function which coincides with Pocchiola's function $H$. Then by means of the invariant condition

$$
0=i K^{3}-Z^{6}
$$


we reach a convenient normalization of the group parameter

$$
\begin{aligned}
\mathrm{d} & :=-\frac{i}{2} \frac{\overline{\mathrm{cee}}}{\mathrm{c}}+i \frac{\mathrm{c}}{\overline{\mathrm{c}}} H_{0} \\
& =-\frac{i}{2} \frac{\overline{\mathrm{cee}}}{\mathrm{c}}+i \frac{\mathrm{c}}{\overline{\mathrm{c}}}\left(-\frac{1}{6} \frac{\overline{\mathcal{L}}_{1}\left(\overline{\mathcal{L}}_{1}\left(\overline{\mathcal{L}}_{1}(k)\right)\right)}{\overline{\mathcal{L}}_{1}(k)}+\frac{2}{9} \frac{\overline{\mathcal{L}}_{1}\left(\overline{\mathcal{L}}_{1}(k)\right)^{2}}{\overline{\mathcal{L}}_{1}(k)^{2}}+\frac{1}{18} \frac{\overline{\mathcal{L}}_{1}\left(\overline{\mathcal{L}}_{1}(k)\right)}{\overline{\mathcal{L}}_{1}(k)} \bar{P}+\frac{1}{6} \overline{\mathcal{L}}_{1}(\bar{P})-\frac{1}{9} \bar{P} \bar{P}\right) .
\end{aligned}
$$

Before we really perform this normalization of the group parameter $d$, let us point out that some other invariant relations between torsion coefficients appear. In fact, we see above that:

$$
\begin{aligned}
i K^{4 \prime} & =i K^{4}-i b_{5}, \\
Z^{7 \prime} & =Z^{7}-i b_{5},
\end{aligned}
$$

whence

$$
i K^{4 \prime}-Z^{7 \prime}=i K^{4}-Z^{7} \text {. }
$$

However, the next lemma shows that no group parameter can be normalized so.

Lemma 8.5. Their explicit expressions are

$$
i K^{4}=Z^{7}=-\frac{\mathrm{e}}{\mathrm{c}} \frac{\overline{\mathcal{L}}_{1}(\bar{k})}{\mathcal{L}_{1}(\bar{k})} .
$$

Proof. Indeed, by (7.1), replacing $\bar{R}_{0}^{2 \prime}$ from Proposition 7.2, we can compute using (8.2)

$$
\begin{aligned}
K^{4} & =[\rho \wedge \bar{\zeta}]\left\{-i \overline{\mathrm{ce}} d \rho_{0}+\mathrm{c} d \kappa_{0}^{\prime}\right\} \\
& =-i \overline{\mathrm{ce}}[\rho \wedge \bar{\zeta}]\left\{d \rho_{0}\right\}+\mathrm{c}[\rho \wedge \bar{\zeta}]\left\{d \kappa_{0}^{\prime}\right\} \\
& =-i \overline{\mathrm{ce}}\left(0+0+0+\bar{R}_{0}^{2 \prime}\left(\frac{1}{\mathrm{c} \overline{\mathrm{c}}}\right)\left(\frac{\mathrm{c}}{\overline{\mathrm{c}}}\right)\right)+\mathrm{c} \cdot 0 \\
& =-i \frac{\mathrm{e}}{\overline{\mathrm{c}}}\left(-\frac{\overline{\mathcal{L}}_{1}(\bar{k})}{\overline{\mathcal{L}}_{1}(k)}\right)
\end{aligned}
$$

and similarly

$$
\begin{aligned}
Z^{7} & =[\kappa \wedge \bar{\zeta}]\left\{\mathrm{d} d \rho_{0}+\mathrm{e} d \kappa_{0}^{\prime}+\frac{\mathrm{c}}{\overline{\mathrm{c}}} d \zeta_{0}^{\prime}\right\} \\
& =\mathrm{d}[\kappa \wedge \bar{\zeta}]\left\{d \rho_{0}\right\}+\mathrm{e}[\kappa \wedge \bar{\zeta}]\left\{d \kappa_{0}^{\prime}\right\}+\frac{\mathrm{c}}{\overline{\mathrm{c}}}[\kappa \wedge \bar{\zeta}]\left\{d \zeta_{0}^{\prime}\right\} \\
& =0+0+\frac{\mathrm{c}}{\overline{\overline{\mathrm{c}}}}\left(0+0+0+Z_{0}^{9 \prime}\left(-\frac{\overline{\mathrm{c} e}}{\mathrm{cc}}\right)\left(\frac{\mathrm{c}}{\overline{\mathrm{c}}}\right)\right) \\
& =-\frac{\mathrm{e}}{\overline{\mathrm{c}}} \frac{\overline{\mathcal{L}}_{1}(\bar{k})}{\overline{\mathcal{L}}_{1}(k)} .
\end{aligned}
$$

Another invariant torsion combination is the following.

Lemma 8.6. Here is an essential linear combination of torsion terms

$$
-i K^{2}+Z^{5}-\bar{Z}^{8}
$$

Proof. A glance at what precedes shows

$$
\begin{aligned}
K^{2 \prime} & =K^{2}-b_{3}, \\
Z^{5 \prime} & =Z^{5}-i b_{3}+a_{2}-\bar{a}_{4}, \\
Z^{8 \prime} & =Z^{8}-a_{4}+\bar{a}_{2},
\end{aligned}
$$


whence indeed

$$
-i K^{2 \prime}+Z^{5 \prime}-\bar{Z}^{8 \prime}=-i K^{2}+Z^{5}-\bar{Z}^{8}
$$

Lemma 8.7. Their explicit expressions are:

$$
\begin{aligned}
K^{2}= & i \frac{\overline{\mathrm{e}}}{\mathrm{c}}+\frac{1}{\mathrm{c}}\left(-\frac{i}{3} \frac{\mathcal{K}\left(\overline{\mathcal{L}}_{1}\left(\overline{\mathcal{L}}_{1}(k)\right)\right)}{\overline{\mathcal{L}}_{1}(k)^{2}}+\frac{i}{3} \frac{\mathcal{K}\left(\overline{\mathcal{L}}_{1}(k)\right) \overline{\mathcal{L}}_{1}\left(\overline{\mathcal{L}}_{1}(k)\right)}{\overline{\mathcal{L}}_{1}(k)^{3}}\right. \\
& \left.-\frac{i}{3} \frac{\mathcal{L}_{1}\left(\mathcal{L}_{1}(\bar{k})\right)}{\mathcal{L}_{1}(\bar{k})}-\frac{i}{3} \frac{\overline{\mathcal{L}}_{1}\left(\mathcal{L}_{1}(k)\right)}{\overline{\mathcal{L}}_{1}(k)}-\frac{2}{3} \frac{\mathcal{T}(k)}{\overline{\mathcal{L}}_{1}(k)}\right), \\
Z^{5}= & \frac{1}{\mathrm{c}} \frac{\mathcal{L}_{1}\left(\overline{\mathcal{L}}_{1}(k)\right)}{\overline{\mathcal{L}}_{1}(k)}-\frac{\overline{\mathrm{ce}}}{\mathrm{cc}} \frac{\mathcal{L}_{1}(\bar{k})}{\overline{\mathcal{L}}_{1}(k)} \\
Z^{8}= & \frac{\mathrm{e}}{\mathrm{c}}-\frac{1}{\overline{\mathrm{c}}} \frac{\overline{\mathcal{L}}_{1}\left(\overline{\mathcal{L}}_{1}(k)\right)}{\overline{\mathcal{L}}_{1}(k)}-\frac{\mathrm{ce}}{\overline{\mathrm{cc}}} \frac{\overline{\mathcal{L}}_{1}(k)}{\mathcal{L}_{1}(\bar{k})} .
\end{aligned}
$$

Proof. Recall

$$
\begin{aligned}
& d \rho=(\alpha+\bar{\alpha}) \wedge \rho+\mathrm{c} \overline{\mathrm{c}} d \rho_{0} \\
& d \kappa=\beta \wedge \rho+\alpha \wedge \kappa-i \overline{\mathrm{c} e} d \rho_{0}+\mathrm{c} d \kappa_{0}^{\prime}, \\
& d \zeta=\gamma \wedge \rho+i \beta \wedge \kappa+(\alpha-\bar{\alpha}) \wedge \rho+\mathrm{d} d \rho_{0}+\mathrm{e} d \kappa_{0}^{\prime}+\frac{\mathrm{c}}{\overline{\mathrm{c}}} d \zeta_{0}^{\prime}
\end{aligned}
$$

hence

$$
K^{2}=[\rho \wedge \zeta]\left\{-i \overline{\mathrm{ce}} d \rho_{0}+\mathrm{c} d \kappa_{0}^{\prime}\right\}
$$

Visually

$$
\begin{aligned}
{[\rho \wedge \zeta]\left\{d \rho_{0}\right\} } & =R_{0}^{2 \prime}\left(\frac{1}{\mathrm{c} \overline{\mathrm{c}}}\right)\left(\frac{\overline{\mathrm{c}}}{\mathrm{c}}\right)=\frac{1}{\mathrm{cc}} R_{0}^{2 \prime}, \\
{[\rho \wedge \zeta]\left\{d \kappa_{0}^{\prime}\right\} } & =K_{0}^{2 \prime}\left(\frac{1}{\mathrm{c} \overline{\mathrm{c}}}\right)\left(\frac{\overline{\mathrm{c}}}{\mathrm{c}}\right)+K_{0}^{5 \prime}\left(i \frac{\mathrm{e}}{\mathrm{cc}}\right)\left(\frac{\overline{\mathrm{c}}}{\mathrm{c}}\right)-\left(\frac{\overline{\mathrm{c}}}{\mathrm{c}}\right)\left(-i \frac{\overline{\mathrm{e}}}{\overline{\mathrm{cc}}}\right) \\
& =\frac{1}{\mathrm{cc}} K_{0}^{2 \prime}+i \frac{\overline{\mathrm{c}}}{\mathrm{ccc}} K_{0}^{5 \prime}+i \frac{\overline{\mathrm{e}}}{\mathrm{c} \overline{\mathrm{c}}},
\end{aligned}
$$

hence

$$
\begin{aligned}
K^{2}= & -i \frac{\overline{\mathrm{ce}}}{\mathrm{cc}} R_{0}^{2 \prime}+\frac{1}{\mathrm{c}} K_{0}^{2 \prime}+i \frac{\overline{\mathrm{ce}}}{\mathrm{cc}} K_{0}^{5 \prime}+i \frac{\overline{\mathrm{e}}}{\overline{\mathrm{c}}} \\
= & i \frac{\overline{\mathrm{e}}}{\overline{\mathrm{c}}}+\frac{1}{\mathrm{c}}\left(-\frac{i}{3} \frac{\mathcal{K}\left(\overline{\mathcal{L}}_{1}\left(\overline{\mathcal{L}}_{1}(k)\right)\right)}{\overline{\mathcal{L}}_{1}(k)^{2}}+\frac{i}{3} \frac{\mathcal{K}\left(\overline{\mathcal{L}}_{1}(k)\right) \overline{\mathcal{L}}_{1}\left(\overline{\mathcal{L}}_{1}(k)\right)}{\overline{\mathcal{L}}_{1}(k)^{3}}\right. \\
& \left.-\frac{i}{3} \frac{\mathcal{L}_{1}\left(\mathcal{L}_{1}(\bar{k})\right)}{\mathcal{L}_{1}(\bar{k})}-\frac{i}{3} \frac{\overline{\mathcal{L}}_{1}\left(\mathcal{L}_{1}(k)\right)}{\overline{\mathcal{L}}_{1}(k)}-\frac{2}{3} \frac{\mathcal{T}(k)}{\overline{\mathcal{L}}_{1}(k)}\right) .
\end{aligned}
$$

Next, treat

$$
Z^{5}=[\kappa \wedge \zeta]\left\{\mathrm{d} d \rho_{0}+\mathrm{e} d \kappa_{0}^{\prime}+\frac{\mathrm{c}}{\overline{\mathrm{c}}} d \zeta_{0}^{\prime}\right\},
$$

using

$$
\begin{aligned}
& {[\kappa \wedge \zeta]\left\{d \rho_{0}\right\}=0,} \\
& {[\kappa \wedge \zeta]\left\{d \kappa_{0}^{\prime}\right\}=K_{0}^{5 \prime}\left(\frac{1}{\mathrm{c}}\right)\left(\frac{\overline{\mathrm{c}}}{\mathrm{c}}\right)=\frac{\overline{\mathrm{c}}}{\mathrm{cc}} K_{0}^{5 \prime},} \\
& {[\kappa \wedge \zeta]\left\{d \zeta_{0}^{\prime}\right\}=Z_{0}^{5 \prime}\left(\frac{1}{\mathrm{c}}\right)\left(\frac{\overline{\mathrm{c}}}{\mathrm{c}}\right)=\frac{\overline{\mathrm{c}}}{\mathrm{cc}} Z_{0}^{5 \prime},}
\end{aligned}
$$


so

Lastly treat

$$
Z^{5}=\frac{\overline{\mathrm{ce}}}{\mathrm{cc}}\left(-\frac{\mathcal{L}_{1}(k)}{\overline{\mathcal{L}}_{1}(k)}\right)+\frac{1}{\mathrm{c}}\left(\frac{\mathcal{L}_{1}\left(\overline{\mathcal{L}}_{1}(k)\right)}{\overline{\mathcal{L}}_{1}(k)}\right)
$$

using

$$
Z^{8}=[\zeta \wedge \bar{\kappa}]\left\{\mathrm{d} d \rho_{0}+\mathrm{e} d \kappa_{0}^{\prime}+\frac{\mathrm{c}}{\overline{\mathrm{c}}} d \zeta_{0}^{\prime}\right\},
$$

$$
\begin{aligned}
& {[\zeta \wedge \bar{\kappa}]\left\{d \rho_{0}\right\}=0} \\
& {[\zeta \wedge \bar{\kappa}]\left\{d \kappa_{0}^{\prime}\right\}=\frac{\overline{\mathrm{c}}}{\mathrm{c}} \frac{1}{\overline{\mathrm{c}}}=\frac{1}{\mathrm{c}}} \\
& {[\zeta \wedge \bar{\kappa}]\left\{d \zeta_{0}^{\prime}\right\}=Z_{0}^{8 \prime} \frac{\overline{\mathrm{c}}}{\overline{\mathrm{c}}} \frac{1}{\overline{\mathrm{c}}}+Z_{0}^{9 \prime} \frac{\overline{\mathrm{c}}}{\mathrm{c}}\left(-\frac{\mathrm{c} \overline{\mathrm{e}}}{\overline{\mathrm{cc}}}\right)=\frac{1}{\mathrm{c}} Z_{0}^{8 \prime}-\frac{\overline{\mathrm{e}}}{\overline{\mathrm{c}}} Z_{0}^{9 \prime},}
\end{aligned}
$$

which concludes

$$
\begin{aligned}
Z^{8} & =\frac{\mathrm{e}}{\mathrm{c}}+\frac{1}{\overline{\mathrm{c}}} Z_{0}^{8 \prime}-\frac{\mathrm{c} \overline{\mathrm{e}}}{\overline{\mathrm{cC}}} Z_{0}^{9 \prime} \\
& =\frac{\mathrm{e}}{\mathrm{c}}-\frac{1}{\overline{\mathrm{c}}} \frac{\overline{\mathcal{L}}_{1}\left(\overline{\mathcal{L}}_{1}(k)\right)}{\overline{\mathcal{L}}_{1}(k)}-\frac{\mathrm{c} \overline{\mathrm{e}}}{\overline{\mathrm{cC}}} \frac{\overline{\mathcal{L}}_{1}(k)}{\mathcal{L}_{1}(\bar{k})} .
\end{aligned}
$$

Thanks to these explicit expressions, we can compute the essential linear combination of torsion terms, emphasizing two important annihilations by pairs

$$
\begin{aligned}
-i K^{2}+Z^{5}-\bar{Z}^{8}= & \frac{\overline{\mathrm{e}}}{\mathrm{c}_{\circ}}+\frac{1}{\mathrm{c}}\left(-\frac{1}{3} \frac{\mathcal{K}\left(\overline{\mathcal{L}}_{1}\left(\overline{\mathcal{L}}_{1}(k)\right)\right)}{\overline{\mathcal{L}}_{1}(k)^{2}}+\frac{1}{3} \frac{\mathcal{K}\left(\overline{\mathcal{L}}_{1}(k)\right) \overline{\mathcal{L}}_{1}\left(\overline{\mathcal{L}}_{1}(k)\right)}{\overline{\mathcal{L}}_{1}(k)^{3}}\right. \\
& \left.-\frac{1}{3} \frac{\mathcal{L}_{1}\left(\mathcal{L}_{1}(\bar{k})\right)}{\mathcal{L}_{1}(\bar{k})}-\frac{1}{3} \frac{\overline{\mathcal{L}}_{1}\left(\mathcal{L}_{1}(k)\right)}{\overline{\mathcal{L}}_{1}(k)}+\frac{2 i}{3} \frac{\mathcal{T}(k)}{\overline{\mathcal{L}}_{1}(k)}\right) \\
& +\frac{1}{\mathrm{c}} \frac{\mathcal{L}_{1}\left(\overline{\mathcal{L}}_{1}(k)\right)}{\overline{\mathcal{L}}_{1}(k)}-\frac{\overline{\mathrm{ce}}}{\mathrm{cc}} \frac{\mathcal{L}_{1}(\bar{k})}{\overline{\mathcal{L}}_{1}(k)} \\
& -\frac{\overline{\mathrm{e}}}{\overline{\bar{c}}_{\circ}}+\frac{1}{\mathrm{c}} \frac{\mathcal{L}_{1}\left(\mathcal{L}_{1}(\bar{k})\right)}{\mathcal{L}_{1}(\bar{k})}+\frac{\overline{\mathrm{ce}} \frac{\mathcal{L}_{1}(\bar{k})}{\overline{\mathrm{cc}}\left(\overline{\mathcal{L}_{1}}\right)} .}{\overline{\mathcal{L}}_{1}(k)} .
\end{aligned}
$$

Also, in order to match exactly with Pocchiola's function $W$ introduced in $[25,18]$, we decompose the last term of the second line as

$$
\frac{2 i}{3} \frac{\mathcal{T}(k)}{\overline{\mathcal{L}}_{1}(k)}=-\frac{1}{3} \frac{\mathcal{L}_{1}\left(\overline{\mathcal{L}}_{1}(k)\right)}{\overline{\mathcal{L}}_{1}(k)}+\frac{1}{3} \frac{\overline{\mathcal{L}}_{1}\left(\mathcal{L}_{1}(k)\right)}{\overline{\mathcal{L}}_{1}(k)}+\frac{i}{3} \frac{\mathcal{T}(k)}{\overline{\mathcal{L}}_{1}(k)},
$$

so that a third pair of terms disappears, and after reorganization - no pen needed - , the result is

$$
\begin{aligned}
-i K^{2}+Z^{5}-\bar{Z}^{8}= & \frac{1}{\mathrm{c}}\left(-\frac{1}{3} \frac{\mathcal{K}\left(\overline{\mathcal{L}}_{1}\left(\overline{\mathcal{L}}_{1}(k)\right)\right)}{\overline{\mathcal{L}}_{1}(k)^{2}}+\frac{1}{3} \frac{\mathcal{K}\left(\overline{\mathcal{L}}_{1}(k)\right) \overline{\mathcal{L}}_{1}\left(\overline{\mathcal{L}}_{1}(k)\right)}{\overline{\mathcal{L}}_{1}(k)^{3}}\right. \\
& \left.+\frac{2}{3} \frac{\mathcal{L}_{1}\left(\mathcal{L}_{1}(\bar{k})\right)}{\mathcal{L}_{1}(\bar{k})}+\frac{2}{3} \frac{\mathcal{L}_{1}\left(\overline{\mathcal{L}}_{1}(k)\right)}{\overline{\mathcal{L}}_{1}(k)}+\frac{i}{3} \frac{\mathcal{T}(k)}{\overline{\mathcal{L}}_{1}(k)}\right) \\
=: & \frac{1}{\mathrm{c}} W_{0},
\end{aligned}
$$

and this defines a new horizontal function $W_{0}$, equal to Pocchiola's function $W$. 
For now, we will not use the potential normalization $c=W_{0}$ on the open subset of $M^{5} \subset \mathbb{C}^{3}$ on which

$$
0 \neq W_{0}\left(z_{1}, z_{2}, \bar{z}_{1}, \bar{z}_{2}, v\right)
$$

if nonempty - a hypothesis must be set up - , but we will deal with this discussion later. In fact, before proceeding, we state a technical differential relation useful later, whose proof can be skipped in a first reading.

Lemma 8.8. One has

$$
\overline{\mathcal{K}}\left(H_{0}\right)=-2 \overline{\mathcal{L}}_{1}(\bar{k}) H_{0} .
$$

Proof. Apply the derivation $\overline{\mathcal{K}}$ to $H_{0}$

$$
\begin{aligned}
\overline{\mathcal{K}}\left(H_{0}\right)= & -\frac{1}{6} \frac{\overline{\mathcal{K}}\left(\overline{\mathcal{L}}_{1}\left(\overline{\mathcal{L}}_{1}\left(\overline{\mathcal{L}}_{1}(k)\right)\right)\right)}{\overline{\mathcal{L}}_{1}(k)}+\frac{1}{6} \frac{\overline{\mathcal{K}}\left(\overline{\mathcal{L}}_{1}(k)\right) \overline{\mathcal{L}}_{1}\left(\overline{\mathcal{L}}_{1}\left(\overline{\mathcal{L}}_{1}(k)\right)\right)}{\overline{\mathcal{L}}_{1}(k)^{2}} \\
& +\frac{4}{9} \frac{\overline{\mathcal{K}}\left(\overline{\mathcal{L}}_{1}\left(\overline{\mathcal{L}}_{1}(k)\right)\right) \overline{\mathcal{L}}_{1}\left(\overline{\mathcal{L}}_{1}(k)\right)}{\overline{\mathcal{L}}_{1}(k)^{2}}-\frac{4}{9} \frac{\overline{\mathcal{K}}\left(\overline{\mathcal{L}}_{1}(k)\right) \overline{\mathcal{L}}_{1}\left(\overline{\mathcal{L}}_{1}(k)\right)^{2}}{\overline{\mathcal{L}}_{1}(k)^{3}} \\
& +\frac{1}{18} \frac{\overline{\mathcal{K}}\left(\overline{\mathcal{L}}_{1}\left(\overline{\mathcal{L}}_{1}(k)\right)\right)}{\overline{\mathcal{L}}_{1}(k)} \bar{P}+\frac{1}{18} \frac{\overline{\mathcal{L}}_{1}\left(\overline{\mathcal{L}}_{1}(k)\right) \overline{\mathcal{K}}(\bar{P})}{\overline{\mathcal{L}}_{1}(k)} \\
& -\frac{1}{18} \frac{\overline{\mathcal{K}}\left(\overline{\mathcal{L}}_{1}(k)\right) \overline{\mathcal{L}}_{1}\left(\overline{\mathcal{L}}_{1}(k)\right) \bar{P}}{\overline{\mathcal{L}}_{1}(k)^{2}}+\frac{1}{6} \overline{\mathcal{K}}\left(\overline{\mathcal{L}}_{1}(\bar{P})\right)-\frac{2}{9} \bar{P} \overline{\mathcal{K}}(\bar{P})
\end{aligned}
$$

perform replacements using Lemmas 2.9 and 2.5

$$
\begin{aligned}
\overline{\mathcal{K}}\left(H_{0}\right)= & \frac{1}{2} \frac{\overline{\mathcal{L}}_{1}(\bar{k}) \overline{\mathcal{L}}_{1}\left(\overline{\mathcal{L}}_{1}\left(\overline{\mathcal{L}}_{1}(k)\right)\right)}{\overline{\mathcal{L}}_{1}(k)}+\frac{1}{2} \frac{\overline{\mathcal{L}}_{1}\left(\overline{\mathcal{L}}_{1}(\bar{k})\right) \overline{\mathcal{L}}_{1}\left(\overline{\mathcal{L}}_{1}(k)\right)}{\overline{\mathcal{L}}_{1}(k)}+\frac{1}{6} \overline{\mathcal{L}}_{1}\left(\overline{\mathcal{L}}_{1}\left(\overline{\mathcal{L}}_{1}(\bar{k})\right)\right)-\frac{1}{6} \frac{\overline{\mathcal{L}}_{1}(\bar{k}) \overline{\mathcal{L}}_{1}\left(\overline{\mathcal{L}}_{1}\left(\overline{\mathcal{L}}_{1}(k)\right)\right)}{\overline{\mathcal{L}}_{1}(k)} \\
& -\frac{8}{9} \frac{\overline{\mathcal{L}}_{1}\left(\overline{\mathcal{L}}_{1}(k)\right)^{2} \overline{\mathcal{L}}_{1}(\bar{k})}{\left.\overline{\mathcal{L}}_{1}(k)\right)^{2}}-\frac{4}{9} \frac{\overline{\mathcal{L}}_{1}\left(\overline{\mathcal{L}}_{1}(\bar{k})\right) \overline{\mathcal{L}}_{1}\left(\overline{\mathcal{L}}_{1}(k)\right)}{\overline{\mathcal{L}}_{1}(k)}+\frac{4}{9} \frac{\overline{\mathcal{L}}_{1}(\bar{k}) \overline{\mathcal{L}}_{1}\left(\overline{\mathcal{L}}_{1}(k)\right)^{2}}{\overline{\mathcal{L}}_{1}(k)^{2}} \\
& -\frac{1}{9} \frac{\overline{\mathcal{L}}_{1}\left(\overline{\mathcal{L}}_{1}(k)\right) \overline{\mathcal{L}}_{1}(\bar{k}) \bar{P}}{\overline{\mathcal{L}}_{1}(k)}-\frac{1}{18} \overline{\mathcal{L}}_{1}\left(\overline{\mathcal{L}}_{1}(\bar{k})\right) \bar{P}-\frac{1}{18} \frac{\overline{\mathcal{L}}_{1}\left(\overline{\mathcal{L}}_{1}(k)\right) \overline{\mathcal{L}}_{1}(\bar{k}) \bar{P}}{\overline{\mathcal{L}}_{1}(k)}-\frac{1}{\frac{18}{18}} \div \frac{\overline{\mathcal{L}}_{1}\left(\overline{\mathcal{L}}_{1}(k)\right) \overline{\mathcal{L}}_{1}\left(\overline{\mathcal{L}}_{1}(\bar{k})\right)}{\overline{\mathcal{L}}_{1}(k)} \\
& +\frac{1}{18} \frac{\overline{\mathcal{L}}_{1}(\bar{k}) \overline{\mathcal{L}}_{1}\left(\overline{\mathcal{L}}_{1}(k)\right) \bar{P}}{\overline{\mathcal{L}}_{1}(k)}+\frac{1}{6} \overline{\mathcal{K}}\left(\overline{\mathcal{L}}_{1}(\bar{P})\right)+\frac{2}{9} \bar{P} \bar{P} \overline{\mathcal{L}}_{1}(\bar{k})+\frac{2}{9} \bar{P}_{\overline{\mathcal{L}}_{1}\left(\overline{\mathcal{L}}_{1}(\bar{k})\right)}^{\circ}
\end{aligned}
$$

and observe some (underlined) cancellations to get an expression in which the last three terms must yet be transformed

$$
\begin{aligned}
\overline{\mathcal{K}}\left(H_{0}\right)= & \frac{1}{3} \frac{\overline{\mathcal{L}}_{1}(\bar{k}) \overline{\mathcal{L}}_{1}\left(\overline{\mathcal{L}}_{1}\left(\overline{\mathcal{L}}_{1}(k)\right)\right)}{\overline{\mathcal{L}}_{1}(k)}-\frac{4}{9} \frac{\overline{\mathcal{L}}_{1}(\bar{k}) \overline{\mathcal{L}}_{1}\left(\overline{\mathcal{L}}_{1}(k)\right)^{2}}{\overline{\mathcal{L}}_{1}(k)^{2}}-\frac{1}{9} \frac{\overline{\mathcal{L}}_{1}(\bar{k}) \overline{\mathcal{L}}_{1}\left(\overline{\mathcal{L}}_{1}(k)\right) \bar{P}}{\overline{\mathcal{L}}_{1}(k)} \\
& +\frac{2}{9} \bar{P} \bar{P} \overline{\mathcal{L}}_{1}(\bar{k})+\frac{1}{6} \overline{\mathcal{L}}_{1}\left(\overline{\mathcal{L}}_{1}\left(\overline{\mathcal{L}}_{1}(\bar{k})\right)\right)+\frac{1}{6} \overline{\mathcal{L}}_{1}\left(\overline{\mathcal{L}}_{1}(k)\right) \bar{P}+\frac{1}{6} \overline{\mathcal{K}}\left(\overline{\mathcal{L}}_{1}(\bar{P})\right) .
\end{aligned}
$$

Lemma 8.9. One has

$$
\overline{\mathcal{L}}_{1}\left(\overline{\mathcal{L}}_{1}\left(\overline{\mathcal{L}}_{1}(\bar{k})\right)\right)+\overline{\mathcal{L}}_{1}\left(\overline{\mathcal{L}}_{1}(k)\right) \bar{P}+\overline{\mathcal{K}}\left(\overline{\mathcal{L}}_{1}(\bar{P})\right)=-2 \overline{\mathcal{L}}_{1}(\bar{k}) \overline{\mathcal{L}}_{1}(\bar{P}) .
$$

Proof. Apply the vector field $\overline{\mathcal{L}}_{1}$ to Lemma 2.5

$$
\overline{\mathcal{L}}_{1}(\overline{\mathcal{K}}(\bar{P}))=-\overline{\mathcal{L}}_{1}(\bar{P}) \overline{\mathcal{L}}_{1}(\bar{k})-\bar{P} \overline{\mathcal{L}}_{1}\left(\overline{\mathcal{L}}_{1}(\bar{k})\right)-\overline{\mathcal{L}}_{1}\left(\overline{\mathcal{L}}_{1}\left(\overline{\mathcal{L}}_{1}(\bar{k})\right)\right) .
$$

On the other hand, apply the Lie bracket $\left[\overline{\mathcal{L}}_{1}, \overline{\mathcal{K}}\right](\bullet)$ to the function $\bar{P}$, using the concerned known commutation relation shown in Section 2

$$
\overline{\mathcal{L}}_{1}(\overline{\mathcal{K}}(\bar{P}))-\overline{\mathcal{K}}\left(\overline{\mathcal{L}}_{1}(\bar{P})\right)=\left[\overline{\mathcal{L}}_{1}, \overline{\mathcal{K}}\right](\bar{P})=\overline{\mathcal{L}}_{1}(\bar{k}) \overline{\mathcal{L}}_{1}(\bar{P})
$$


and replace the first term $\overline{\mathcal{L}}_{1}(\overline{\mathcal{K}}(\bar{P}))$ by its value above to get the result.

Consequently, after this transformation, we see that $\overline{\mathcal{K}}\left(H_{0}\right)$ is a multiple of $\overline{\mathcal{L}}_{1}(\bar{k})$ in which we recognize $-2 H_{0}$ as stated

$$
\overline{\mathcal{K}}\left(H_{0}\right)=\overline{\mathcal{L}}_{1}(\bar{k})\left(\frac{1}{3} \frac{\overline{\mathcal{L}}_{1}\left(\overline{\mathcal{L}}_{1}\left(\overline{\mathcal{L}}_{1}(k)\right)\right)}{\overline{\mathcal{L}}_{1}(k)}-\frac{4}{9} \frac{\overline{\mathcal{L}}_{1}\left(\overline{\mathcal{L}}_{1}(k)\right)^{2}}{\overline{\mathcal{L}}_{1}(k)^{2}}-\frac{1}{9} \frac{\overline{\mathcal{L}}_{1}\left(\overline{\mathcal{L}}_{1}(k)\right) \bar{P}}{\overline{\mathcal{L}}_{1}(k)}-\frac{1}{3} \overline{\mathcal{L}_{1}}(\bar{P})+\frac{2}{9} \bar{P}^{2}\right) .
$$

As we already observed, the essential (invariant) torsion $i K^{3}-Z^{6}$ can be set 0 to normalize the group parameter $d$ as

whence inserting in (8.1)

$$
\mathrm{d}:=-\frac{i}{2} \frac{\bar{c} e \mathrm{e}}{\mathrm{c}}+i \frac{\mathrm{c}}{\overline{\mathrm{c}}} H_{0}
$$

$$
\left(\begin{array}{c}
\rho \\
\kappa \\
\zeta
\end{array}\right):=\left(\begin{array}{ccc}
c \bar{c} & 0 & 0 \\
-i \overline{\mathrm{c} e} & \mathrm{c} & 0 \\
-\frac{i}{2} \frac{\overline{\mathrm{cee}}}{\mathrm{c}}+i \frac{\bar{c}}{\bar{c}} H_{0} & \mathrm{e} & \overline{\bar{c}}
\end{array}\right)\left(\begin{array}{c}
\rho_{0} \\
\kappa_{0}^{\prime} \\
\zeta_{0}^{\prime}
\end{array}\right) .
$$

Thus, we are naturally led to change the initial coframe on $M$

$$
\left\{\rho_{0}, \kappa_{0}^{\prime}, \zeta_{0}^{\prime}, \bar{\kappa}_{0}^{\prime}, \bar{\zeta}_{0}^{\prime}\right\} \rightsquigarrow\left\{\rho_{0}, \kappa_{0}^{\prime}, \zeta_{0}^{\prime \prime}, \bar{\kappa}_{0}^{\prime}, \bar{\zeta}_{0}^{\prime \prime}\right\},
$$

by introducing the new 1 -form

$$
\zeta_{0}^{\prime \prime}:=\zeta_{0}^{\prime}+i H_{0} \rho_{0},
$$

so that a new, reduced by two real dimensions, $G$-structure, appears

$$
\left(\begin{array}{c}
\rho \\
\kappa \\
\zeta
\end{array}\right):=\left(\begin{array}{ccc}
\mathrm{c} \overline{\mathrm{c}} & 0 & 0 \\
-i \overline{\mathrm{ce}} & \mathrm{c} & 0 \\
-\frac{i}{2} \frac{\overline{\mathrm{cee}}}{\mathrm{c}} & \mathrm{e} & \overline{\bar{c}}
\end{array}\right)\left(\begin{array}{c}
\rho_{0} \\
\kappa_{0}^{\prime} \\
\zeta_{0}^{\prime \prime}
\end{array}\right),
$$

which is justified by the computation/reorganization

$$
\begin{aligned}
\zeta & =\left(-\frac{i}{2} \frac{\overline{\mathrm{cee}}}{\mathrm{c}}+i \frac{\mathrm{c}}{\overline{\mathrm{c}}} H_{0}\right) \rho_{0}+\mathrm{e} \kappa_{0}^{\prime}+\frac{\mathrm{c}}{\overline{\mathrm{c}}} \zeta_{0}^{\prime} \\
& =-\frac{i}{2} \frac{\overline{\mathrm{c} e e}}{\mathrm{c}} \rho_{0}+\mathrm{e} \kappa_{0}^{\prime}+\frac{\mathrm{c}}{\overline{\mathrm{c}}}(\underbrace{\zeta_{0}^{\prime}+i H_{0} \rho_{0}}_{=: \zeta_{0}^{\prime \prime}}) .
\end{aligned}
$$

Back to previous expressions, this last coframe writes out as

$$
\begin{aligned}
\rho_{0} & :=\frac{1}{\ell}\left(d v-A^{1} d z_{1}-A^{2} d z_{2}-\bar{A}^{1} d \bar{z}_{1}-\bar{A}^{2} d \bar{z}_{2}\right), \\
\kappa_{0}^{\prime} & :=d z_{1}-k d z_{2}+\frac{i}{3} B_{0} \rho_{0}, \\
\zeta_{0}^{\prime \prime} & :=\overline{\mathcal{L}}_{1}(k) d z_{2}+i H_{0} \rho_{0} .
\end{aligned}
$$

9. DarbouX-Cartan Structure of the Coframe $\left\{\rho_{0}, \kappa_{0}^{\prime}, \zeta_{0}^{\prime \prime}, \bar{\kappa}_{0}^{\prime}, \bar{\zeta}_{0}^{\prime \prime}\right\}$

The present change of initial coframe expresses as

$$
\zeta_{0}^{\prime \prime}:=\zeta_{0}^{\prime}+i H_{0} \rho_{0} \quad \Longleftrightarrow \quad \zeta_{0}^{\prime}=\zeta_{0}^{\prime \prime}-i H_{0} .
$$

The exterior differentiation of $\zeta_{0}^{\prime \prime}$ comprises 3 terms that we shall compute soon

$$
d \zeta_{0}^{\prime \prime}=d \zeta_{0}^{\prime}+i d \boldsymbol{H}_{0} \wedge \rho_{0}+i \boldsymbol{H}_{0} d \rho_{0} .
$$


Back to the previous structure equations written in the abbreviated form (7.1), we may start by replacing $\zeta_{0}^{\prime}$ in $d \rho_{0}$, while observing that

$$
\rho_{0} \wedge \zeta_{0}^{\prime}=\rho_{0} \wedge \zeta_{0}^{\prime \prime} \quad \text { and } \quad \rho_{0} \wedge \bar{\zeta}_{0}^{\prime}=\rho_{0} \wedge \bar{\zeta}_{0}^{\prime \prime},
$$

we come to unchanged coefficients for

$$
d \rho_{0}=R_{0}^{1 \prime} \rho_{0} \wedge \kappa_{0}^{\prime}+R_{0}^{2 \prime} \rho_{0} \wedge \zeta_{0}^{\prime \prime}+\bar{R}_{0}^{1 \prime} \rho_{0} \wedge \bar{\kappa}_{0}^{\prime}+\bar{R}_{0}^{2 \prime} \rho_{0} \wedge \bar{\zeta}_{0}^{\prime \prime}+i \kappa_{0}^{\prime} \wedge \bar{\kappa}_{0}^{\prime},
$$

hence without computation, the third term is

$i H_{0} d \rho_{0}=i H_{0} R_{0}^{1 \prime} \rho_{0} \wedge \kappa_{0}^{\prime}+i H_{0} R_{0}^{2 \prime} \rho_{0} \wedge \zeta_{0}^{\prime}+i H_{0} \bar{R}_{0}^{1 \prime} \rho_{0} \wedge \bar{\kappa}_{0}^{\prime}+i H_{0} \bar{R}_{0}^{2 \prime} \rho_{0} \wedge \bar{\zeta}_{0}^{\prime}-H_{0} \kappa_{0} \wedge \bar{\kappa}_{0}^{\prime}$.

Next, we do the same replacement of $\zeta_{0}^{\prime}$ in

$$
\begin{aligned}
d \kappa_{0}^{\prime}= & K_{0}^{1 \prime} \rho_{0} \wedge \kappa_{0}^{\prime}+K_{0}^{2 \prime} \rho_{0} \wedge\left(\zeta_{0}^{\prime \prime}-i H_{0} \rho_{0}\right)+K_{0}^{3 \prime} \rho_{0} \wedge \bar{\kappa}_{0}^{\prime} \\
& +K_{0}^{5 \prime} \kappa_{0}^{\prime} \wedge\left(\zeta_{0}^{\prime \prime}-i H_{0} \rho_{0}\right)+K_{0}^{6 \prime} \kappa_{0}^{\prime} \wedge \bar{\kappa}_{0}^{\prime}+\left(\zeta_{0}^{\prime \prime}-i H_{0} \rho_{0}\right) \wedge \bar{\kappa}_{0}^{\prime},
\end{aligned}
$$

hence

$$
\begin{aligned}
d \kappa_{0}^{\prime}= & (\underbrace{K_{0}^{1 \prime}+i K_{0}^{5 \prime} H_{0}}_{=: K_{0}^{1 \prime \prime}}) \rho_{0} \wedge \kappa_{0}^{\prime}+K_{0}^{2 \prime} \rho_{0} \wedge \zeta_{0}^{\prime \prime}+(\underbrace{K_{0}^{3 \prime}-i H_{0}}_{=: K_{0}^{3 \prime}}) \rho_{0} \wedge \bar{\kappa}_{0}^{\prime} \\
& +K_{0}^{5 \prime} \kappa_{0}^{\prime} \wedge \zeta_{0}^{\prime \prime}+K_{0}^{6 \prime} \kappa_{0}^{\prime} \wedge \bar{\kappa}_{0}^{\prime}+\zeta_{0}^{\prime \prime} \wedge \bar{\kappa}_{0}^{\prime} .
\end{aligned}
$$

Similarly, do the same for

$$
\begin{aligned}
d \zeta_{0}^{\prime}= & Z_{0}^{2 \prime} \rho_{0} \wedge\left(\zeta_{0}^{\prime \prime}-i H_{0} \rho_{0}\right)+Z_{0}^{5 \prime} \kappa_{0}^{\prime} \wedge\left(\bar{\zeta}_{0}^{\prime \prime}+i H_{0} \rho_{0}\right) \\
& +Z_{0}^{8 \prime}\left(\zeta_{0}^{\prime \prime}-i H_{0} \rho_{0}\right) \wedge \bar{\kappa}_{0}^{\prime}+Z_{0}^{9 \prime}\left(\zeta_{0}^{\prime \prime}-i H_{0} \rho_{0}\right) \wedge\left(\bar{\zeta}_{0}^{\prime \prime}+i \bar{H}_{0} \rho_{0}\right),
\end{aligned}
$$

hence

$$
\begin{aligned}
d \zeta_{0}^{\prime}= & i \boldsymbol{Z}_{0}^{5 \prime} H_{0} \rho_{0} \wedge \kappa_{0}^{\prime}+\left(\boldsymbol{Z}_{0}^{2 \prime}-i \boldsymbol{Z}_{0}^{9 \prime} \overline{\boldsymbol{H}}_{0}\right) \rho_{0} \wedge \zeta_{0}^{\prime \prime}-i \boldsymbol{Z}_{0}^{8 \prime} \boldsymbol{H}_{0} \rho_{0} \wedge \bar{\kappa}_{0}^{\prime} \\
& -i \boldsymbol{Z}_{0}^{9 \prime} \boldsymbol{H}_{0} \rho_{0} \wedge \bar{\zeta}_{0}^{\prime \prime}+\boldsymbol{Z}_{0}^{5 \prime} \kappa_{0}^{\prime} \wedge \zeta_{0}^{\prime \prime}+\boldsymbol{Z}_{0}^{8 \prime} \zeta_{0}^{\prime \prime} \wedge \bar{\kappa}_{0}^{\prime}+\boldsymbol{Z}_{0}^{9 \prime} \zeta_{0}^{\prime \prime} \wedge \bar{\zeta}_{0}^{\prime \prime} .
\end{aligned}
$$

Next, we have to compute the second term in $d \zeta_{0}^{\prime \prime}$, and using

$$
d H_{0}=\mathcal{T}\left(H_{0}\right) \rho_{0}+\mathcal{L}_{1}\left(H_{0}\right) \kappa_{0}+\mathcal{K}\left(H_{0}\right) \zeta_{0}+\overline{\mathcal{L}}_{1}\left(H_{0}\right) \bar{\kappa}_{0}+\overline{\mathcal{K}}\left(H_{0}\right) \bar{\zeta}_{0},
$$

it comes

$$
\begin{aligned}
d H_{0} \wedge \rho_{0}= & 0-\mathcal{L}_{1}\left(H_{0}\right) \rho_{0} \wedge \kappa_{0}-\mathcal{K}\left(H_{0}\right) \rho_{0} \wedge \zeta_{0}-\overline{\mathcal{L}}_{1}\left(H_{0}\right) \rho_{0} \wedge \bar{\kappa}_{0}-\overline{\mathcal{K}}\left(H_{0}\right) \rho_{0} \wedge \bar{\zeta}_{0} \\
= & -\mathcal{L}_{1}\left(H_{0}\right) \rho_{0} \wedge\left(\kappa_{0}^{\prime}-\frac{i}{3} B_{0} \rho_{0}\right)-\mathcal{K}\left(H_{0}\right) \rho_{0} \wedge \frac{\zeta_{0}^{\prime}}{\overline{\mathcal{L}}_{1}(k)}-\overline{\mathcal{L}}_{1}\left(H_{0}\right) \rho_{0} \wedge\left(\bar{\kappa}_{0}^{\prime}+\frac{i}{3} \bar{B}_{0} \rho_{0}\right) \\
& -\overline{\mathcal{K}}\left(H_{0}\right) \rho_{0} \wedge \frac{\bar{\zeta}_{0}^{\prime}}{\mathcal{L}_{1}(\bar{k})} \\
= & -\mathcal{L}_{1}\left(H_{0}\right) \rho_{0} \wedge \kappa_{0}^{\prime}-\frac{\mathcal{K}\left(H_{0}\right)}{\overline{\mathcal{L}}_{1}(k)} \rho_{0} \wedge \zeta_{0}^{\prime}-\overline{\mathcal{L}}_{1}\left(H_{0}\right) \rho_{0} \wedge \bar{\kappa}_{0}^{\prime}-\frac{\overline{\mathcal{K}}\left(H_{0}\right)}{\mathcal{L}_{1}(\bar{k})} \rho_{0} \wedge \bar{\zeta}_{0}^{\prime},
\end{aligned}
$$

hence multiplying by $i$, we get the expression of the second term

$$
i d H_{0} \wedge \rho_{0}=-i \mathcal{L}_{1}\left(H_{0}\right) \rho_{0} \wedge \kappa_{0}^{\prime}-i \frac{\mathcal{K}\left(H_{0}\right)}{\overline{\mathcal{L}}_{1}(k)} \rho_{0} \wedge \zeta_{0}^{\prime \prime}-i \overline{\mathcal{L}}_{1}\left(H_{0}\right) \rho_{0} \wedge \bar{\kappa}_{0}^{\prime}-i \frac{\overline{\mathcal{K}}\left(\boldsymbol{H}_{0}\right)}{\mathcal{L}_{1}(\bar{k})} \rho_{0} \wedge \bar{\zeta}_{0}^{\prime \prime} .
$$


Summing and collecting the three computed terms yields

$$
\begin{aligned}
d \zeta_{0}^{\prime \prime}= & \rho_{0} \wedge \kappa_{0}^{\prime}[\underbrace{i \boldsymbol{Z}_{0}^{5 \prime} H_{0}-i \mathcal{L}_{1}\left(H_{0}\right)+i H_{0} R_{0}^{1 \prime}}_{=: Z_{0}^{1 \prime \prime}}]+\rho_{0} \wedge \zeta_{0}^{\prime \prime}[\underbrace{Z_{0}^{2 \prime}-i \boldsymbol{Z}_{0}^{9 \prime} \bar{H}_{0}-i \frac{\mathcal{K}\left(H_{0}\right)}{\overline{\mathcal{L}}_{1}(k)}+i H_{0} R_{0}^{2 \prime}}_{=: Z_{0}^{2 \prime \prime}}] \\
& +\rho_{0} \wedge \bar{\kappa}_{0}^{\prime}[\underbrace{-i \boldsymbol{Z}_{0}^{8 \prime} H_{0}-i \overline{\mathcal{L}}_{1}\left(H_{0}\right)+i H_{0} \overline{\boldsymbol{R}}_{0}^{1 \prime}}_{=: Z_{0}^{3 \prime \prime}}]+\rho_{0} \wedge \bar{\zeta}_{0}^{\prime \prime}[\underbrace{\left[-i \boldsymbol{Z}_{0}^{9 \prime} H_{0}-i \frac{\overline{\mathcal{K}}\left(H_{0}\right)}{\mathcal{L}_{1}(\bar{k})}+i H_{0} \bar{R}_{0}^{2 \prime}\right.}_{=: Z_{0}^{\prime \prime}}] \\
& +Z_{0}^{5 \prime} \kappa_{0}^{\prime} \wedge \zeta_{0}^{\prime \prime}+\kappa_{0}^{\prime} \wedge \bar{\kappa}_{0}^{\prime}[\underbrace{-H_{0}}_{0}]+\boldsymbol{Z}_{0}^{8 \prime} \zeta_{0}^{\prime \prime} \wedge \bar{\kappa}_{0}^{\prime}+\boldsymbol{Z}_{0}^{9 \prime} \zeta_{0}^{\prime \prime} \wedge \bar{\zeta}_{0}^{\prime \prime} .
\end{aligned}
$$

Lemma 9.1. One has the identical vanishing of the coefficient of $\rho_{0} \wedge \bar{\zeta}_{0}^{\prime \prime}$ in $d \zeta_{0}^{\prime \prime}$

$$
\begin{aligned}
Z_{0}^{4 \prime \prime} & :=-i Z_{0}^{9 \prime} H_{0}-i \frac{\overline{\mathcal{K}}\left(H_{0}\right)}{\mathcal{L}_{1}(\bar{k})}+i H_{0} \bar{R}_{0}^{2 \prime} \\
& \equiv 0 .
\end{aligned}
$$

Proof. This is equivalent to

$$
\overline{\mathcal{K}}\left(H_{0}\right) \stackrel{?}{=} \mathcal{L}_{1}(\bar{k}) H_{0}\left(-Z_{0}^{9 \prime}+\bar{R}_{0}^{2 \prime}\right)
$$

and after a replacement using Proposition 7.2, to

$$
\overline{\mathcal{K}}\left(H_{0}\right) \stackrel{?}{\equiv} \mathcal{L}_{1}(\bar{k}) H_{0}\left(-\frac{\overline{\mathcal{L}}_{1}(\bar{k})}{\mathcal{L}_{1}(\bar{k})}-\frac{\overline{\mathcal{L}}_{1}(\bar{k})}{\mathcal{L}_{1}(\bar{k})}\right),
$$

an identity which was already seen by Lemma 8.8.

In summary

$$
\begin{aligned}
d \rho_{0}= & R_{0}^{1 \prime} \rho_{0} \wedge \kappa_{0}^{\prime}+R_{0}^{2 \prime} \rho_{0} \wedge \zeta_{0}^{\prime \prime}+\bar{R}_{0}^{1 \prime} \rho_{0} \wedge \bar{\kappa}_{0}^{\prime}+\bar{R}_{0}^{2 \prime} \rho_{0} \wedge \bar{\zeta}_{0}^{\prime \prime}+i \kappa_{0}^{\prime} \wedge \bar{\kappa}_{0}^{\prime}, \\
d \kappa_{0}^{\prime}= & K_{0}^{1 \prime \prime} \rho_{0} \wedge \kappa_{0}^{\prime}+K_{0}^{2 \prime} \rho_{0} \wedge \zeta_{0}^{\prime \prime}+K_{0}^{3 \prime \prime} \rho_{0} \wedge \bar{\kappa}_{0}^{\prime} \\
& +K_{0}^{5 \prime} \kappa_{0}^{\prime} \wedge \zeta_{0}^{\prime \prime}+K_{0}^{6 \prime} \kappa_{0}^{\prime} \wedge \bar{\kappa}_{0}^{\prime}+\zeta_{0}^{\prime \prime} \wedge \bar{\kappa}_{0}^{\prime}, \\
d \zeta_{0}^{\prime \prime}= & Z_{0}^{1 \prime \prime} \rho_{0} \wedge \kappa_{0}^{\prime}+Z_{0}^{2 \prime \prime} \rho_{0} \wedge \zeta_{0}^{\prime \prime}+Z_{0}^{3 \prime \prime} \rho_{0} \wedge \bar{\kappa}_{0}^{\prime} \\
& +Z_{0}^{5 \prime} \kappa_{0}^{\prime} \wedge \zeta_{0}^{\prime \prime}+Z_{0}^{6 \prime \prime} \kappa_{0}^{\prime} \wedge \bar{\kappa}_{0}^{\prime}+Z_{0}^{8 \prime} \zeta_{0}^{\prime \prime} \wedge \bar{\kappa}_{0}^{\prime}+Z_{0}^{9 \prime} \zeta_{0}^{\prime \prime} \wedge \bar{\zeta}_{0}^{\prime \prime} .
\end{aligned}
$$

Notice that new coefficients $Z_{0}^{2 \prime \prime}, Z_{0}^{3 \prime \prime}, Z_{0}^{4 \prime \prime}$ appear in $d \zeta_{0}^{\prime \prime}$, which were absent in $d \zeta_{0}^{\prime}$, as they are coming from the second term $i d H_{0} \wedge \rho_{0}$.

\section{ABSORPTION AND APPARITION OF TWO 1 -FORMS $\pi^{1}, \pi^{2}$}

With the 4-dimensional group parametrized by $(c, \bar{c}, e, \bar{e})$, the lifted coframe writes:

$$
\left(\begin{array}{c}
\rho \\
\kappa \\
\zeta
\end{array}\right):=\left(\begin{array}{ccc}
\mathrm{c} \overline{\mathrm{c}} & 0 & 0 \\
-i \overline{\mathrm{c} e} & \mathrm{c} & 0 \\
-\frac{i}{2} \frac{\mathrm{cee}}{\mathrm{c}} & \mathrm{e} & \overline{\bar{c}}
\end{array}\right)\left(\begin{array}{c}
\rho_{0} \\
\kappa_{0}^{\prime} \\
\zeta_{0}^{\prime \prime}
\end{array}\right) \quad \Longleftrightarrow \quad\left\{\begin{array}{l}
\rho:=\mathrm{c} \overline{\mathrm{c}} \rho_{0}, \\
\kappa:=-i \overline{\mathrm{c} e} \rho_{0}+\mathrm{c} \kappa_{0}^{\prime}, \\
\zeta:=-\frac{i}{2} \frac{\overline{\mathrm{cee}}}{\mathrm{c}} \rho_{0}+\mathrm{e} \kappa_{0}^{\prime}+\frac{\mathrm{c}}{\overline{\mathrm{c}}} \zeta_{0}^{\prime \prime},
\end{array}\right.
$$


with inverse formulas

$$
\begin{aligned}
\rho_{0} & =\frac{1}{\mathrm{c} \overline{\mathrm{c}}} \rho, \\
\kappa_{0}^{\prime} & =i \frac{\mathrm{e}}{\mathrm{cc}} \rho+\frac{1}{\mathrm{c}} \kappa, \\
\zeta_{0}^{\prime \prime} & =-\frac{i}{2} \frac{\overline{\mathrm{cee}}}{\mathrm{ccc}} \rho-\frac{\overline{\mathrm{ce}}}{\mathrm{cc}} \kappa+\frac{\overline{\mathrm{c}}}{\mathrm{c}} \zeta .
\end{aligned}
$$

The Maurer-Cartan matrix becomes

$$
\begin{aligned}
& d g \cdot g^{-1}=\left(\begin{array}{ccc}
\bar{c} d c+c d \bar{c} & 0 & 0 \\
-i \text { e } d \bar{c}-i \bar{c} d e & d c & 0 \\
-\frac{i}{2} \frac{\text { ee } d \bar{c}}{c}-i \frac{\bar{c} e d e}{c}+\frac{i}{2} \frac{\overline{c e e} d c}{c c} & d e & \frac{d c}{\bar{c}}-\frac{c d \bar{c}}{\overline{c c}}
\end{array}\right)\left(\begin{array}{ccc}
\frac{1}{c \bar{c}} & 0 & 0 \\
i \frac{e}{c c} & \frac{1}{c} & 0 \\
-\frac{i}{2} \frac{\bar{c}}{c c e} & -\frac{\overline{c e}}{c c} & \frac{\bar{c}}{c}
\end{array}\right) \\
& =:\left(\begin{array}{ccc}
\alpha+\bar{\alpha} & 0 & 0 \\
\beta & \alpha & 0 \\
0 & i \beta & \alpha-\bar{\alpha}
\end{array}\right),
\end{aligned}
$$

in terms of the group-invariant 1 -forms

$$
\begin{aligned}
& \alpha:=\frac{d \mathrm{c}}{\mathrm{c}}, \\
& \beta:=i \frac{\mathrm{e} d \mathrm{c}}{\mathrm{cc}}-i \frac{\mathrm{e} d \overline{\mathrm{c}}}{\mathrm{c} \overline{\mathrm{c}}}-i \frac{d \mathrm{e}}{\mathrm{c}} .
\end{aligned}
$$

Now, if we exterior-differentiate the lifted coframe on the product manifold equipped with coordinates

$$
\left(z_{1}, z_{2}, \bar{z}_{1}, \bar{z}_{2}, v\right) \times(\mathrm{c}, \overline{\mathrm{c}}, \mathrm{e}, \overline{\mathrm{e}}) \in M^{5} \times G^{4}
$$

after hard computations, we may come to structure equations of the abstract shape

$$
\begin{aligned}
d \rho= & (\alpha+\bar{\alpha}) \wedge \rho+R^{1} \rho \wedge \kappa+R^{2} \rho \wedge \zeta+\bar{R}^{1} \rho \wedge \bar{\kappa}+\bar{R}^{2} \rho \wedge \bar{\zeta}+i \kappa \wedge \bar{\kappa}, \\
d \kappa= & \beta \wedge \rho+\alpha \wedge \kappa+K^{1} \rho \wedge \kappa+K^{2} \rho \wedge \zeta+K^{3} \rho \wedge \bar{\kappa}+K^{4} \rho \wedge \bar{\zeta} \\
& +K^{5} \kappa \wedge \zeta+K^{6} \kappa \wedge \bar{\kappa}+\zeta \wedge \bar{\kappa}, \\
d \zeta= & \gamma \wedge \rho+i \beta \wedge \kappa+(\alpha-\bar{\alpha}) \wedge \zeta \\
& +Z^{1} \rho \wedge \kappa+Z^{2} \rho \wedge \zeta+Z^{3} \rho \wedge \bar{\kappa}+Z^{4} \rho \wedge \bar{\zeta} \\
& +Z^{5} \kappa \wedge \zeta+Z^{6} \kappa \wedge \bar{\kappa}+Z^{7} \kappa \wedge \bar{\zeta}+Z^{8} \zeta \wedge \bar{\kappa}+Z^{9} \zeta \wedge \bar{\zeta} .
\end{aligned}
$$

A moment of reflection convinces of the truth of

Assertion 10.2. The relations coming from the normalizations of the group parameters $f, b, c$ are preserved

$$
\begin{aligned}
& 1=[\zeta \wedge \bar{\kappa}]\{d \kappa\}, \\
& 0=\bar{R}^{1}-2 K^{6}+Z^{8}, \\
& 0=i K^{3}-Z^{6},
\end{aligned}
$$

as well as the auxiliary relations

$$
\begin{aligned}
K^{5} & =R^{2}, \\
Z^{7} & =i K^{4}, \\
Z^{9} & =-\bar{R}^{2} .
\end{aligned}
$$


Now, we want to absorb as many as possible of these torsion coefficients. So we introduce modified Maurer-Cartan forms - mind notations

$$
\begin{aligned}
& \pi^{1}:=\alpha-a_{1} \rho-a_{2} \kappa-a_{3} \zeta-a_{4} \bar{\kappa}-a_{5} \bar{\zeta} \\
& \pi^{2}:=\beta-b_{1} \rho-b_{2} \kappa-b_{3} \zeta-b_{4} \bar{\kappa}-b_{5} \bar{\zeta}
\end{aligned}
$$

and we try to determine (fix) the unknown coefficients $a_{i}, b_{i}$. By replacement, setting $c_{i}:=0$ in the formula seen above for $d \zeta$, we obtain without pain

$$
\begin{aligned}
d \rho= & \left(\pi^{1}+\bar{\pi}^{1}\right)+\rho \wedge \kappa\left(R^{1}-a_{2}-\bar{a}_{4}\right)+\rho \wedge \zeta\left(R^{2}-a_{3}-\bar{a}_{5}\right) \\
& +\rho \wedge \bar{\kappa}\left(\bar{R}^{1}-a_{4}-\bar{a}_{2}\right)+\rho \wedge \bar{\zeta}\left(\bar{R}^{2}-a_{5}-\bar{a}_{3}\right)+i \kappa \wedge \bar{\kappa}, \\
d \kappa= & \pi^{2} \wedge \rho+\pi^{1} \wedge \kappa+\rho \wedge \kappa\left(K^{1}+a_{1}-b_{2}\right)+\rho \wedge \zeta\left(K^{2}-b_{3}\right) \\
& +\rho \wedge \bar{\kappa}\left(K^{3}-b_{4}\right)+\rho \wedge \bar{\zeta}\left(K^{4}-b_{5}\right)+\kappa \wedge \zeta\left(K^{5}-a_{3}\right) \\
& +\kappa \wedge \bar{\kappa}\left(K^{6}-a_{4}\right)+\kappa \wedge \bar{\zeta}\left(-a_{5}\right)+\zeta \wedge \bar{\kappa}, \\
d \zeta= & i \pi^{2} \wedge \kappa+\left(\pi^{1}-\bar{\pi}^{1}\right) \wedge \zeta+\rho \wedge \kappa\left(Z^{1}+i b_{1}\right)+\rho \wedge \zeta\left(Z^{2}+a_{1}-\bar{a}_{1}\right) \\
& +\rho \wedge \bar{\kappa}\left(Z^{3}\right)+\rho \wedge \bar{\zeta}\left(Z^{4}\right)+\kappa \wedge \zeta\left(Z^{5}-i b_{3}+a_{2}-\bar{a}_{4}\right)+\kappa \wedge \bar{\kappa}\left(Z^{6}-i b_{4}\right) \\
& +\kappa \wedge \bar{\zeta}\left(Z^{7}-i b_{5}\right)+\zeta \wedge \bar{\kappa}\left(Z^{8}-a_{4}+\bar{a}_{2}\right)+\zeta \wedge \bar{\zeta}\left(Z^{9}-a_{5}+\bar{a}_{3}\right) .
\end{aligned}
$$

Now, replacing from Assertion 10.2

$$
Z^{8}:=-\bar{R}^{1}+2 K^{6}, \quad Z^{6}:=i K^{3}, \quad K^{5}:=R^{2}, \quad Z^{7}:=i K^{4}, \quad Z^{9}:=-\bar{R}^{2},
$$

the absorption equations write out as

$$
\begin{aligned}
& a_{2}+\bar{a}_{4}=R^{1}, \\
& -a_{1}+b_{2}=K^{1}, \\
& i b_{1}=-Z^{1}, \\
& \underline{a_{3}+\bar{a}_{5}}=R^{2} \text {, } \\
& b_{3}=K^{2} \text {, } \\
& b_{4}=K^{3} \text {, } \\
& b_{5}=K^{4} \text {, } \\
& \underline{a_{3}}=R^{2} \text {, } \\
& a_{4}=K^{6} \text {, } \\
& \underline{-a_{5}}=0 \text {, } \\
& -a_{1}+\bar{a}_{1}=Z^{2} \text {, } \\
& 0=Z^{3} \text {, } \\
& 0=Z^{4} \text {, } \\
& -a_{2}+\bar{a}_{4}+i b_{3}=Z^{5} \text {, } \\
& i b_{4}=i K^{3} \text {, } \\
& i b_{5}=i K^{4} \text {, } \\
& -\bar{a}_{2}+a_{4}=-\bar{R}^{1}+2 K^{6}, \\
& -\bar{a}_{3}+a_{5}=-\bar{R}^{2} \text {. }
\end{aligned}
$$

The boxed $Z^{3}$ and $Z^{4}$ are clearly essential torsions, since they cannot be annihilated by any choice of $a_{i}, b_{i}$. We will compute them explicitly a bit later. 
At the end of the second colon, $a_{5}=0$, whence at the ends of the other two colons, we get $a_{3}:=R^{2}$, hence all the 4 underlined equations drop. Also, unique assignments exist for

$$
\begin{array}{ll}
b_{3}:=K^{2}, & b_{1}:=i Z^{1}, \\
b_{4}:=K^{3}, & b_{4}:=K^{3}, \\
b_{5}:=K^{4}, & b_{5}:=K^{4} \\
a_{4}:=K^{6}, &
\end{array}
$$

and it remains to solve

$$
\begin{aligned}
& a_{2}+\bar{K}^{6} \quad \stackrel{*}{=} R^{1},-a_{1}+b_{2}=K^{1},-a_{1}+\bar{a}_{1}=Z^{2}, \\
&-a_{2}+\bar{K}^{6}+i K^{2} \stackrel{?}{=} Z^{5}, \\
&-\bar{a}_{2}+K^{6} \stackrel{*}{=}-\bar{R}^{1}+2 K^{6} .
\end{aligned}
$$

Certainly

$$
b_{2}:=K^{1}+a_{1}
$$

and the two equations $\stackrel{*}{=}$ for $a_{2}$ are equivalent - this comes from the normalization relation $0=\bar{R}^{1}-2 K^{6}+Z^{8}$ already taken account of - , yielding

$$
a_{2}:=R^{1}-\bar{K}^{6} \text {. }
$$

However, the equation $\stackrel{?}{=}$ cannot be satisfied automatically, and this provides an essential torsion combination

$$
-R^{1}+\bar{K}^{6}+\bar{K}^{6}+i K^{2}=Z^{5} \quad \Longleftrightarrow \quad-i K^{2}+Z^{5}-\bar{Z}^{8}=0,
$$

which was already seen in Lemma 8.6. The last remaining equation

$$
-a_{1}+\bar{a}_{1}=Z^{2}
$$

shows that one can annihilate $\operatorname{Im} Z^{2}$ by choosing

$$
\operatorname{Im} a_{1}:=-\frac{1}{2} \operatorname{Im} Z^{2}
$$

and it still remains precisely one real degree of freedom, a free variable that we will re-denote

$$
\mathrm{t}:=\operatorname{Re} a_{1} .
$$

In summary, we have established a fundamental

Proposition 10.3. With $\mathrm{t} \in \mathbb{R}$ being a free variable, by defining the precise modified Maurer-Cartan forms

it holds

$$
\begin{aligned}
& \pi^{1}:=\alpha-\left(\mathrm{t}-\frac{i}{2} \operatorname{Im} Z^{2}\right) \rho-\left(R^{1}-\bar{K}^{6}\right) \kappa-R^{2} \zeta-K^{6} \bar{\kappa}-0 \\
& \pi^{2}:=\beta-i Z^{1} \rho-\left(\mathrm{t}-\frac{i}{2} \operatorname{Im} Z^{2}+K^{1}\right) \kappa-K^{2} \zeta-K^{3} \bar{\kappa}-K^{4} \bar{\zeta}
\end{aligned}
$$

$$
\begin{aligned}
d \rho= & \left(\pi^{1}+\bar{\pi}^{1}\right) \wedge \rho+i \kappa \wedge \bar{\kappa} \\
d \kappa= & \pi^{2} \wedge \rho+\pi^{1} \wedge \kappa+\zeta \wedge \bar{\kappa} \\
d \zeta= & \left(\pi^{1}-\bar{\pi}^{1}\right) \wedge \zeta+i \pi^{2} \wedge \kappa \\
& +\left(\operatorname{Re} Z^{2}\right) \rho \wedge \zeta+Z^{3} \rho \wedge \bar{\kappa}+Z^{4} \rho \wedge \bar{\zeta}+\left(Z^{5}+R^{1}-2 \bar{K}^{6}-i K^{2}\right) \kappa \wedge \zeta .
\end{aligned}
$$


We yet have to compute the remaining 4 essential torsion coefficients

$$
\operatorname{Re} Z^{2}, \quad Z^{3}, \quad Z^{4}, \quad Z^{5}+R^{1}-2 \bar{K}^{6}-i K^{2} .
$$

Fortunately, by anticipation, we have already explored and finalized

$$
\begin{aligned}
Z^{5}+R^{1}-2 \bar{K}^{6}-i K^{2} & =-i K^{2}+Z^{5}-\bar{Z}^{8} \\
& =\frac{1}{c} W_{0} .
\end{aligned}
$$

Assertion 10.4. One torsion coefficient vanishes identically

$$
0 \equiv Z^{4} \text {. }
$$

Proof. Recall

$$
\begin{aligned}
Z^{4} & =[\rho \wedge \bar{\zeta}]\{d \zeta\} \\
& =[\rho \wedge \bar{\zeta}]\left\{-\frac{i}{2} \frac{\bar{c} \text { ee }}{\mathrm{c}} d \rho_{0}+\mathrm{e} d \kappa_{0}^{\prime}+\frac{\mathrm{c}}{\overline{\mathrm{c}}} d \zeta_{0}^{\prime \prime}\right\}
\end{aligned}
$$

Compute separately

$$
\begin{aligned}
& -\frac{i}{2} \frac{\overline{\mathrm{cee}}}{\mathrm{c}}[\rho \wedge \bar{\zeta}]\left\{d \rho_{0}\right\}=-\frac{i}{2} \frac{\mathrm{cee}}{\mathrm{c}} \bar{R}_{0}^{2 \prime}\left(\frac{1}{\mathrm{c} \overline{\mathrm{C}}}\right)\left(\begin{array}{l}
\mathrm{c} \\
\overline{\mathrm{c}}
\end{array}\right)=-\frac{i}{2} \frac{\mathrm{ee}}{\mathrm{c \overline { \textrm {c } }}} \bar{R}_{0}^{2 \prime}, \\
& \mathrm{e}[\rho \wedge \bar{\zeta}]\left\{d \kappa_{0}^{\prime}\right\}=0, \\
& \frac{\mathrm{c}}{\overline{\mathrm{c}}}[\rho \wedge \bar{\zeta}]\left\{d \zeta_{0}^{\prime \prime}\right\}=\frac{\mathrm{c}}{\overline{\mathrm{c}}} \boldsymbol{Z}_{0}^{4 \prime \prime}{ }_{\circ}\left(\frac{1}{\mathrm{c} \overline{\mathrm{c}}}\right)\left(\begin{array}{l}
\mathrm{c} \\
\overline{\mathrm{c}}
\end{array}\right)+\frac{\mathrm{c}}{\overline{\mathrm{c}}} \boldsymbol{Z}_{0}^{9 \prime}\left(-\frac{i}{2} \frac{\overline{\mathrm{cee}}}{\mathrm{ccc}}\right)\left(\frac{\mathrm{c}}{\overline{\mathrm{c}}}\right) \\
& =0-\frac{i}{2} \frac{\mathrm{ee}}{\mathrm{cc}} Z_{0}^{9 \prime}
\end{aligned}
$$

and since we have already seen in Lemma 9.1 that $Z_{0}^{4 \prime \prime} \equiv 0$, in the proof of which we have used $\bar{R}_{0}^{2 \prime}+Z_{0}^{9 \prime} \equiv 0$, the sum of these 3 terms is indeed zero, and we done.

It remains to analyze $Z^{3}$ and $\operatorname{Re} Z^{2}$, a substantial task to which the two next sections are devoted. At least, we know that

$$
\begin{aligned}
d \zeta= & \left(\pi^{1}-\bar{\pi}^{1}\right) \wedge \rho+i \kappa \wedge \bar{\kappa} \\
& +\left(\operatorname{Re} Z^{2}\right) \rho \wedge \zeta+Z^{3} \rho \wedge \bar{\kappa}+\frac{1}{c} W_{0} \kappa \wedge \zeta .
\end{aligned}
$$

\section{COMPUtATION OF POCCHIOLA'S INVARIANT $\boldsymbol{J}_{0}$}

We now determine

$$
\begin{aligned}
Z^{3}= & {[\rho \wedge \bar{\kappa}]\{d \zeta\} } \\
= & -\frac{i}{2} \frac{\overline{\mathrm{cee}}}{\mathrm{c}}[\rho \wedge \bar{\kappa}]\left\{d \rho_{0}\right\}+\mathrm{e}[\rho \wedge \bar{\kappa}]\left\{d \kappa_{0}^{\prime}\right\}+\frac{\mathrm{c}}{\overline{\mathrm{c}}}[\rho \wedge \bar{\kappa}]\left\{d \zeta_{0}^{\prime \prime}\right\} \\
= & -\frac{i}{2} \frac{\overline{\mathrm{cee}}}{\mathrm{c}}\left[\bar{R}_{0}^{1 \prime}\left(\frac{1}{\mathrm{c} \overline{\mathrm{c}}}\right)\left(\frac{1}{\overline{\mathrm{c}}}\right)+\bar{R}_{0}^{2 \prime}\left(\frac{1}{\mathrm{c \overline {c }}}\right)\left(-\frac{\mathrm{c} \overline{\overline{\mathrm{c}}}}{\overline{\mathrm{cc}}}\right)+i\left(i \frac{\mathrm{e}}{\mathrm{cc}}\right)\left(\frac{1}{\overline{\mathrm{c}}}\right)_{\circ}\right] \\
& +\mathrm{e}\left[K_{0}^{3 \prime \prime}\left(\frac{1}{\mathrm{c \overline {c }}}\right)\left(\frac{1}{\overline{\mathrm{c}}}\right)+K_{0}^{6 \prime}\left(i \frac{\mathrm{e}}{\mathrm{cc}}\right)\left(\frac{1}{\overline{\mathrm{c}}}\right)+\left(-\frac{i}{\frac{\mathrm{cee}}{\mathrm{ccc}}}\right)\left(\frac{1}{\overline{\mathrm{c}}}\right)_{\circ}\right] \\
& +\frac{\mathrm{c}}{\overline{\mathrm{c}}}\left[Z_{0}^{3 \prime \prime}\left(\frac{1}{\mathrm{c \overline {c }}}\right)\left(\frac{1}{\overline{\mathrm{c}}}\right)+Z_{0}^{6 \prime \prime}\left(i \frac{\mathrm{e}}{\mathrm{cc}}\right)\left(\frac{1}{\overline{\mathrm{c}}}\right)+Z_{0}^{8 \prime}\left(-\frac{i}{2} \frac{\overline{\mathrm{cee}}}{\mathrm{ccc}}\right)\left(\frac{1}{\overline{\mathrm{c}}}\right)+Z_{0}^{9 \prime}\left(-\frac{i}{2} \frac{\overline{\mathrm{cee}}}{\mathrm{ccc}}\right)\left(\frac{\overline{\mathrm{ce}}}{\overline{\mathrm{cc}}}\right)\right],
\end{aligned}
$$


hence after collecting

$$
\begin{aligned}
Z^{3}= & \frac{\mathrm{ee}}{\mathrm{cc} \overline{\mathrm{C}}}\left[-\frac{i}{2} \bar{R}_{0}^{1 \prime}+i K_{0}^{6 \prime}-\frac{i}{2} \boldsymbol{Z}_{0}^{8 \prime}\right]+\frac{\mathrm{ee} \overline{\mathrm{e}}}{\mathrm{c}}\left[\frac{i}{2} \bar{R}_{0}^{2 \prime}+\frac{i}{2} \boldsymbol{Z}_{0}^{9 \prime}\right] \\
& +\frac{\mathrm{e}}{\mathrm{c} \overline{\mathrm{CC}}}\left[K_{0}^{3 \prime \prime}+i \boldsymbol{Z}_{0}^{6 \prime \prime}\right]+\frac{1}{\overline{\mathrm{cCC}}} \boldsymbol{Z}_{0}^{3 \prime \prime} .
\end{aligned}
$$

As we already know, the second term vanishes, the third one as well

$$
K_{0}^{3 \prime \prime}+i Z_{0}^{6 \prime \prime}=2 i H_{0}-i H_{0}-i H_{0}
$$

and also the first one

$$
\begin{aligned}
-\frac{i}{2} \overline{\boldsymbol{R}}_{0}^{1 \prime}+i \boldsymbol{K}_{0}^{6 \prime}-\frac{i}{2} \boldsymbol{Z}_{0}^{8 \prime}= & -\frac{i}{2}\left(\frac{1}{3} \frac{\overline{\mathcal{L}}_{1}\left(\overline{\mathcal{L}}_{1}(k)\right)}{\overline{\mathcal{L}}_{1}(k)}+\frac{2}{3} \bar{P}\right)+i\left(-\frac{1}{3} \frac{\overline{\mathcal{L}}_{1}\left(\overline{\mathcal{L}}_{1}(k)\right)}{\overline{\mathcal{L}}_{1}(k)}+\frac{1}{3} \bar{P}\right) \\
& -\frac{i}{2}\left(-\frac{\overline{\mathcal{L}}_{1}\left(\overline{\mathcal{L}}_{1}(k)\right)}{\overline{\mathcal{L}}_{1}(k)}\right) .
\end{aligned}
$$

It remains only one term

$$
\begin{aligned}
Z^{3} & =\frac{1}{\overline{\mathrm{CCC}}} Z_{0}^{3 \prime \prime} \\
& =\frac{1}{\overline{\mathrm{CCC}}}\left(-i \boldsymbol{Z}_{0}^{8 \prime} H_{0}-i \overline{\mathcal{L}}_{1}\left(H_{0}\right)+i H_{0} \bar{R}_{0}^{1 \prime}\right) \\
& =\frac{i}{\overline{\mathrm{CCC}}}\left(\frac{\overline{\mathcal{L}}_{1}\left(\overline{\mathcal{L}}_{1}(k)\right)}{\overline{\mathcal{L}}_{1}(k)} H_{0}-\overline{\mathcal{L}}_{1}\left(H_{0}\right)+\frac{1}{3} \frac{\overline{\mathcal{L}}_{1}\left(\overline{\mathcal{L}}_{1}(k)\right)}{\overline{\mathcal{L}}_{1}(k)} H_{0}+\frac{2}{3} H_{0} \bar{P}\right) \\
& =\frac{i}{\overline{\mathrm{CCC}}}(\underbrace{\frac{4}{3} \frac{\overline{\mathcal{L}}_{1}\left(\overline{\mathcal{L}}_{1}(k)\right)}{\overline{\mathcal{L}}_{1}(k)} H_{0}+\frac{2}{3} \bar{P}_{0} H_{0}-\overline{\mathcal{L}}_{1}\left(H_{0}\right)}_{=: \bar{J}_{0}}) .
\end{aligned}
$$

Then a direct expansion of the derivative $\overline{\mathcal{L}}_{1}\left(H_{0}\right)$ which uses neither Lemma 2.5, nor Lemma 2.9, provides (exercise) exactly the same expression as the one of Pocchiola

$$
\begin{aligned}
\bar{J}_{0}= & \frac{1}{6} \frac{\overline{\mathcal{L}}_{1}\left(\overline{\mathcal{L}}_{1}\left(\overline{\mathcal{L}}_{1}\left(\overline{\mathcal{L}}_{1}(k)\right)\right)\right)}{\overline{\mathcal{L}}_{1}(k)}-\frac{5}{6} \frac{\overline{\mathcal{L}}_{1}\left(\overline{\mathcal{L}}_{1}\left(\overline{\mathcal{L}}_{1}(k)\right)\right) \overline{\mathcal{L}}_{1}\left(\overline{\mathcal{L}}_{1}(k)\right)}{\overline{\mathcal{L}}_{1}(k)^{2}}-\frac{1}{6} \frac{\overline{\mathcal{L}}_{1}\left(\overline{\mathcal{L}}_{1}\left(\overline{\mathcal{L}}_{1}(k)\right)\right)}{\overline{\mathcal{L}}_{1}(k)} \bar{P} \\
& +\frac{20}{27} \frac{\overline{\mathcal{L}}_{1}\left(\overline{\mathcal{L}}_{1}(k)\right)^{3}}{\overline{\mathcal{L}}_{1}(k)^{3}}+\frac{5}{18} \frac{\overline{\mathcal{L}}_{1}\left(\overline{\mathcal{L}}_{1}(k)\right)^{2}}{\overline{\mathcal{L}}_{1}(k)^{2}} \bar{P}+\frac{1}{6} \frac{\overline{\mathcal{L}}_{1}\left(\overline{\mathcal{L}}_{1}(k)\right) \overline{\mathcal{L}}_{1}(\bar{P})}{\overline{\mathcal{L}}_{1}(k)}-\frac{1}{9} \frac{\overline{\mathcal{L}}_{1}\left(\overline{\mathcal{L}}_{1}(k)\right)}{\overline{\mathcal{L}}_{1}(k)} \bar{P} \bar{P} \\
& -\frac{1}{6} \overline{\mathcal{L}}_{1}\left(\overline{\mathcal{L}}_{1}(\bar{P})\right)+\frac{1}{3} \overline{\mathcal{L}}_{1}(\bar{P}) \bar{P}-\frac{2}{27} \bar{P} \bar{P} \bar{P} .
\end{aligned}
$$


12. COMputation OF THE DERIVED INVARIANT $R:=\operatorname{Re} Z^{2}$

Next, we determine

$$
\begin{aligned}
Z^{2}= & {[\rho \wedge \zeta]\{d \zeta\} } \\
= & -\frac{i}{2} \frac{\overline{\mathrm{cee}}}{\mathrm{c}}[\rho \wedge \zeta]\left\{d \rho_{0}\right\}+\mathrm{e}[\rho \wedge \zeta]\left\{d \kappa_{0}^{\prime}\right\}+\frac{\overline{\mathrm{c}}}{\overline{\mathrm{c}}}[\rho \wedge \zeta]\left\{d \zeta_{0}^{\prime \prime}\right\} \\
= & -\frac{i}{2} \frac{\overline{\mathrm{c} e \mathrm{e}}}{\mathrm{c}}\left[R_{0}^{2 \prime}\left(\frac{1}{\mathrm{c} \overline{\mathrm{c}}}\right)\left(\frac{\overline{\mathrm{c}}}{\mathrm{c}}\right)\right] \\
& +\mathrm{e}\left[K_{0}^{2 \prime}\left(\frac{1}{\mathrm{c} \overline{\mathrm{c}}}\right)\left(\frac{\overline{\mathrm{c}}}{\mathrm{c}}\right)+K_{0}^{5 \prime}\left(i \frac{\mathrm{e}}{\mathrm{cc}}\right)\left(\frac{\overline{\mathrm{c}}}{\mathrm{c}}\right)-\left(\frac{\overline{\mathrm{c}}}{\mathrm{c}}\right)\left(-i \frac{\overline{\mathrm{e}}}{\overline{\mathrm{cC}}}\right)\right] \\
& +\frac{\mathrm{c}}{\overline{\mathrm{c}}}\left[Z_{0}^{2 \prime \prime}\left(\frac{1}{\mathrm{c} \overline{\mathrm{c}}}\right)\left(\frac{\overline{\mathrm{c}}}{\mathrm{c}}\right)+Z_{0}^{5 \prime}\left(i \frac{\mathrm{e}}{\mathrm{cc}}\right)\left(\frac{\overline{\mathrm{c}}}{\mathrm{c}}\right)-Z_{0}^{8 \prime}\left(\frac{\overline{\mathrm{c}}}{\mathrm{c}}\right)\left(-i \frac{\overline{\mathrm{e}}}{\overline{\mathrm{cc}}}\right)-Z_{0}^{9 \prime}\left(\frac{\overline{\mathrm{c}}}{\mathrm{c}}\right)\left(\frac{i}{2} \frac{\mathrm{c} \overline{\overline{\mathrm{CC}}}}{\overline{\mathrm{cc}}}\right)\right]
\end{aligned}
$$

hence after collecting

$$
\begin{aligned}
Z^{2}= & i \frac{\mathrm{e} \overline{\mathrm{e}}}{\mathrm{c} \overline{\mathrm{c}}}+\frac{\overline{\mathrm{cee}}}{\mathrm{ccc}}\left(-\frac{i}{2} R_{0}^{2 \prime}+i \boldsymbol{K}_{0}^{5 \prime}\right)+\frac{\mathrm{c} \overline{\mathrm{e}}}{\overline{\mathrm{ccc}}}\left(-\frac{i}{2} Z_{0}^{9 \prime}\right) \\
& +\frac{\mathrm{e}}{\mathrm{cc}}\left(K_{0}^{2 \prime}+i \boldsymbol{Z}_{0}^{5 \prime}\right)+\frac{\overline{\mathrm{e}}}{\overline{\mathrm{cc}}}\left(i \boldsymbol{Z}_{0}^{8 \prime}\right)+\frac{1}{\mathrm{c} \overline{\mathrm{c}}} \boldsymbol{Z}_{0}^{2 \prime \prime}
\end{aligned}
$$

that is to say

$$
\begin{aligned}
Z^{2}= & i \frac{\mathrm{ee}}{\overline{\mathrm{cc}}}+\frac{\overline{\mathrm{cee}}}{\mathrm{ccc}}\left(\frac{i}{2} \frac{\mathcal{L}_{1}(k)}{\overline{\mathcal{L}}_{1}(k)}-i \frac{\mathcal{L}_{1}(k)}{\overline{\mathcal{L}}_{1}(k)}\right)+\frac{\mathrm{cee}}{\overline{\mathrm{ccC}}}\left(-\frac{i}{2} \frac{\overline{\mathcal{L}}_{1}(\bar{k})}{\mathcal{L}_{1}(\bar{k})}\right) \\
& +\frac{\mathrm{e}}{\mathrm{cc}}\left(-\frac{i}{3} \frac{\mathcal{K}\left(\overline{\mathcal{L}}_{1}\left(\overline{\mathcal{L}}_{1}(k)\right)\right)}{\overline{\mathcal{L}}_{1}(k)^{2}}+\frac{i}{3} \frac{\mathcal{K}\left(\overline{\mathcal{L}}_{1}(k)\right) \overline{\mathcal{L}}_{1}\left(\overline{\mathcal{L}}_{1}(k)\right)}{\overline{\mathcal{L}}_{1}(k)^{3}}-\frac{i}{3} \frac{\mathcal{L}_{1}\left(\mathcal{L}_{1}(\bar{k})\right)}{\mathcal{L}_{1}(\bar{k})}\right. \\
& \left.-\frac{i}{3} \frac{\overline{\mathcal{L}}_{1}\left(\mathcal{L}_{1}(k)\right)}{\overline{\mathcal{L}}_{1}(k)}-\frac{2}{3} \frac{\mathcal{T}(k)}{\overline{\mathcal{L}}_{1}(k)}+i \frac{\mathcal{L}_{1}\left(\overline{\mathcal{L}}_{1}(k)\right)}{\overline{\mathcal{L}}_{1}(k)}\right) \\
& +\frac{\overline{\mathrm{e}}}{\overline{\mathrm{cC}}}\left(-i \frac{\overline{\mathcal{L}}_{1}\left(\overline{\mathcal{L}}_{1}(k)\right)}{\overline{\mathcal{L}}_{1}(k)}\right)+\frac{1}{\mathrm{c} \overline{\mathrm{c}}}(\underbrace{-i Z_{0}^{9 \prime} \bar{H}_{0}+i H_{0} R_{0}^{2 \prime}}_{\text {on hold }}+Z_{0}^{2 \prime}-i \frac{\mathcal{K}\left(H_{0}\right)}{\overline{\mathcal{L}}_{1}(k)}) .
\end{aligned}
$$

Now, observe firstly that when we consider

$$
2 \operatorname{Re} Z^{2}=Z^{2}+\bar{Z}^{2},
$$

the real part of the sum of the first three terms of $Z^{2}$

$$
i \frac{\mathrm{e} \overline{\mathrm{e}}}{\mathrm{c} \overline{\mathrm{C}}}+\frac{\overline{\mathrm{cee}}}{\mathrm{ccc}}\left(-\frac{i}{2} \frac{\mathcal{L}_{1}(k)}{\overline{\mathcal{L}}_{1}(k)}\right)+\frac{\mathrm{c} \overline{\mathrm{ee}}}{\overline{\mathrm{ccC}}}\left(-\frac{i}{2} \frac{\overline{\mathcal{L}}_{1}(\bar{k})}{\mathcal{L}_{1}(\bar{k})}\right)
$$


vanishes, visibly. Secondly, in the sum $Z^{2}+\bar{Z}^{2}$, if the terms multiples of $\frac{\mathrm{e}}{\mathrm{cc}}$ are grouped together, we realize that we recover $W_{0}$ exactly

$$
\begin{aligned}
i \frac{\mathrm{e}}{\mathrm{cc}}( & -\frac{1}{3} \frac{\mathcal{K}\left(\overline{\mathcal{L}}_{1}\left(\overline{\mathcal{L}}_{1}(k)\right)\right)}{\overline{\mathcal{L}}_{1}(k)^{2}}+\frac{1}{3} \frac{\mathcal{K}\left(\overline{\mathcal{L}}_{1}(k)\right) \overline{\mathcal{L}}_{1}\left(\overline{\mathcal{L}}_{1}(k)\right)}{\overline{\mathcal{L}}_{1}(k)^{3}}-\frac{1}{3} \frac{\mathcal{L}_{1}\left(\mathcal{L}_{1}(\bar{k})\right)}{\mathcal{L}_{1}(\bar{k})} \\
& \left.-\frac{1}{3} \frac{\overline{\mathcal{L}}_{1}\left(\mathcal{L}_{1}(k)\right)}{\overline{\mathcal{L}}_{1}(k)}+\frac{2 i}{3} \frac{\mathcal{T}(k)}{\overline{\mathcal{L}}_{1}(k)}+\frac{\mathcal{L}_{1}\left(\overline{\mathcal{L}}_{1}(k)\right)}{\overline{\mathcal{L}}_{1}(k)}+\frac{\mathcal{L}_{1}\left(\mathcal{L}_{1}(\bar{k})\right)}{\mathcal{L}_{1}(\bar{k})}\right) \\
= & i \frac{\mathrm{e}}{\mathrm{cc}}\left(-\frac{1}{3} \frac{\mathcal{K}\left(\overline{\mathcal{L}}_{1}\left(\overline{\mathcal{L}}_{1}(k)\right)\right)}{\overline{\mathcal{L}}_{1}(k)^{2}}+\frac{1}{3} \frac{\mathcal{K}\left(\overline{\mathcal{L}}_{1}(k)\right) \overline{\mathcal{L}}_{1}\left(\overline{\mathcal{L}}_{1}(k)\right)}{\overline{\mathcal{L}}_{1}(k)^{3}}+\frac{2}{3} \frac{\mathcal{L}_{1}\left(\mathcal{L}_{1}(\bar{k})\right)}{\mathcal{L}_{1}(\bar{k})}\right. \\
& \left.+\frac{2}{3} \frac{\mathcal{L}_{1}\left(\overline{\mathcal{L}}_{1}(k)\right)}{\overline{\mathcal{L}}_{1}(k)}+\frac{i}{3} \frac{\mathcal{T}(k)}{\overline{\mathcal{L}}_{1}(k)}\right) \\
= & i \frac{\mathrm{e}}{\mathrm{cc}} W_{0},
\end{aligned}
$$

as we remember its explicit expression from Section 8 .

In addition thirdly, using the explicit expressions from Proposition 7.2

$$
R_{0}^{2 \prime}=-\frac{\mathcal{L}_{1}(k)}{\overline{\mathcal{L}}_{1}(k)} \quad \text { and } \quad Z_{0}^{9 \prime}=\frac{\overline{\mathcal{L}}_{1}(\bar{k})}{\mathcal{L}_{1}(\bar{k})},
$$

and the explicit expression of

$$
H_{0}=-\frac{1}{6} \frac{\overline{\mathcal{L}}_{1}\left(\overline{\mathcal{L}}_{1}\left(\overline{\mathcal{L}}_{1}(k)\right)\right)}{\overline{\mathcal{L}}_{1}(k)}+\frac{2}{9} \frac{\overline{\mathcal{L}}_{1}\left(\overline{\mathcal{L}}_{1}(k)\right)^{2}}{\overline{\mathcal{L}}_{1}(k)^{2}}+\frac{1}{18} \frac{\overline{\mathcal{L}}_{1}\left(\overline{\mathcal{L}}_{1}(k)\right)}{\overline{\mathcal{L}}_{1}(k)} \bar{P}+\frac{1}{6} \overline{\mathcal{L}}_{1}(\overline{\boldsymbol{P}})-\frac{1}{9} \bar{P} \bar{P},
$$

we verify by a direct computation the identical vanishing

$$
0 \equiv-i Z_{0}^{9 \prime} \bar{H}_{0}+i H_{0} R_{0}^{2 \prime}+\overline{-i Z_{0}^{9 \prime} \bar{H}_{0}+i H_{0} R_{0}^{2 \prime}},
$$

which means that the term 'on hold' underbraced above disappears when taking $2 \operatorname{Re} Z^{2}$, and we receive

$$
2 \operatorname{Re} Z^{2}=i \frac{\mathrm{e}}{\mathrm{cC}} W_{0}-i \frac{\overline{\mathrm{e}}}{\overline{\mathrm{CC}}} \bar{W}_{0}+\frac{1}{\mathrm{c} \overline{\mathrm{C}}}\left(Z_{0}^{2 \prime}-i \frac{\mathcal{K}\left(H_{0}\right)}{\overline{\mathcal{L}}_{1}(k)}+\bar{Z}_{0}^{2 \prime}+i \frac{\overline{\mathcal{K}}\left(\bar{H}_{0}\right)}{\mathcal{L}_{1}(\bar{k})}\right) .
$$

Fourthly and lastly, by replacing

$$
H_{0}=-\frac{i}{2} K_{0}^{3 \prime}
$$

we get

$$
2 \operatorname{Re} Z^{2}=2 \operatorname{Re}\left(i \frac{\mathrm{e}}{\mathrm{cc}} W_{0}+\frac{1}{\mathrm{cc}}(Z_{0}^{2 \prime} \underbrace{-\frac{1}{2} \frac{\mathcal{K}\left(K_{0}^{3 \prime}\right)}{\overline{\mathcal{L}}_{1}(k)}}_{\text {on hold }})\right) .
$$

A miraculous re-expression of $2 \operatorname{Re} Z^{2}$ was discovered by Pocchiola on his computer, and was shown in $[25,18]$, but without any details of proof.

Lemma 12.2. One has in fact

$$
2 \operatorname{Re} Z^{2}=2 \operatorname{Re}\left[i \frac{\mathrm{e}}{\mathrm{cC}} W_{0}+\frac{1}{\mathrm{cc}}\left(-\frac{i}{2} \overline{\mathcal{L}}_{1}\left(W_{0}\right)+\frac{i}{2}\left(-\frac{1}{3} \frac{\overline{\mathcal{L}}_{1}\left(\overline{\mathcal{L}}_{1}(k)\right)}{\overline{\mathcal{L}}_{1}(k)}+\frac{1}{3} \bar{P}\right) W_{0}\right)\right] .
$$


This expression shows that $\operatorname{Re}\left(Z^{2}\right)$ depends on the first jet of $W_{0}$, that it vanishes when $W_{0}=0$, and therefore, $\operatorname{Re} Z^{2}$ is not a primary invariant. We provide details of proof, with no computer help.

Proof. To transform the term 'on hold' above, we need a technical lemma, whose proof, to be done afterwards, uses mainly the Poincaré relation $d \circ d=0$ applied to the structure equations (7.1).

Lemma 12.3. The following two identities hold identically

$$
\begin{aligned}
\frac{\mathcal{K}\left(K_{0}^{3 \prime}\right)}{\overline{\mathcal{L}}_{1}(k)} & =\overline{\mathcal{L}}_{1}\left(K_{0}^{2 \prime}\right)-K_{0}^{2 \prime} K_{0}^{6 \prime}-K_{0}^{1 \prime}+\bar{K}_{0}^{1 \prime}+Z_{0}^{2 \prime}, \\
\overline{\mathcal{L}}_{1}\left(Z_{0}^{5 \prime}\right)+\mathcal{L}_{1}\left(Z_{0}^{8 \prime}\right) & =Z_{0}^{5 \prime} K_{0}^{6 \prime}+Z_{0}^{8 \prime} \bar{K}_{0}^{6 \prime}+i Z_{0}^{2 \prime} .
\end{aligned}
$$

Admitting these identities temporarily, let us prove the proposition. In order to replace the term 'on hold' in (12.1) above, let us multiply by $-\frac{1}{2}$ the first identity (12.4), and take $2 \operatorname{Re}(\bullet)$

$$
2 \operatorname{Re}\left(-\frac{1}{2} \frac{\mathcal{K}\left(K_{0}^{3 \prime}\right)}{\overline{\mathcal{L}}_{1}(k)}\right)=2 \operatorname{Re}\left(-\frac{1}{2} \overline{\mathcal{L}}_{1}\left(K_{0}^{2 \prime}\right)+\frac{1}{2} K_{0}^{2 \prime} K_{0}^{6 \prime}+0-\frac{1}{2} Z_{0}^{2 \prime}\right) .
$$

We yet have to transform the boxed term. To this aim, we conjugate the second identity (12.5)

$$
\mathcal{L}_{1}\left(\bar{Z}_{0}^{5 \prime}\right)+\overline{\mathcal{L}}_{1}\left(\bar{Z}_{0}^{8 \prime}\right)=\bar{Z}_{0}^{5 \prime} \bar{K}_{0}^{6 \prime}+\bar{Z}_{0}^{8 \prime} K_{0}^{6 \prime}-i \bar{Z}_{0}^{2 \prime},
$$

and to this identity multiplied by $i$, we subtract (12.4) also multiplied by $i$, to get

$$
-i \overline{\mathcal{L}}_{1}\left(Z_{0}^{5 \prime}-\bar{Z}_{0}^{8 \prime}\right)+i \mathcal{L}_{1}\left(\bar{Z}_{0}^{5 \prime}-Z_{0}^{8 \prime}\right)=-i K_{0}^{6 \prime}\left(Z_{0}^{5 \prime}-\bar{Z}_{0}^{8 \prime}\right)+i \bar{K}_{0}^{6 \prime}\left(\bar{Z}_{0}^{5 \prime}-Z_{0}^{8 \prime}\right)+Z_{0}^{2 \prime}+\bar{Z}_{0}^{2 \prime} .
$$

But here, remembering that, by definition of $W_{0}$

$$
Z_{0}^{5 \prime}-\bar{Z}_{0}^{8 \prime}=W_{0}+i K_{0}^{2 \prime}
$$

we can replace to get

$-i \overline{\mathcal{L}}_{1}\left(W_{0}\right)+\overline{\mathcal{L}}_{1}\left(K_{0}^{2 \prime}\right)+i \mathcal{L}_{1}\left(\bar{W}_{0}\right)+\mathcal{L}_{1}\left(\bar{K}_{0}^{2 \prime}\right)=-i K_{0}^{6 \prime} W_{0}+K_{0}^{6 \prime} K_{0}^{2 \prime}+i \bar{K}_{0}^{6 \prime} \bar{W}_{0}+\bar{K}_{0}^{6 \prime} \bar{K}_{0}^{2 \prime}+Z_{0}^{2 \prime}+\bar{Z}_{0}^{2 \prime}$, that is to say for the mentioned boxed term

$$
2 \operatorname{Re}\left(\overline{\mathcal{L}}_{1}\left(K_{0}^{2 \prime}\right)\right)=2 \operatorname{Re}\left(i \overline{\mathcal{L}}_{1}\left(W_{0}\right)-i K_{0}^{6 \prime} W_{0}+K_{0}^{2 \prime} K_{0}^{6 \prime}+Z_{0}^{2 \prime}\right) .
$$

Multiplying this result by $-\frac{1}{2}$, and replacing above yields

$2 \operatorname{Re}\left(-\frac{1}{2} \frac{\mathcal{K}\left(K_{0}^{3 \prime}\right)}{\overline{\mathcal{L}}_{1}(k)}\right)=2 \operatorname{Re}\left(-\frac{i}{2} \overline{\mathcal{L}}_{1}\left(W_{0}\right)+\frac{i}{2} K_{0}^{6 \prime} W_{0}-\underline{\frac{1}{2}} K_{0}^{2 \prime} K_{0}^{6 \prime}-\frac{1}{2} Z_{0}^{2 \prime}+\underline{\frac{1}{2}} K_{0}^{2 \prime} K_{0}^{6 \prime}-\frac{1}{2} Z_{0}^{2 \prime}\right)$

and a final replacement in (12.1) concludes, if one remembers that

$$
K_{0}^{6 \prime}=-\frac{1}{3} \frac{\overline{\mathcal{L}}_{1}\left(\overline{\mathcal{L}}_{1}(k)\right)}{\overline{\mathcal{L}}_{1}(k)}+\frac{1}{3} \bar{P} .
$$


Proof of Lemma 12.3. To treat the first identity (12.4), apply the exterior differentiation operator $d$ to the structure equation for $d \kappa_{0}^{\prime}$ from (7.1)

$$
\begin{aligned}
0 & =d^{2} \kappa_{0}^{\prime} \\
& =d K_{0}^{1 \prime} \wedge \rho_{0} \wedge \kappa_{0}^{\prime}+K_{0}^{1 \prime} d \rho_{0} \wedge \kappa_{0}^{\prime}-K_{0}^{1 \prime} \rho_{0} \wedge d \kappa_{0}^{\prime} \\
& +d K_{0}^{2 \prime} \wedge \rho_{0} \wedge \zeta_{0}^{\prime}+K_{0}^{2 \prime} d \rho_{0} \wedge \zeta_{0}^{\prime}-K_{0}^{2 \prime} \rho_{0} \wedge d \zeta_{0}^{\prime} \\
& +\underbrace{d K_{0}^{3 \prime} \wedge \rho_{0} \wedge \bar{\kappa}_{0}^{\prime}}_{\text {needed }}+K_{0}^{3 \prime} d \rho_{0} \wedge \bar{\kappa}_{0}^{\prime}-K_{0}^{3 \prime} \rho_{0} \wedge d \bar{\kappa}_{0}^{\prime} \\
& +d K_{0}^{5 \prime} \wedge \kappa_{0}^{\prime} \wedge \zeta_{0}^{\prime}+K_{0}^{5 \prime} d \kappa_{0}^{\prime} \wedge \zeta_{0}^{\prime}-K_{0}^{5 \prime} \kappa_{0}^{\prime} \wedge d \zeta_{0}^{\prime} \\
& +d K_{0}^{6 \prime} \wedge \kappa_{0}^{\prime} \wedge \bar{\kappa}_{0}^{\prime}+K_{0}^{6 \prime} d \kappa_{0}^{\prime} \wedge \bar{\kappa}_{0}^{\prime}-K_{0}^{6 \prime} \kappa_{0}^{\prime} \wedge d \bar{\kappa}_{0}^{\prime} \\
& \quad+d \zeta_{0}^{\prime} \wedge \bar{\kappa}_{0}^{\prime}-\zeta_{0}^{\prime} \wedge d \bar{\kappa}_{0}^{\prime} .
\end{aligned}
$$

Because we are dealing with $\mathcal{K}\left(K_{0}^{3 \prime}\right)$, we can wedge throughout with $\kappa_{0}^{\prime} \wedge \bar{\zeta}_{0}^{\prime}$ to obtain $\mathcal{K}\left(K_{0}^{3 \prime}\right) / \overline{\mathcal{L}}_{1}(k)$ from the term marked 'needed', and we get

$$
\begin{aligned}
& 0=0 \quad+0 \quad-K_{0}^{1 \prime} \rho_{0} \wedge d \kappa_{0}^{\prime} \wedge \kappa_{0}^{\prime} \wedge \bar{\zeta}_{0}^{\prime} \\
& +d K_{0}^{2 \prime} \wedge \rho_{0} \wedge \zeta_{0}^{\prime} \wedge \kappa_{0}^{\prime} \wedge \bar{\zeta}_{0}^{\prime}+K_{0}^{2 \prime} d \rho_{0} \wedge \zeta_{0}^{\prime} \wedge \kappa_{0}^{\prime} \wedge \bar{\zeta}_{0}^{\prime}-K_{0}^{2 \prime} \rho_{0} \wedge d \zeta_{0}^{\prime} \wedge \kappa_{0}^{\prime} \wedge \bar{\zeta}_{0}^{\prime} \\
& +d \boldsymbol{K}_{0}^{3 \prime} \wedge \rho_{0} \wedge \bar{\kappa}_{0}^{\prime} \wedge \kappa_{0}^{\prime} \wedge \bar{\zeta}_{0}^{\prime}+K_{0}^{3 \prime} d \rho_{0} \wedge \bar{\kappa}_{0}^{\prime} \wedge \kappa_{0}^{\prime} \wedge \bar{\zeta}_{0}^{\prime}-K_{0}^{3 \prime} \rho_{0} \wedge d \bar{\kappa}_{0}^{\prime} \wedge \kappa_{0}^{\prime} \wedge \bar{\zeta}_{0}^{\prime} \\
& +0 \quad+K_{0}^{5 \prime} d \kappa_{0}^{\prime} \wedge \zeta_{0}^{\prime} \wedge \kappa_{0}^{\prime} \wedge \bar{\zeta}_{0}^{\prime}-0 \\
& +0 \quad+K_{0}^{6 \prime} d \kappa_{0}^{\prime} \wedge \bar{\kappa}_{0}^{\prime} \wedge \kappa_{0}^{\prime} \wedge \bar{\zeta}_{0}^{\prime}-0 \\
& +0 \quad+d \zeta_{0}^{\prime} \wedge \bar{\kappa}_{0}^{\prime} \wedge \kappa_{0}^{\prime} \wedge \bar{\zeta}_{0}^{\prime} \quad-\zeta_{0}^{\prime} \wedge d \bar{\kappa}_{0}^{\prime} \wedge \kappa_{0}^{\prime} \wedge \bar{\zeta}_{0}^{\prime} .
\end{aligned}
$$

In the left column, observe that two exterior differentials appear, $d \boldsymbol{K}_{0}^{2 \prime}, d \boldsymbol{K}_{0}^{3 \prime}$. Already in Section 9, we have implicitly used the following companion of Lemma 2.6.

Lemma 12.6. The exterior differential of any function $G=G\left(z_{1}, z_{2}, \bar{z}_{1}, \bar{z}_{2}, v\right)$ on $M$ expresses as

$$
d \boldsymbol{G}=\left(\mathcal{T}(\boldsymbol{G})-\frac{i}{3} \boldsymbol{B}_{0}+\frac{i}{3} \overline{\boldsymbol{B}}_{0}\right) \rho_{0}+\mathcal{L}_{1}(\boldsymbol{G}) \kappa_{0}^{\prime}+\frac{\mathcal{K}(\boldsymbol{G})}{\overline{\mathcal{L}}_{1}(k)} \zeta_{0}^{\prime}+\overline{\mathcal{L}}_{1}(\boldsymbol{G}) \bar{\kappa}_{0}^{\prime}+\frac{\mathcal{K}(\boldsymbol{G})}{\mathcal{L}_{1}(\bar{k})} \bar{\zeta}_{0}^{\prime}
$$

Proof. Replacing $\kappa_{0}$ by $\kappa_{0}^{\prime}-\frac{i}{3} B_{0} \rho_{0}$ from (6.8), and $\zeta_{0}$ by $\frac{\zeta_{0}^{\prime}}{\overline{\mathcal{L}}_{1}(k)}$ from (5.4), we indeed obtain

$$
\begin{aligned}
d G= & \mathcal{T}(G) \rho_{0}+\mathcal{L}_{1}(G) \kappa_{0}+\mathcal{K}(G) \zeta_{0}+\overline{\mathcal{L}}_{1}(G) \bar{\kappa}_{0}+\overline{\mathcal{K}}(G) \bar{\zeta}_{0} \\
= & \mathcal{T}(G) \rho_{0}+\mathcal{L}_{1}(G)\left(\kappa_{0}^{\prime}-\frac{i}{3} B_{0} \rho_{0}\right)+\mathcal{K}(G) \frac{\zeta_{0}^{\prime}}{\overline{\mathcal{L}}_{1}(k)} \\
& +\overline{\mathcal{L}}_{1}(G)\left(\bar{\kappa}_{0}^{\prime}+\frac{i}{3} \bar{B}_{0} \rho_{0}\right)+\overline{\mathcal{K}}(G) \frac{\bar{\zeta}_{0}^{\prime}}{\mathcal{L}_{1}(\bar{k})} .
\end{aligned}
$$


Using this lemma for $d K_{0}^{2 \prime}, d K_{0}^{3 \prime}$, and replacing also $d \rho_{0}, d \kappa_{0}^{\prime}, d \zeta_{0}^{\prime}, d \bar{\kappa}_{0}^{\prime}, d \bar{\zeta}_{0}^{\prime}$ by means of (7.1), we have

$$
\begin{aligned}
& 0=0 \quad+0 \quad-K_{0}^{1 \prime} \rho_{0} \wedge \zeta_{0}^{\prime} \wedge \bar{\kappa}_{0}^{\prime} \wedge \kappa_{0}^{\prime} \wedge \bar{\zeta}_{0}^{\prime} \\
& +\overline{\mathcal{L}}_{1}\left(K_{0}^{2 \prime}\right) \bar{\kappa}_{0}^{\prime} \wedge \rho_{0} \wedge \zeta_{0}^{\prime} \wedge \kappa_{0}^{\prime} \wedge \bar{\zeta}_{0}^{\prime}+K_{0}^{2 \prime} \bar{R}_{0}^{1 \prime} \rho_{0} \wedge \bar{\kappa}_{0}^{\prime} \wedge \zeta_{0}^{\prime} \wedge \kappa_{0}^{\prime} \wedge \bar{\zeta}_{0}^{\prime}-K_{0}^{2 \prime} \rho_{0} \wedge Z_{0}^{8 \prime} \zeta_{0}^{\prime} \wedge \bar{\kappa}_{0}^{\prime} \wedge \bar{\zeta}_{0}^{\prime} \\
& +\frac{\mathcal{K}\left(K_{0}^{3 \prime}\right)}{\overline{\mathcal{L}}_{1}(k)} \zeta_{0}^{\prime} \wedge \rho_{0} \wedge \bar{\kappa}_{0}^{\prime} \wedge \kappa_{0}^{\prime} \wedge \bar{\zeta}_{0}^{\prime}+K_{0}^{3 \prime} R_{0}^{2 \prime} \rho_{0} \wedge \zeta_{0}^{\prime} \wedge \bar{\kappa}_{0}^{\prime} \wedge \kappa_{0}^{\prime} \wedge \bar{\zeta}_{0}^{\prime}-0 \\
& +0 \\
& +K_{0}^{5 \prime} K_{0}^{3 \prime} \rho_{0} \wedge \bar{\kappa}_{0}^{\prime} \wedge \zeta_{0}^{\prime} \wedge \kappa_{0}^{\prime} \wedge \bar{\zeta}_{0}^{\prime}-0 \\
& +0 \\
& +K_{0}^{6 \prime} K_{0}^{2 \prime} \rho_{0} \wedge \zeta_{0}^{\prime} \wedge \bar{\kappa}_{0}^{\prime} \wedge \kappa_{0}^{\prime} \wedge \bar{\zeta}_{0}^{\prime}-0 \\
& +0 \\
& +Z_{0}^{2 \prime} \rho_{0} \wedge \zeta_{0}^{\prime} \wedge \bar{\kappa}_{0}^{\prime} \wedge \kappa_{0}^{\prime} \wedge \bar{\zeta}_{0}^{\prime} \quad-\zeta_{0}^{\prime} \wedge \bar{K}_{0}^{1 \prime} \wedge \rho_{0} \wedge \bar{\kappa}_{0}^{\prime} \wedge \kappa_{0}^{\prime} \wedge \bar{\zeta}_{0}^{\prime},
\end{aligned}
$$

hence caring about signs when factoring by the naturally appearing 5 -form

$$
\begin{aligned}
& 0=\rho_{0} \wedge \kappa_{0}^{\prime} \wedge \zeta_{0}^{\prime} \wedge \bar{\kappa}_{0}^{\prime} \wedge \bar{\zeta}_{0}^{\prime}\left(0 \quad+0 \quad-K_{0}^{1 \prime}\right. \\
& \overline{\mathcal{L}}_{1}\left(K_{0}^{2 \prime}\right)-K_{0}^{2 \prime} \bar{R}_{0}^{1 \prime}-K_{0}^{2 \prime} Z_{0}^{8 \prime} \\
& -\frac{\mathcal{K}\left(K_{0}^{3 \prime}\right)}{\overline{\mathcal{L}}_{1}(k)}+K_{0}^{3 \prime} R_{0}^{2 \prime}-0 \\
& +0 \quad-K_{0}^{5 \prime} K_{0}^{3 \prime}-0 \\
& +0 \quad+K_{0}^{6 \prime} K_{0}^{2 \prime}-0 \\
& \left.+0+Z_{0}^{2 \prime}+\bar{K}_{0}^{1 \prime}\right),
\end{aligned}
$$

whence we arrive at the announced first identity (12.4) by remembering some useful relations

$$
\frac{\mathcal{K}\left(K_{0}^{3 \prime}\right)}{\overline{\mathcal{L}}_{1}(k)}=\overline{\mathcal{L}}_{1}\left(K_{0}^{2 \prime}\right)+K_{0}^{2 \prime} K_{0}^{6 \prime}-K_{0}^{2 \prime}(\underbrace{R_{0}^{1 \prime}+Z_{0}^{8 \prime}}_{=2 K_{0}^{6 \prime}})+K_{0}^{3 \prime}(\underbrace{R_{0}^{2 \prime}-K_{0}^{5 \prime}}_{=0 !})-K_{0}^{1 \prime}+\bar{K}_{0}^{1 \prime}+Z_{0}^{2 \prime} .
$$

For the second identity (12.5), we proceed similarly, applying the exterior differentiation operator $d$ to the structure equation for $d \zeta_{0}^{\prime}$ from (7.1)

$$
\begin{aligned}
0 & =d^{2} \zeta_{0}^{\prime} \\
& =\underbrace{d\left(\boldsymbol{Z}_{0}^{2 \prime}\right) \wedge \rho_{0} \wedge \zeta_{0}^{\prime}}_{\text {don't want }}+Z_{0}^{2 \prime} d \rho_{0} \wedge \zeta_{0}^{\prime}-Z_{0}^{2 \prime} \rho_{0} \wedge d \zeta_{0}^{\prime} \\
& +\underbrace{d\left(\boldsymbol{Z}_{0}^{5 \prime}\right) \wedge \kappa_{0}^{\prime} \wedge \zeta_{0}^{\prime}}_{\text {want }}+Z_{0}^{5 \prime} d \kappa_{0}^{\prime} \wedge \zeta_{0}^{\prime}-Z_{0}^{5 \prime} \kappa_{0}^{\prime} \wedge d \zeta_{0}^{\prime} \\
& +\underbrace{d\left(\boldsymbol{Z}_{0}^{8 \prime}\right) \wedge \zeta_{0}^{\prime} \wedge \bar{\kappa}_{0}^{\prime}}_{\text {want }}+Z_{0}^{8 \prime} d \zeta_{0}^{\prime} \wedge \bar{\kappa}_{0}^{\prime}-Z_{0}^{8 \prime} \zeta_{0}^{\prime} \wedge d \bar{\kappa}_{0}^{\prime} \\
& +\underbrace{d\left(\boldsymbol{Z}_{0}^{9 \prime}\right) \wedge \zeta_{0}^{\prime} \wedge \bar{\zeta}_{0}^{\prime}}_{\text {don't want }}+Z_{0}^{9 \prime} d \zeta_{0}^{\prime} \wedge \bar{\zeta}_{0}^{\prime}-Z_{0}^{9 \prime} \zeta_{0}^{\prime} \wedge d \bar{\zeta}_{0}^{\prime} .
\end{aligned}
$$


Observe that the desired identity involves the derivatives of $Z_{0}^{5 \prime}$ and $Z_{0}^{8 \prime}$. Hence we may conserve those terms marked 'want' by wedging with the appropriate 2-form $\rho_{0} \wedge \bar{\zeta}_{0}^{\prime}$

$$
\begin{aligned}
& 0=0 \\
& +Z_{0}^{2 \prime} d \rho_{0} \wedge \zeta_{0}^{\prime} \wedge \rho_{0} \wedge \bar{\zeta}_{0}^{\prime}+0 \\
& +d Z_{0}^{5 \prime} \wedge \kappa_{0}^{\prime} \wedge \zeta_{0}^{\prime} \wedge \rho_{0} \wedge \bar{\zeta}_{0}^{\prime}+Z_{0}^{5 \prime} d \kappa_{0}^{\prime} \wedge \zeta_{0}^{\prime} \wedge \rho_{0} \wedge \bar{\zeta}_{0}^{\prime}-Z_{0}^{5 \prime} \kappa_{0}^{\prime} \wedge d \zeta_{0}^{\prime} \wedge \rho_{0} \wedge \bar{\zeta}_{0}^{\prime} \\
& +d Z_{0}^{8 \prime} \wedge \zeta_{0}^{\prime} \wedge \bar{\kappa}_{0}^{\prime} \wedge \rho_{0} \wedge \bar{\zeta}_{0}^{\prime}+Z_{0}^{8 \prime} d \zeta_{0}^{\prime} \wedge \bar{\kappa}_{0}^{\prime} \wedge \rho_{0} \wedge \bar{\zeta}_{0}^{\prime}-Z_{0}^{8 \prime} \zeta_{0}^{\prime} \wedge d \bar{\kappa}_{0}^{\prime} \wedge \rho_{0} \wedge \bar{\zeta}_{0}^{\prime} \\
& +0 \\
& +0
\end{aligned}
$$

Using Lemma 12.6 for $d \boldsymbol{Z}_{0}^{5 \prime}, d \boldsymbol{Z}_{0}^{8 \prime}$, and replacing also $d \rho_{0}, d \kappa_{0}^{\prime}, d \zeta_{0}^{\prime}, d \bar{\kappa}_{0}^{\prime}, d \bar{\zeta}_{0}^{\prime}$ by means of (7.1), we have

$$
\begin{aligned}
0= & 0 \quad+Z_{0}^{2 \prime} i \kappa_{0}^{\prime} \wedge \bar{\kappa}_{0}^{\prime} \wedge \zeta_{0}^{\prime} \wedge \rho_{0} \wedge \bar{\zeta}_{0}^{\prime}+0 \\
& +\overline{\mathcal{L}}_{1}\left(Z_{0}^{5 \prime}\right) \bar{\kappa}_{0}^{\prime} \wedge \kappa_{0}^{\prime} \wedge \zeta_{0}^{\prime} \wedge \rho_{0} \wedge \bar{\zeta}_{0}^{\prime}+Z_{0}^{5 \prime} K_{0}^{6 \prime} \kappa_{0}^{\prime} \wedge \bar{\kappa}_{0}^{\prime} \wedge \zeta_{0}^{\prime} \wedge \rho_{0} \wedge \bar{\zeta}_{0}^{\prime}+Z_{0}^{5 \prime} \kappa_{0}^{\prime} \wedge Z_{0}^{8 \prime} \zeta_{0}^{\prime} \wedge \bar{\kappa}_{0}^{\prime} \wedge \rho_{0} \wedge \bar{\zeta}_{0}^{\prime} \\
& +\mathcal{L}_{1}\left(Z_{0}^{8 \prime}\right) \kappa_{0}^{\prime} \wedge \zeta_{0}^{\prime} \wedge \bar{\kappa}_{0}^{\prime} \wedge \rho_{0} \wedge \bar{\zeta}_{0}^{\prime}+Z_{0}^{8 \prime} Z_{0}^{5 \prime} \kappa_{0}^{\prime} \wedge \zeta_{0}^{\prime} \wedge \bar{\kappa}_{0}^{\prime} \wedge \rho_{0} \wedge \bar{\zeta}_{0}^{\prime}+Z_{0}^{8 \prime} \zeta_{0}^{\prime} \wedge \bar{K}_{0}^{6 \prime} \wedge \bar{\kappa}_{0}^{\prime} \wedge \kappa_{0}^{\prime} \wedge \rho_{0} \wedge \bar{\zeta}_{0}^{\prime} \\
& +0 \\
& +0
\end{aligned}
$$

hence caring about signs when factoring by the naturally appearing 5 -form, we arrive at the announced second identity (12.5)

$$
\begin{aligned}
& 0=\rho_{0} \wedge \kappa_{0}^{\prime} \wedge \zeta_{0}^{\prime} \wedge \bar{\kappa}_{0}^{\prime} \wedge \bar{\zeta}_{0}^{\prime}\left(0 \quad+i Z_{0}^{2 \prime}+0\right. \\
& -\overline{\mathcal{L}}_{1}\left(Z_{0}^{5 \prime}\right)+Z_{0}^{5 \prime} K_{0}^{6 \prime}+\underline{Z}_{0}^{5 \prime} Z_{0}^{8 \prime} 。 \\
& -\mathcal{L}_{1}\left(Z_{0}^{8 \prime}\right)-\underline{Z_{0}^{8 \prime} Z_{0}^{5 \prime}}+Z_{0}^{8 \prime} \bar{K}_{0}^{6 \prime} \\
& +0 \quad+0 \quad-0 \quad) \text {. }
\end{aligned}
$$

\section{SUMMARIZED STRUCTURE EQUATIONS}

All this work conducted us to finalize the statement of Proposition 10.3, but before, let us make an ample summary.

After normalizations of the group parameters $f, b, d$, the equivalence problem for 2-nondegenerate (constant) Levi rank $1 \mathcal{C}^{\omega}$ or $\mathcal{C}^{\infty}$ real hypersurfaces $M^{5} \subset \mathbb{C}^{3}$ conducts to a 4-dimensional $G$ structure

$$
\left(\begin{array}{ccc}
\mathrm{c} \overline{\mathrm{c}} & 0 & 0 \\
-i \overline{\mathrm{ce}} & \mathrm{c} & 0 \\
-\frac{i}{2} \frac{\overline{\mathrm{cee}}}{\mathrm{c}} & \mathrm{e} & \frac{\mathrm{c}}{\overline{\mathrm{c}}}
\end{array}\right),
$$

where $c \in \mathbb{C}^{*}$ and $e \in \mathbb{C}$, with Maurer-Cartan forms (conjutates are not written)

$$
\begin{aligned}
\alpha & :=\frac{d \mathrm{c}}{\mathrm{c}}, \\
\beta & :=i \frac{\mathrm{e} d \mathrm{c}}{\mathrm{cc}}-i \frac{\mathrm{e} d \overline{\mathrm{c}}}{\mathrm{c} \overline{\mathrm{c}}}-i \frac{d \mathrm{e}}{\mathrm{c}} .
\end{aligned}
$$

Furthermore, 2 fundamental primary differential invariants occur

$$
J=\frac{i}{\overline{\mathrm{CCC}}} \overline{\boldsymbol{J}}_{0} \quad \text { and } \quad W=\frac{1}{\mathrm{c}} W_{0},
$$


where $J_{0}$ and $W_{0}$ are explicit functions on $M$, together with 1 secondary invariant

$$
\begin{aligned}
R & :=\operatorname{Re} Z^{2} \\
& =\operatorname{Re}\left[i \frac{\mathrm{e}}{\mathrm{cc}} W_{0}+\frac{1}{\mathrm{cc}}\left(-\frac{i}{2} \overline{\mathcal{L}}_{1}\left(W_{0}\right)+\frac{i}{2}\left(-\frac{1}{3} \frac{\overline{\mathcal{L}}_{1}\left(\overline{\mathcal{L}}_{1}(k)\right)}{\overline{\mathcal{L}}_{1}(k)}+\frac{1}{3} \bar{P}\right) W_{0}\right)\right] .
\end{aligned}
$$

On the 10-dimensional manifold $M^{5} \times G^{4} \times \mathbb{R}$ equipped with coordinates

$$
\left(z_{1}, z_{2}, \bar{z}_{1}, \bar{z}_{2}, v\right) \times(\mathrm{c}, \overline{\mathrm{c}}, \mathrm{e}, \overline{\mathrm{e}}) \times(\mathrm{t}),
$$

there are two modified-prolonged Maurer-Cartan forms

$$
\begin{aligned}
& \pi^{1}:=\alpha-\left(\mathrm{t}-\frac{i}{2} \operatorname{Im} Z^{2}\right) \rho-\left(R^{1}-\bar{K}^{6}\right) \kappa-R^{2} \zeta-K^{6} \bar{\kappa}-0, \\
& \pi^{2}:=\beta-i Z^{1} \rho-\left(\mathrm{t}-\frac{i}{2} \operatorname{Im} Z^{2}+K^{1}\right) \kappa-K^{2} \zeta-K^{3} \bar{\kappa}-K^{4} \bar{\zeta}
\end{aligned}
$$

where $R^{i}, K^{i}, Z^{i}$ are explicit functions on $M^{5} \times G^{4}$.

Theorem 13.1. After finalization of absorption, the structure equations read

$$
\begin{aligned}
d \rho= & \left(\pi^{1}+\bar{\pi}^{1}\right) \wedge \rho+i \kappa \wedge \bar{\kappa}, \\
d \kappa= & \pi^{2} \wedge \rho+\pi^{1} \wedge \kappa+\zeta \wedge \bar{\kappa}, \\
d \zeta= & \left(\pi^{1}-\bar{\pi}^{1}\right) \wedge \zeta+i \pi^{2} \wedge \kappa \\
& +R \rho \wedge \zeta+J \rho \wedge \bar{\kappa}+W \kappa \wedge \zeta .
\end{aligned}
$$

14. THE FINAL $\{e\}$-STRUCTURE

Let $\Omega_{1}$ and $\Omega_{2}$ be the two 2-forms defined by:

$$
\begin{aligned}
& \Omega_{1}:=d \pi^{1}-i \kappa \wedge \bar{\pi}^{2}-\zeta \wedge \bar{\zeta}, \\
& \Omega_{2}:=d \pi^{2}-\pi^{2} \wedge \bar{\pi}^{1}-\zeta \wedge \bar{\pi}^{2} .
\end{aligned}
$$

When the two fundamental invariants $J_{0} \equiv 0 \equiv W_{0}$ vanish identically, since we know that

$$
\begin{aligned}
R & =\operatorname{Re}\left[i \frac{\mathrm{e}}{\mathrm{cc}} W_{0}+\frac{1}{\mathrm{c} \overline{\mathrm{C}}}\left(-\frac{i}{2} \overline{\mathcal{L}}_{1}\left(W_{0}\right)+\frac{i}{2}\left(-\frac{1}{3} \frac{\overline{\mathcal{L}}_{1}\left(\overline{\mathcal{L}}_{1}(k)\right)}{\overline{\mathcal{L}}_{1}(k)}+\frac{1}{3} \bar{P}\right) W_{0}\right)\right], \\
J & =\frac{i}{\overline{\mathrm{CCC}}} \overline{\boldsymbol{J}}_{0}, \\
W & =\frac{1}{\mathrm{c}} W_{0},
\end{aligned}
$$

it comes

$$
0 \equiv R \equiv J \equiv W .
$$

Independently, the addendum to [18] shows that in the case where all invariants vanish, these auxiliary 2 -forms $\Omega_{1}$ and $\Omega_{2}$ satisfy

$$
\begin{aligned}
\left(\Omega_{1}+\bar{\Omega}_{1}\right) \wedge \rho & =0, \\
\Omega_{2} \wedge \rho+\Omega_{1} \wedge \kappa & =0, \\
\left(\Omega_{1}-\bar{\Omega}_{1}\right) \wedge \zeta+i \Omega_{2} \wedge \kappa & =0 .
\end{aligned}
$$

In general, the right-hand sides of these structure equations are not necessarily zero, and they depend on the invariants $R, J, W$. 
Proposition 14.1. The two 2-forms $\Omega_{1}$ and $\Omega_{2}$ satisfy

$$
\begin{aligned}
\left(\Omega_{1}+\bar{\Omega}_{1}\right) \wedge \rho= & 0, \\
\Omega_{2} \wedge \rho+\Omega_{1} \wedge \kappa= & -R \rho \wedge \zeta \wedge \bar{\kappa}-W \kappa \wedge \zeta \wedge \bar{\kappa}, \\
i \Omega_{2} \wedge \kappa+\left(\Omega_{1}-\bar{\Omega}_{1}\right) \wedge \zeta= & -d R \wedge \rho \wedge \zeta-R\left(\pi^{1}+\bar{\pi}^{1}\right) \wedge \rho \wedge \zeta-i R \pi^{2} \wedge \rho \wedge \kappa \\
& +i R \kappa \wedge \zeta \wedge \bar{\zeta}-d J \wedge \rho \wedge \bar{\kappa}-3 J \bar{\pi}^{1} \wedge \rho \wedge \bar{\kappa} \\
& -J \rho \wedge \kappa \wedge \bar{\zeta}-d W \wedge \kappa \wedge \zeta-W \pi^{2} \wedge \rho \wedge \zeta \\
& -W \pi^{1} \wedge \kappa \wedge \zeta-W J \rho \wedge \kappa \wedge \bar{\kappa} .
\end{aligned}
$$

Proof. These relations come from Poincaré's identities

$$
0 \equiv d \circ d \rho \equiv d \circ d \kappa \equiv d \circ d \zeta
$$

applied to the finalized structure equations of Theorem 13.1, in which $d \rho, d \kappa$, $d \zeta$ should be replaced again using Theorem 13.1, followed by a reorganization of the obtained 3-forms.

For the first line (14.2)

$$
\begin{aligned}
0= & d \circ d \rho \\
= & \left(d \pi^{1}+d \bar{\pi}^{1}\right) \wedge \rho-\left(\pi^{1}+\bar{\pi}^{1}\right) \wedge d \rho+i d \kappa \wedge \bar{\kappa}-i \kappa \wedge d \bar{\kappa} \\
= & \left(d \pi^{1}+d \bar{\pi}^{1}\right) \wedge \rho-\left(\pi^{1}+\bar{\pi}^{1}\right) \wedge\left(\left(\underline{\pi^{1}+\bar{\pi}^{1}}\right) \wedge \rho+i \kappa \wedge \bar{\kappa}\right) \\
& +i\left(\pi^{2} \wedge \rho+\pi^{1} \wedge \rho+\underline{\zeta \wedge \bar{\kappa}_{\circ}}\right) \wedge \bar{\kappa}-i \kappa \wedge\left(\bar{\pi}^{2} \wedge \rho+\bar{\pi}^{1} \wedge \bar{\kappa}+\underline{\bar{\zeta} \wedge \kappa_{\circ}}\right) .
\end{aligned}
$$

Afer simplification, this becomes

$$
0=\left(d \pi^{1}-i \kappa \wedge \bar{\pi}^{2}\right) \wedge \rho+\left(d \bar{\pi}^{1}+i \bar{\kappa} \wedge \pi^{2}\right) \wedge \rho,
$$

and after insertion of twice $-\zeta \wedge \bar{\zeta}$ which is purely imaginary - hence disappears - , we obtain (14.2)

$$
\begin{aligned}
0 & =\left(d \pi^{1}-i \kappa \wedge \bar{\pi}^{2}-\zeta \wedge \bar{\zeta}\right) \wedge \rho+\left(d \bar{\pi}^{1}+i \bar{\kappa} \wedge \pi^{2}-\bar{\zeta} \wedge \zeta\right) \wedge \rho \\
& =\Omega_{1} \wedge \rho+\bar{\Omega}_{1} \wedge \rho .
\end{aligned}
$$

For (14.3), we proceed analogously, starting from the second structure equation of Theorem 13.1

$$
\begin{aligned}
0= & d \circ d \kappa \\
= & d \pi^{2} \wedge \rho-\pi^{2} \wedge d \rho+d \pi^{1} \wedge \kappa-\pi^{1} \wedge d \kappa+d \zeta \wedge \bar{\kappa}-\zeta \wedge d \bar{\kappa} \\
= & d \pi^{2} \wedge \rho-\pi^{2} \wedge\left(\left(\pi^{1}+\bar{\pi}^{1}\right) \wedge \rho+i \kappa \wedge \bar{\kappa}\right)+d \pi^{1} \wedge \kappa-\pi^{1} \wedge\left(\pi^{2} \wedge \rho+\zeta \wedge \bar{\kappa}\right) \\
& +\left(\left(\pi^{1}-\bar{\pi}^{1}\right) \wedge \zeta+i \pi^{2} \wedge \kappa+R \rho \wedge \zeta+W \kappa \wedge \zeta\right) \wedge \bar{\kappa}-\zeta \wedge\left(\bar{\pi}^{2} \wedge \rho+\bar{\pi}^{1} \wedge \bar{\kappa}+\bar{\zeta} \wedge \kappa\right) .
\end{aligned}
$$

After four annihilations by pairs and a reorganization, this becomes

$$
\begin{aligned}
0= & d \pi^{2} \wedge \rho-\bar{\pi}^{2} \wedge \pi^{1} \wedge \rho_{1}-\pi^{2} \wedge \bar{\pi}^{1} \wedge \rho-\underline{i \pi^{2} \wedge \kappa \wedge \bar{\kappa}_{2}}+d \pi^{1} \wedge \kappa-\underline{\pi^{1} \wedge \pi^{2} \wedge \rho_{1}-\pi^{1} \wedge \zeta \wedge \bar{\kappa}_{3}} \\
& +\frac{\pi^{1} \wedge \zeta \wedge \bar{\kappa}_{3}}{-\bar{\pi}^{1} \wedge \zeta \wedge \bar{\kappa}_{4}}+\underline{i \pi^{2} \wedge \kappa \wedge \bar{\kappa}_{2}}+R \rho \wedge \zeta \wedge \bar{\kappa}+W \kappa \wedge \zeta \wedge \bar{\kappa}-\zeta \wedge \bar{\pi}^{2} \wedge \rho \\
& -\underline{\zeta \wedge \bar{\pi}^{1} \wedge \bar{\kappa}_{4}}-\zeta \wedge \bar{\zeta} \wedge \kappa \\
= & \left(d \pi^{2}-\pi^{2} \wedge \bar{\pi}^{1}-\zeta \wedge \bar{\pi}^{2}\right) \wedge \rho+\left(d \pi^{1}-\zeta \wedge \bar{\zeta}\right) \wedge \kappa \\
& +R \rho \wedge \zeta \wedge \bar{\kappa}+W \kappa \wedge \zeta \wedge \bar{\kappa},
\end{aligned}
$$


which is (14.3), since we can insert $\left(-i \kappa \wedge \bar{\pi}^{2}\right) \wedge \kappa=0$. Lastly

$$
\begin{aligned}
0= & d \circ d \zeta \\
= & i d \pi^{2} \wedge \kappa-i \pi^{2} \wedge d \kappa+d \pi^{1} \wedge \zeta-\pi^{1} \wedge d \zeta-d \bar{\pi}^{1} \wedge \zeta+\bar{\pi}^{1} \wedge d \zeta \\
& +d R \wedge \rho \wedge \zeta+R d \rho \wedge \zeta-R \rho \wedge d \zeta \\
& +d J \wedge \rho \wedge \bar{\kappa}+J d \rho \wedge \bar{\kappa}-J \rho \wedge d \bar{\kappa} \\
& +d W \wedge \kappa \wedge \zeta+W d \kappa \wedge \zeta-W \kappa \wedge d \zeta,
\end{aligned}
$$

whence by replacements

$$
\begin{aligned}
0= & i d \pi^{2} \wedge \kappa-i \pi^{2} \wedge\left(\pi^{1} \wedge \kappa+\zeta \wedge \bar{\kappa}\right)+d \pi^{1} \wedge \zeta-\pi^{1} \wedge\left(i \pi^{2} \wedge \kappa-\bar{\pi}^{1} \wedge \zeta+R \rho \wedge \zeta\right. \\
& +J \rho \wedge \bar{\kappa}+W \kappa \wedge \zeta)-d \bar{\pi}^{1} \wedge \zeta+\bar{\pi}^{1} \wedge\left(i \pi^{2} \wedge \kappa+\pi^{1} \wedge \zeta+R \rho \wedge \zeta+J \rho \wedge \bar{\kappa}+W \kappa \wedge \zeta\right) \\
& +d R \wedge \rho \wedge \zeta+R\left(\left(\pi^{1}+\bar{\pi}^{1}\right) \wedge \rho+i \kappa \wedge \bar{\kappa}\right) \wedge \zeta-R \rho \wedge\left(i \pi^{2} \wedge \kappa+\left(\pi^{1}-\bar{\pi}^{1}\right) \wedge \zeta+W \kappa \wedge \zeta\right) \\
& +d J \wedge \rho \wedge \bar{\kappa}+J\left(\pi^{1}+\bar{\pi}^{1}\right) \wedge \rho \wedge \bar{\kappa}-J \rho \wedge\left(\bar{\pi}^{1} \wedge \bar{\kappa}+\bar{\zeta} \wedge \kappa\right) \\
& +d W \wedge \kappa \wedge \zeta+W\left(\pi^{2} \wedge \rho+\pi^{1} \wedge \kappa\right) \wedge \zeta-W \kappa \wedge\left(\left(\pi^{1}-\bar{\pi}^{1}\right) \wedge \zeta+R \rho \wedge \zeta+J \rho \wedge \bar{\kappa}\right) .
\end{aligned}
$$

Let us expand this and underline the eight annihilating pairs

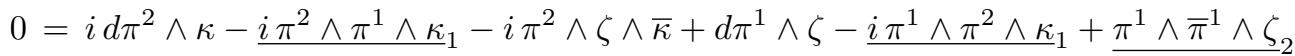

$$
\begin{aligned}
& -\underline{R \pi^{1} \wedge \rho \wedge \zeta_{3}}-\underline{J \pi^{1} \wedge \rho \wedge \bar{\kappa}_{6}}-\underline{W \pi^{1} \wedge \kappa \wedge \zeta_{7}}-d \bar{\pi}^{1} \wedge \zeta+i \bar{\pi}^{1} \wedge \pi^{2} \wedge \kappa+\bar{\pi}^{1} \wedge \pi^{1} \wedge \zeta_{2} \\
& +\underline{R \bar{\pi}^{1} \wedge \rho \wedge \zeta_{4}}+J \bar{\pi}^{1} \wedge \rho \wedge \bar{\kappa}+\underline{W \bar{\pi}^{1} \wedge \kappa \wedge \zeta_{8}}+d R \wedge \rho \wedge \zeta+\underline{R \pi^{1} \wedge \rho \wedge \zeta_{3}+R \bar{\pi}^{1} \wedge \rho \wedge \zeta}
\end{aligned}
$$

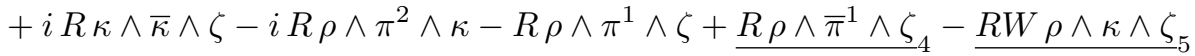

$$
\begin{aligned}
& +d J \wedge \rho \wedge \bar{\kappa}+J \pi^{1} \wedge \rho \wedge \bar{\kappa}_{6}+J \bar{\pi}^{1} \wedge \rho \wedge \bar{\kappa}-J \rho \wedge \bar{\pi}^{1} \wedge \bar{\kappa}-J \rho \wedge \bar{\zeta} \wedge \kappa+d W \wedge \kappa \wedge \zeta
\end{aligned}
$$

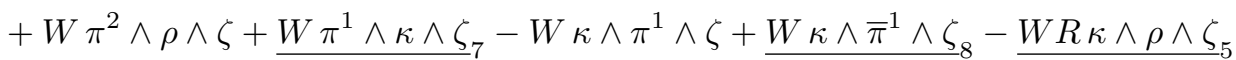

$$
\begin{aligned}
& -W J \kappa \wedge \rho \wedge \bar{\kappa} \text {. }
\end{aligned}
$$

After simplification and reorganization

$$
\begin{aligned}
0= & i\left(d \pi^{2}-\pi^{2} \wedge \bar{\pi}^{1}\right) \wedge \kappa+\left(d \pi^{1}-d \bar{\pi}^{1}-i \bar{\kappa} \wedge \pi^{2}\right) \wedge \zeta \\
& +d R \wedge \rho \wedge \zeta+R \bar{\pi}^{1} \wedge \rho \wedge \zeta-i R \kappa \wedge \zeta \wedge \bar{\kappa}+i R \pi^{2} \wedge \rho \wedge \kappa+R \pi^{1} \wedge \rho \wedge \zeta \\
& +d J \wedge \rho \wedge \bar{\kappa}+3 J \bar{\pi}^{1} \wedge \rho \wedge \bar{\kappa}+J \rho \wedge \kappa \wedge \bar{\zeta} \\
& +d W \wedge \kappa \wedge \zeta+W \pi^{2} \wedge \rho \wedge \zeta+W \pi^{1} \wedge \kappa \wedge \zeta+W J \rho \wedge \kappa \wedge \bar{\kappa} .
\end{aligned}
$$

To reach (14.4) completely, only the first line must yet be transformed, and it suffices to insert into it two terms which cancel together

$$
i\left(d \pi^{2}-\pi^{2} \wedge \bar{\pi}^{1}-\underline{\zeta \wedge \bar{\pi}^{2}}{ }_{0}\right) \wedge \kappa+\left(d \pi^{1}-\underline{i \kappa \wedge \bar{\pi}^{2}}{ }_{\circ}-d \bar{\pi}^{1}-i \bar{\kappa} \wedge \pi^{2}\right) \wedge \zeta .
$$

Remind that all present considerations hold on the 9-dimensional manifold $M^{5} \times G^{4}$ equipped with the coordinates

$$
\left(z_{1}, z_{2}, \bar{z}_{1}, \bar{z}_{2}, v\right) \times(\mathrm{c}, \mathrm{e}, \overline{\mathrm{c}}, \overline{\mathrm{e}}),
$$

the supplementary real variable $t \in \mathbb{R}$ being considered as a parameter until it becomes a variable at the very end of the process for an $\{e\}$-structure on the 10-dimensional manifold 
$M^{5} \times G^{4} \times \mathbb{R}$. In order to build up such an $\{e\}$-structure, the goal now is to fully determine the two 2 -forms $\Omega_{1}, \Omega_{2}$, and precisely, to determine how they express in terms of the coframe

$$
\left\{\pi^{1}, \pi^{2}, \bar{\pi}^{1}, \bar{\pi}^{2}, \rho, \kappa, \zeta, \bar{\kappa}, \bar{\zeta}\right\} .
$$

To begin with, suppose that there are two ways of solving for $\left\{\Omega_{1}, \Omega_{2}\right\}$ the structure equations of Proposition 14.1, leading to another set of solutions $\left\{\Omega_{1}^{\prime}, \Omega_{2}^{\prime}\right\}$. Then their differences $\Gamma_{1}:=$ $\Omega_{1}^{\prime}-\Omega_{1}$ and $\Gamma_{2}:=\Omega_{2}^{\prime}-\Omega_{2}$ must necessarily satisfy the homogeneous equations

$$
\begin{aligned}
\left(\Gamma_{1}+\bar{\Gamma}_{1}\right) \wedge \rho=0, \\
\Gamma_{2} \wedge \rho+\Gamma_{1} \wedge \kappa=0, \\
i \Gamma_{2} \wedge \kappa+\left(\Gamma_{1}-\bar{\Gamma}_{1}\right) \wedge \zeta=0 .
\end{aligned}
$$

The addendum to the article [18] provides a detailed proof of the elementary

Proposition 14.5. The general solution $\left\{\Gamma_{1}, \Gamma_{2}\right\}$ to these homogeneous equations is given by

$$
\Gamma_{1}:=\Lambda \wedge \rho, \quad \Gamma_{2}:=\Lambda \wedge \kappa+h \rho \wedge \kappa,
$$

where $\Lambda$ is a real 1 -form and $h$ is purely imaginary function.

This means that the two sets of solutions are related to each other by

$$
\Omega_{1}^{\prime}=\Omega_{1}+\Lambda \wedge \rho, \quad \Omega_{2}^{\prime}=\Omega_{2}+\Lambda \wedge \kappa+h \rho \wedge \kappa .
$$

Due to this flexibility represented by $\Lambda, h$, it will be necessary to prolong the structure equations by adding this real 1 -form:

$$
\Lambda=d \mathrm{t}+\cdots,
$$

the remainder terms being very complicated, while the function $h$ could be some new invariant. However, it will be later shown that $h$ expresses in terms of the $3^{\text {rd }}$-order jets of $W$ and $J$, thus eliminating the possibility of appearance of new primary CR invariants. On the other hand, the existence of $\Lambda$ can be explained by an application (not detailed here) of Cartan's test, due to the fact that there is one degree of real-valued indeterminancy during the fourth absorption.

It therefore suffices to find a particular set of solution $\Omega_{1}$ and $\Omega_{2}$, and then to parametrize the solution space by means of $\Lambda, h$. We will adopt the following strategy. First, we will find the simplest forms for $\Omega_{1}$ and $\Omega_{2}$ restrained by the first two equations (14.2), (14.3) of the starting Proposition 14.1. Then we will simplify these 2 -forms by means of Cartan's lemma to eliminate as many unknown variables as possible using the third, more subtle, equation (14.4). At the end of the elimination, those remaining unknowns which cannot be computed due to the lack of information turn out to behave like $\Lambda$ and $h$, and hence we will terminate the process of solving for solutions.

In $M^{5} \times G^{4}$, it will be useful to adopt the following notations for the covariant derivatives

$$
\begin{aligned}
d R & =R_{\pi^{1}} \pi^{1}+R_{\pi^{2}} \pi^{2}+R_{\bar{\pi}^{1}} \bar{\pi}^{1}+R_{\bar{\pi}^{2}} \bar{\pi}^{2}+R_{\rho} \rho+R_{\kappa} \kappa+R_{\zeta} \zeta+R_{\bar{\kappa}} \bar{\kappa}+R_{\bar{\zeta}} \bar{\zeta}, \\
d J & =J_{\pi^{1}} \pi^{1}+J_{\pi^{2}} \pi^{2}+J_{\bar{\pi}^{1}} \bar{\pi}^{1}+J_{\bar{\pi}^{2}} \bar{\pi}^{2}+J_{\rho} \rho+J_{\kappa} \kappa+J_{\zeta} \zeta+J_{\bar{\kappa}} \bar{\kappa}+J_{\bar{\zeta}} \bar{\zeta}, \\
d W & =W_{\pi^{1}} \pi^{1}+W_{\pi^{2}} \pi^{2}+W_{\bar{\pi}^{1}} \bar{\pi}^{1}+W_{\bar{\pi}^{2}} \bar{\pi}^{2}+W_{\rho} \rho+W_{\kappa} \kappa+W_{\zeta} \zeta+W_{\bar{\kappa}} \bar{\kappa}+W_{\bar{\zeta}} \bar{\zeta} .
\end{aligned}
$$

Some of these coefficients will be revealed during the course of solving the structure equations. We first turn ourselves to finding the simplest form of $\Omega_{1}, \Omega_{2}$ satisfying only the first two equations (14.2), (14.3).

Proposition 14.7. There exists a real-valued function $p$ and two differential 1-forms $\Pi, \Psi$ such that

$$
\begin{aligned}
& \Omega_{1}=\Pi \wedge \rho+p \kappa \wedge \bar{\kappa}-\bar{W} \kappa \wedge \bar{\zeta}-W \zeta \wedge \bar{\kappa}, \\
& \Omega_{2}=\Psi \wedge \rho+\Pi \wedge \kappa-R \zeta \wedge \bar{\kappa} .
\end{aligned}
$$


Proof. We can rearrange the terms in (14.3)

$$
0=\left(\Omega_{1}+W \zeta \wedge \bar{\kappa}\right) \wedge \kappa+\left(\Omega_{2}+R \zeta \wedge \bar{\kappa}\right) \wedge \rho,
$$

in order that an application of the Cartan Lemma yield functions $\Delta, \Theta, \Pi^{\prime \prime}, \Psi$ so that

$$
\begin{aligned}
\Omega_{1}+W \zeta \wedge \bar{\kappa} & =\Delta \wedge \kappa+\Theta \wedge \rho, \\
\Omega_{2}+R \zeta \wedge \bar{\kappa} & =\Pi^{\prime \prime} \wedge \kappa+\Psi \wedge \rho,
\end{aligned}
$$

with a double prime on $\Pi^{\prime \prime}$ meaning that we will soon modify it two times.

In fact, substituting these representations back into (14.8), we see that there are constraints on $\Theta$ and $\Pi^{\prime \prime}$

$$
\begin{aligned}
0 & =\left(\underline{\Delta \wedge \kappa_{\circ}}+\Theta \wedge \rho\right) \wedge \kappa+\left(\Pi^{\prime \prime} \wedge \kappa+\underline{\Psi \wedge \rho_{\circ}}\right) \wedge \rho \\
& =\left(\Theta-\Pi^{\prime \prime}\right) \wedge \rho \wedge \kappa .
\end{aligned}
$$

By the Cartan Lemma again, this implies the existence of two functions $a, b$ so that $\Theta$ and $\Pi^{\prime \prime}$ are related to each other by

$$
\Theta=\Pi^{\prime \prime}+a \rho+b \kappa .
$$

Next, putting this into the expression of $\Omega_{1}$, while letting $\Pi^{\prime}:=\Pi^{\prime \prime}+b \kappa$, it follows that

$$
\begin{aligned}
\Omega_{1} & =\Delta \wedge \kappa+\Theta \wedge \rho-W \zeta \wedge \bar{\kappa} \\
& =\Delta \wedge \kappa+\left(\Pi^{\prime \prime}+a \rho_{\circ}+b \kappa\right) \wedge \rho-W \zeta \wedge \bar{\kappa} \\
& =\Delta \wedge \kappa+\Pi^{\prime} \wedge \rho-W \zeta \wedge \bar{\kappa},
\end{aligned}
$$

while $\Omega_{2}$ becomes

$$
\begin{aligned}
\Omega_{2} & =\Pi^{\prime \prime} \wedge \kappa+\Psi \wedge \rho-R \zeta \wedge \bar{\kappa} \\
& =\left(\Pi^{\prime \prime}+b \kappa\right) \wedge \kappa+\Psi \wedge \rho-R \zeta \wedge \bar{\kappa} \\
& =\Pi^{\prime} \wedge \kappa+\Psi \wedge \rho-R \zeta \wedge \bar{\kappa} .
\end{aligned}
$$

The next observation is that $\Delta$ can be further simplified. Indeed, let us replace $\Omega_{1}$ in (14.2)

$$
\begin{aligned}
0 & =\left(\Omega_{1}+\bar{\Omega}_{1}\right) \wedge \rho \\
& =\Delta \wedge \kappa \wedge \rho-W \zeta \wedge \bar{\kappa} \wedge \rho+\bar{\Delta} \wedge \bar{\kappa} \wedge \rho-\bar{W} \bar{\zeta} \wedge \kappa \wedge \rho .
\end{aligned}
$$

Then decomposing $\Delta$ as a linear combination along the coframe

$$
\Delta=d_{1} \pi^{1}+d_{2} \pi^{2}+d_{3} \bar{\pi}^{1}+d_{4} \bar{\pi}^{2}+d_{5} \rho+d_{6} \kappa+d_{7} \zeta+d_{8} \bar{\kappa}+d_{9} \bar{\zeta},
$$

we obtain the following values for these coefficients

$$
d_{1}=d_{2}=d_{3}=d_{4}=0, \quad d_{8}=\bar{d}_{8}, \quad d_{9}=\bar{W},
$$

except for $d_{5}$ and $d_{6}$ which on which no constraint is deduced so, and hence

$$
\Delta=d_{5} \rho+d_{6} \kappa+d_{8} \bar{\kappa}+\bar{W} \bar{\zeta} .
$$

Finally, if we write $p:=-d_{8}$ and if we set $\Pi:=\Pi^{\prime}-d_{5} \kappa$, we obtain by reorganization

$$
\begin{aligned}
\Omega_{1} & =\Delta \wedge \kappa+\Pi^{\prime} \wedge \rho-W \zeta \wedge \bar{\kappa} \\
& =\left(d_{5} \rho+\underline{d}_{6} \kappa_{\circ}+d_{8} \bar{\kappa}+\bar{W} \bar{\zeta}\right) \wedge \kappa+\Pi^{\prime} \wedge \rho-W \zeta \wedge \bar{\kappa} \\
& =-d_{8} \kappa \wedge \bar{\kappa}+\left(\Pi^{\prime}-d_{5} \kappa\right) \wedge \rho-\bar{W} \kappa \wedge \bar{\zeta}-W \zeta \wedge \bar{\kappa} \\
& =p \kappa \wedge \bar{\kappa}+\Pi \wedge \rho-\bar{W} \kappa \wedge \bar{\zeta}-W \zeta \wedge \bar{\kappa},
\end{aligned}
$$


and moreover

$$
\begin{aligned}
\Omega_{2} & =\Psi \wedge \rho+\Pi^{\prime} \wedge \kappa-R \zeta \wedge \bar{\kappa} \\
& =\Psi \wedge \rho+\left(\Pi^{\prime}-d_{5} \kappa\right) \wedge \kappa-R \zeta \wedge \bar{\kappa} \\
& =\Psi \wedge \rho+\Pi \wedge \kappa-R \zeta \wedge \bar{\kappa} .
\end{aligned}
$$

Now, using the representations of $\Omega_{1}$ and of $\Omega_{2}$ offered by this Proposition 14.7, we can therefore rewrite the third (still not taken account of) equation (14.4) as

$$
\begin{aligned}
i \Psi \wedge \rho \wedge \kappa- & i R \zeta \wedge \bar{\kappa} \wedge \kappa+(\Pi-\bar{\Pi}) \wedge \rho \wedge \zeta+2 p \kappa \wedge \bar{\kappa} \wedge \zeta-2 \bar{W} \kappa \wedge \bar{\zeta} \wedge \zeta \\
= & -d R \wedge \rho \wedge \zeta-R\left(\pi^{1}+\bar{\pi}^{1}\right) \wedge \rho \wedge \zeta-i R \pi^{2} \wedge \rho \wedge \kappa+i R \kappa \wedge \zeta \wedge \bar{\kappa} \\
& -d J \wedge \rho \wedge \bar{\kappa}-3 J \bar{\pi}^{1} \wedge \rho \wedge \bar{\kappa}-J \rho \wedge \kappa \wedge \bar{\zeta} \\
& -d W \wedge \kappa \wedge \zeta-W \pi^{2} \wedge \rho \wedge \zeta-W \pi^{1} \wedge \kappa \wedge \zeta-W J \rho \wedge \kappa \wedge \bar{\kappa} .
\end{aligned}
$$

But before we commence with analyzing this equation (a long task), we make a side remark. As we can rewrite

$$
\begin{aligned}
& \Omega_{1}=\frac{1}{2}(\Pi+\bar{\Pi}) \wedge \rho+\frac{1}{2}(\Pi-\bar{\Pi}) \wedge \rho+p \kappa \wedge \bar{\kappa}-\bar{W} \kappa \wedge \zeta-W \zeta \wedge \bar{\kappa}, \\
& \Omega_{2}=\Psi \wedge \rho+\frac{1}{2}(\Pi+\bar{\Pi}) \wedge \kappa+\frac{1}{2}(\Pi-\bar{\Pi}) \wedge \kappa-R \zeta \wedge \bar{\kappa},
\end{aligned}
$$

we remark that Proposition 14.5 already tells us that the real part $\frac{1}{2}(\Pi+\bar{\Pi})$ of $\Pi$ is a priori not fully determined, as can be formulated by an

Observation 14.10. For an arbitrary real 1-form $\Lambda$, the 2-forms

$$
\Omega_{1}^{\prime}:=\Omega_{1}+\Lambda \wedge \rho \quad \text { and } \quad \Omega_{2}^{\prime}:=\Omega_{2}+\Lambda \wedge \kappa
$$

still satisfy the structure equations of Proposition 14.1.

Proof. For the sake of completeness, let us detail the arguments. The first equation (14.2) is clear

$$
\left(\Omega_{1}^{\prime}+\bar{\Omega}_{1}^{\prime}\right) \wedge \rho=\left(\Omega_{1}+\underline{\Lambda \wedge \rho} \rho_{\circ}+\bar{\Omega}_{1}+\underline{\Lambda \wedge \rho} \rho_{\circ}\right) \wedge \rho=\left(\Omega_{1}+\bar{\Omega}_{1}\right) \wedge \rho .
$$

The second equation (14.3) also

$$
\begin{aligned}
\Omega_{2}^{\prime} \wedge \rho+\Omega_{1}^{\prime} \wedge \kappa & =\left(\Omega_{2}+\Lambda \wedge \kappa\right) \wedge \rho+\left(\Omega_{1}+\Lambda \wedge \rho\right) \wedge \kappa \\
& =\Omega_{2} \wedge \rho+\underline{\Lambda \wedge \kappa \wedge \rho_{\circ}}+\Omega_{1} \wedge \kappa+\underline{\Lambda \wedge \rho \wedge \kappa} \circ \\
& =\Omega_{2} \wedge \rho+\Omega_{1} \wedge \kappa,
\end{aligned}
$$

and the third one as well

$$
\begin{aligned}
i \Omega_{2}^{\prime} \wedge \kappa+\left(\Omega_{1}^{\prime}-\bar{\Omega}_{1}^{\prime}\right) \wedge \zeta & =i\left(\Omega_{2}+\underline{\Lambda \wedge \kappa_{0}}\right) \wedge \kappa+\left(\Omega_{1}+\underline{\Lambda \wedge \rho} \rho_{\circ}-\bar{\Omega}_{1}-\underline{\Lambda \wedge \rho} \rho_{\circ}\right) \wedge \zeta \\
& =i \Omega_{2} \wedge \kappa+\left(\Omega_{1}-\bar{\Omega}_{1}\right) \wedge \zeta .
\end{aligned}
$$

Now, coming back to (14.9), we remember that we should insert the covariant derivatives $d R$, $d J, d W$ from (14.6), and we will do this in a progressive way, not in one stroke.

Indeed, by wedging $(\bullet) \wedge \rho$ both sides of (14.9), we get rid of $d J, d R$ and it remains only

$$
\begin{aligned}
& -i R \zeta \wedge \bar{\kappa} \wedge \kappa \wedge \rho+2 p \kappa \wedge \bar{\kappa} \wedge \zeta \wedge \rho-2 \bar{W} \kappa \wedge \bar{\zeta} \wedge \zeta \wedge \rho \\
= & i R \kappa \wedge \zeta \wedge \bar{\kappa} \wedge \rho-d W \wedge \kappa \wedge \zeta \wedge \rho-W \pi^{1} \wedge \kappa \wedge \zeta \wedge \rho,
\end{aligned}
$$

that is to say after putting everything to the right

$$
0=-d W \wedge \rho \wedge \kappa \wedge \zeta-(2 p+2 i R) \rho \wedge \kappa \wedge \zeta \wedge \bar{\kappa}+2 \bar{W} p \wedge \kappa \wedge \zeta \wedge \bar{\zeta}-W \pi^{1} \wedge \rho \wedge \kappa \wedge \zeta .
$$


Thus, inserting the expansion of $d W$ from (14.6)

$$
\begin{aligned}
-d W \wedge \rho \wedge \kappa \wedge \zeta= & -W_{\pi^{1}} \pi^{1} \wedge \rho \wedge \kappa \wedge \zeta-W_{\pi^{2}} \pi^{2} \wedge \rho \wedge \kappa \wedge \zeta-W_{\bar{\pi}^{1}} \bar{\pi}^{1} \wedge \rho \wedge \kappa \wedge \zeta \\
& -W_{\bar{\pi}^{2}} \bar{\pi}^{2} \wedge \rho \wedge \kappa \wedge \zeta-W_{\bar{\kappa}} \bar{\kappa} \wedge \rho \wedge \kappa \wedge \zeta-W_{\bar{\zeta}} \bar{\zeta} \wedge \rho \wedge \kappa \wedge \zeta,
\end{aligned}
$$

we get

$$
\begin{aligned}
0= & -\left(W_{\pi^{1}}+W\right) \pi^{1} \wedge \rho \wedge \kappa \wedge \zeta-W_{\pi^{2}} \pi^{2} \wedge \rho \wedge \kappa \wedge \zeta-W_{\bar{\pi}^{1}} \bar{\pi}^{1} \wedge \rho \wedge \kappa \wedge \zeta-W_{\bar{\pi}^{2}} \bar{\pi}^{2} \wedge \rho \wedge \kappa \wedge \zeta \\
& -\left(2 p+2 i R-W_{\bar{\kappa}}\right) \rho \wedge \kappa \wedge \zeta \wedge \bar{\kappa}-\left(2 \bar{W}+W_{\bar{\zeta}}\right) \rho \wedge \kappa \wedge \zeta \wedge \bar{\zeta},
\end{aligned}
$$

whence by identification of coefficients of these independent 4 -forms

$$
\begin{aligned}
& W_{\pi^{1}}=-W, \quad W_{\pi^{2}}=0, \quad W_{\bar{\pi}^{1}}=0, \quad W_{\bar{\pi}^{2}}=0, \\
& W_{\bar{\kappa}}=2 p+2 i R, \quad W_{\bar{\zeta}}=-2 \bar{W},
\end{aligned}
$$

while no condition is imposed so on $W_{\rho}, W_{\kappa}, W_{\zeta}$, and thus

$$
d W=-W \pi^{1}+W_{\rho} \rho+W_{\kappa} \kappa+W_{\zeta} \zeta+(2 p+2 i R) \bar{\kappa}-2 \bar{W} \bar{\zeta} .
$$

Next, putting this expression of $d W$ back into (14.9) allows us to eliminate $p$ so that we can focus only on $\Pi-\bar{\Pi}$ and $\Psi$, which we place on the left

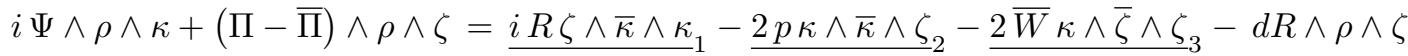

$$
\begin{aligned}
& -R\left(\pi^{1}+\bar{\pi}^{1}\right) \wedge \rho \wedge \zeta-i R \pi^{2} \wedge \rho \wedge \kappa+\underline{i R \kappa \wedge \zeta \wedge \bar{\kappa}_{1}} \\
& -d J \wedge \rho \wedge \bar{\kappa}-3 J \bar{\pi}^{1} \wedge \rho \wedge \bar{\kappa}-J \rho \wedge \kappa \wedge \bar{\zeta} \\
& +\underline{W \pi^{1} \wedge \kappa \wedge \zeta_{4}}-W_{\rho} \rho \wedge \kappa \wedge \zeta-\left(\underline{2}_{2}+\underline{2 i R}_{1}\right) \bar{\kappa} \wedge \kappa \wedge \zeta
\end{aligned}
$$

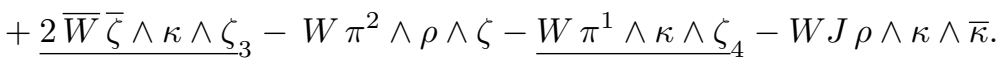

Here, four simplifications by pairs are underlined, in which we observe that $p$ eliminates itself, and if we collect at first the terms divisible by $\rho \wedge \kappa$, we get

$$
\begin{aligned}
i \Psi \wedge \rho \wedge \kappa+(\Pi-\bar{\Pi}) \wedge \rho \wedge \zeta= & \left(-i R \pi^{2}-J \bar{\zeta}-W_{\rho} \zeta-W J \bar{\kappa}\right) \wedge \rho \wedge \kappa \\
& -d R \wedge \rho \wedge \zeta-R\left(\pi^{1}+\bar{\pi}^{1}\right) \wedge \rho \wedge \zeta \\
& -d J \wedge \rho \wedge \bar{\kappa}-3 J \bar{\pi}^{1} \wedge \rho \wedge \bar{\kappa}-W \pi^{2} \wedge \rho \wedge \zeta .
\end{aligned}
$$

By introducing the modified 1-form

$$
\Psi^{\prime}:=\Psi-i\left(i R \pi^{2}+J \bar{\zeta}+W_{\rho} \zeta+W J \bar{\kappa}\right),
$$

the equation becomes

$$
\begin{aligned}
i \Psi^{\prime} \wedge \rho \wedge \kappa+(\Pi-\bar{\Pi}) \wedge \rho \wedge \zeta= & -d R \wedge \rho \wedge \zeta-R\left(\pi^{1}+\bar{\pi}^{1}\right) \wedge \rho \wedge \zeta \\
& -d J \wedge \rho \wedge \bar{\kappa}-3 J \bar{\pi}^{1} \wedge \rho \wedge \bar{\kappa}-W \pi^{2} \wedge \rho \wedge \zeta
\end{aligned}
$$

Now, let us wedge $(\bullet) \wedge \kappa \wedge \zeta$ all this to make $\Psi$ and $\Pi-\bar{\Pi}$ disappear, replacing simultaneously

$$
d J=J_{\pi^{1}} \pi^{1}+J_{\pi^{2}} \pi^{2}+J_{\bar{\pi}^{1}} \bar{\pi}^{1}+J_{\bar{\pi}^{2}} \bar{\pi}^{2}+J_{\rho} \rho+J_{\kappa} \kappa+J_{\zeta} \zeta+J_{\bar{\kappa}} \bar{\kappa}+J_{\bar{\zeta}} \bar{\zeta},
$$

to obtain

$$
\begin{gathered}
\begin{array}{c}
0-J_{\pi^{1}} \pi^{1} \wedge \rho \wedge \bar{\kappa} \wedge \kappa \wedge \zeta-J_{\pi^{2}} \pi^{2} \wedge \rho \wedge \bar{\kappa} \wedge \kappa \wedge \zeta-J_{\bar{\pi}^{1}} \bar{\pi}^{1} \wedge \rho \wedge \bar{\kappa} \wedge \kappa \wedge \zeta-J_{\bar{\pi}^{2}} \bar{\pi}^{2} \wedge \rho \wedge \bar{\kappa} \wedge \kappa \wedge \zeta \\
-J_{\bar{\zeta}} \bar{\zeta} \wedge \rho \wedge \bar{\kappa} \wedge \kappa \wedge \zeta-3 J \bar{\pi}^{1} \wedge \rho \wedge \bar{\kappa} \wedge \kappa \wedge \zeta \\
=-J_{\pi^{1}} \pi^{1} \wedge \rho \wedge \kappa \wedge \zeta \wedge \bar{\kappa}-J_{\pi^{2}} \pi^{2} \wedge \rho \wedge \kappa \wedge \zeta \wedge \bar{\kappa}-\left(J_{\bar{\pi}^{1}}+3 J\right) \bar{\pi}^{1} \wedge \rho \wedge \kappa \wedge \zeta \wedge \bar{\kappa}-J_{\bar{\pi}^{2}} \bar{\pi}^{2} \wedge \rho \wedge \kappa \wedge \zeta \wedge \bar{\kappa} \\
-J_{\bar{\zeta}} \rho \wedge \kappa \wedge \zeta \wedge \bar{\kappa} \wedge \bar{\zeta}
\end{array}
\end{gathered}
$$


and since these 5 -forms are linearly independent, we get by identification

$$
J_{\pi^{1}}=0, \quad J_{\pi^{2}}=0, \quad J_{\bar{\pi}^{1}}=-3 J, \quad J_{\bar{\pi}^{2}}=0, \quad J_{\bar{\zeta}}=0,
$$

while no condition is imposed in this way on $J_{\rho}, J_{\kappa}, J_{\zeta}, J_{\bar{\kappa}}$. Consequently, the 1-form $d J$ contracts as

$$
d J=-3 J \bar{\pi}^{1}+J_{\rho} \rho+J_{\kappa} \kappa+J_{\zeta} \zeta+J_{\bar{\kappa}} \bar{\kappa},
$$

hence putting this expression back into (14.11), we obtain

$i \Psi^{\prime} \wedge \rho \wedge \kappa+(\Pi-\bar{\Pi}) \wedge \rho \wedge \zeta=-d R \wedge \rho \wedge \zeta-R\left(\pi^{1}+\bar{\pi}^{1}\right) \wedge \rho \wedge \zeta$

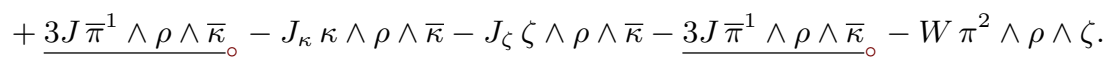

We can yet absorb in $\Psi^{\prime}$ one term from the right-hand side by introducing

$$
\Psi^{\prime \prime}:=\Psi^{\prime}+i J_{\kappa} \bar{\kappa},
$$

so that our equation becomes

$$
\begin{aligned}
i \Psi^{\prime \prime} \wedge \rho \wedge \kappa+(\Pi-\bar{\Pi}) \wedge \rho \wedge \zeta= & -d R \wedge \rho \wedge \zeta-R\left(\pi^{1}+\bar{\pi}^{1}\right) \wedge \rho \wedge \zeta \\
& +J_{\zeta} \rho \wedge \zeta \wedge \bar{\kappa}-W \pi^{2} \wedge \rho \wedge \zeta .
\end{aligned}
$$

Now, observe that all terms except the first one $i \Psi^{\prime \prime} \wedge \rho \wedge \kappa$ are multiple of $\rho \wedge \zeta$. Consequently, wedging on both sides by $(\bullet) \wedge \zeta$, we annihilate everything except

$$
i \Psi^{\prime \prime} \wedge \rho \wedge \kappa \wedge \zeta=0 .
$$

Thanks to the Cartan Lemma, there exist function $e, f, g$ so that

$$
\Psi^{\prime \prime}=e \rho+f \kappa+g \zeta .
$$

For later use, we also observe in passing that

$$
\begin{aligned}
\Psi & =\Psi^{\prime}+i W_{\rho} \zeta+i W J \bar{\kappa}-R \pi^{2}+i J \bar{\zeta} \\
& =\Psi^{\prime \prime}-i J_{\kappa} \bar{\kappa}+i W_{\rho} \zeta+i W J \bar{\kappa}-R \pi^{2}+i J \bar{\zeta} \\
& =-R \pi^{2}+e \rho+f \kappa+\left(i W_{\rho}+g\right) \zeta+i\left(W J-J_{\kappa}\right) \bar{\kappa}+i J \bar{\zeta} .
\end{aligned}
$$

Inserting this just above conducts to an identity

$$
\begin{aligned}
i g \rho \wedge \kappa \wedge \zeta+(\Pi-\bar{\Pi}) \wedge \rho \wedge \zeta= & -d R \wedge \rho \wedge \zeta-R\left(\pi^{1}+\bar{\pi}^{1}\right) \wedge \rho \wedge \zeta \\
& +J_{\zeta} \rho \wedge \zeta \wedge \bar{\kappa}-W \pi^{2} \wedge \rho \wedge \zeta,
\end{aligned}
$$

in which all terms are now multiples of $\rho \wedge \zeta$. Consequently, the Cartan Lemma implies the existence of functions $r$ and $s$ such that

$$
\Pi-\bar{\Pi}=i g \kappa-d R-R \pi^{1}-R \bar{\pi}^{1}+J_{\zeta} \bar{\kappa}-W \pi^{2}+r \rho+s \zeta .
$$

But here, we can take advantage of the fact that $\Pi-\bar{\Pi}$ is purely imaginary to obtain some information about $g, r, s$. Indeed, conjugating

$$
\bar{\Pi}-\Pi=-i \bar{g} \bar{\kappa}-d R-R \bar{\pi}^{1}-R \pi^{1}-\overline{J_{\zeta}} \kappa-\bar{W} \bar{\pi}^{2}+\bar{r} \rho+\bar{s} \bar{\zeta},
$$

and summing, we eliminate $\Pi-\bar{\Pi}$, hence we are left after reorganization with

$$
\begin{aligned}
0= & -2 d R-2 R \pi^{1}-W \pi^{2}-2 R \bar{\pi}^{1}-\bar{W} \bar{\pi}^{2} \\
& +(r+\bar{r}) \rho+\left(i g+\overline{J_{\zeta}}\right) \kappa+s \zeta+\left(-i \bar{g}+J_{\zeta}\right) \bar{\kappa}+\bar{s} \bar{\zeta}
\end{aligned}
$$

Naturally, one has to use the expansion of $d R$ from (14.6) to continue the computation

$$
\begin{aligned}
0= & -\left(2 R_{\pi^{1}}+2 R\right) \pi^{1}-\left(2 R_{\pi^{2}}+W\right) \pi^{2}-\left(2 R_{\bar{\pi}^{1}}+2 R\right) \bar{\pi}^{1}-\left(2 R_{\bar{\pi}^{2}}+\bar{W}\right) \bar{\pi}^{2} \\
& -\left(2 R_{\rho}-r-\bar{r}\right) \rho-\left(2 R_{\kappa}-i g-\overline{J_{\zeta}}\right) \kappa-\left(2 R_{\zeta}-s\right) \zeta-\left(2 R_{\bar{\kappa}}+i \bar{g}-J_{\zeta}\right) \bar{\kappa}-\left(2 R_{\bar{\zeta}}-\bar{s}\right) \bar{\zeta} .
\end{aligned}
$$


An identification to zero of all the nine coefficients of $\pi^{1}, \pi^{2}, \bar{\pi}^{1}, \bar{\pi}^{2}, \rho, \kappa, \zeta, \bar{\kappa}, \bar{\zeta}$ gives

$$
\begin{aligned}
& R_{\pi^{1}}=-R, \quad R_{\pi^{2}}=-\frac{1}{2} W, \quad R_{\bar{\pi}^{1}}=-R, \quad R_{\bar{\pi}^{2}}=-\frac{1}{2} \bar{W}, \\
& R_{\rho}=\frac{1}{2}(r+\bar{r}), \quad R_{\kappa}=\frac{1}{2}\left(i g+\overline{J_{\zeta}}\right), \quad R_{\zeta}=\frac{1}{2} s, \quad R_{\bar{\kappa}}=\frac{1}{2}\left(-i \bar{g}+J_{\zeta}\right), \quad R_{\bar{\zeta}}=\frac{1}{2} \bar{s},
\end{aligned}
$$

and so:

$$
d R=-R \pi^{1}-\frac{1}{2} W \pi^{2}-R \bar{\pi}^{1}-\frac{1}{2} \bar{W} \bar{\pi}^{2}+R_{\rho} \rho+R_{\kappa} \kappa+R_{\zeta} \zeta+R_{\bar{\kappa}} \bar{\kappa}+R_{\bar{\zeta}} \bar{\zeta} .
$$

Inserting this back into what precedes, we can therefore obtain both

$$
\Pi-\bar{\Pi}=-\frac{1}{2} W \pi^{2}+\frac{1}{2} \bar{W} \bar{\pi}^{2}+R_{\zeta} \zeta-R_{\bar{\zeta}} \bar{\zeta}+\left(R_{\kappa}-\overline{J_{\zeta}}\right) \kappa-\left(R_{\bar{\kappa}}-J_{\zeta}\right) \bar{\kappa}+\frac{1}{2}\left(g_{\rho}-\bar{g}_{\rho}\right) \rho,
$$

and replacing $g=-2 i R_{\kappa}+i \overline{J_{\zeta}}$ in (14.12)

$$
\Psi=-R \pi^{2}+e \rho+f \kappa+i\left(W_{\rho}-2 R_{\kappa}+\overline{J_{\zeta}}\right) \zeta+i\left(W J-J_{\kappa}\right) \bar{\kappa}+i J \bar{\zeta} .
$$

Thus

$$
\begin{aligned}
\Omega_{1}= & p \kappa \wedge \bar{\kappa}+\Pi \wedge \rho+\overline{W \zeta} \wedge \kappa-W \zeta \wedge \bar{\kappa} \\
= & p \kappa \wedge \bar{\kappa}+\frac{1}{2}(\Pi-\bar{\Pi}) \wedge \rho+\overline{W \zeta} \wedge \kappa-W \zeta \wedge \bar{\kappa}+\frac{1}{2}(\Pi+\bar{\Pi}) \wedge \rho \\
= & -\frac{1}{4} W \pi^{2} \wedge \rho+\frac{1}{4} \bar{W} \bar{\pi}^{2} \wedge \rho-\frac{1}{2}\left(R_{\kappa}-\overline{J_{\zeta}}\right) \rho \wedge \kappa-\frac{1}{2} R_{\zeta} \rho \wedge \zeta+\frac{1}{2}\left(R_{\bar{\kappa}}-J_{\zeta}\right) \rho \wedge \bar{\kappa} \\
& \quad+\frac{1}{2} R_{\bar{\zeta}} \rho \wedge \bar{\zeta}+\left(\frac{1}{2} W_{\bar{\kappa}}-i R\right) \kappa \wedge \bar{\kappa}-\bar{W} \kappa \wedge \bar{\zeta}-W \zeta \wedge \bar{\kappa}+\frac{1}{2}(\Pi+\bar{\Pi}) \wedge \rho,
\end{aligned}
$$

and

$$
\begin{aligned}
\Omega_{2}= & -R \pi^{2} \wedge \rho-\frac{1}{4} W \pi^{2} \wedge \kappa+\frac{1}{4} \bar{W} \bar{\pi}^{2} \wedge \kappa-i\left(W_{\rho}-2 R_{\kappa}+\overline{J_{\zeta}}\right) \rho \wedge \zeta \\
& -i\left(W J-J_{\kappa}\right) \rho \wedge \bar{\kappa}-i J \rho \wedge \bar{\zeta}-\frac{1}{2} R_{\zeta} \kappa \wedge \zeta+\frac{1}{2}\left(R_{\bar{\kappa}}-J_{\zeta}\right) \kappa \wedge \bar{\kappa}+\frac{1}{2} R_{\bar{\zeta}} \kappa \wedge \bar{\zeta} \\
& -R \zeta \wedge \bar{\kappa}+\frac{1}{2}(\Pi+\bar{\Pi}) \wedge \kappa+\left(\frac{1}{2}(r-\bar{r})-f\right) \rho \wedge \kappa .
\end{aligned}
$$

If we define

$$
\Lambda:=\frac{1}{2}(\Pi+\bar{\Pi})+\text { real part of }\left(\frac{1}{2}\left(g_{\rho}-\overline{g_{\rho}}\right)-d_{\kappa}\right) \rho
$$

and

$$
h:=\text { imaginary part of }\left(\frac{1}{2}\left(g_{\rho}-\overline{g_{\rho}}\right)-d_{\kappa}\right) \text {, }
$$

we conclude that

$$
\begin{aligned}
\Omega_{1}= & -\frac{1}{4} W \pi^{2} \wedge \rho+\frac{1}{4} \bar{W} \bar{\pi}^{2} \wedge \rho-\frac{1}{2}\left(R_{\kappa}-\overline{J_{\zeta}}\right) \rho \wedge \kappa-\frac{1}{2} R_{\zeta} \rho \wedge \zeta+\frac{1}{2}\left(R_{\bar{\kappa}}-J_{\zeta}\right) \rho \wedge \bar{\kappa} \\
& +\frac{1}{2} R_{\bar{\zeta}} \rho \wedge \bar{\zeta}+\left(\frac{1}{2} W_{\bar{\kappa}}-i R\right) \kappa \wedge \bar{\kappa}-\bar{W} \kappa \wedge \bar{\zeta}-W \zeta \wedge \bar{\kappa}+\Lambda \wedge \rho, \\
\Omega_{2}= & -R \pi^{2} \wedge \rho-\frac{1}{4} W \pi^{2} \wedge \kappa+\frac{1}{4} \bar{W} \bar{\pi}^{2} \wedge \kappa-i\left(W_{\rho}-2 R_{\kappa}+\overline{J_{\zeta}}\right) \rho \wedge \zeta \\
& -i\left(W J-J_{\kappa}\right) \rho \wedge \bar{\kappa}-i J \rho \wedge \bar{\zeta}-\frac{1}{2} R_{\zeta} \kappa \wedge \zeta+\frac{1}{2}\left(R_{\bar{\kappa}}-J_{\zeta}\right) \kappa \wedge \bar{\kappa}+\frac{1}{2} R_{\bar{\zeta}} \kappa \wedge \bar{\zeta} \\
& -R \zeta \wedge \bar{\kappa}+\Lambda \wedge \kappa+h \rho \wedge \kappa .
\end{aligned}
$$

Notice that all coefficients of 2-forms - except only $h$ - depend on $R, J, W$ and their coframe derivatives. 
We are now close to the termination towards an $\{e\}$-structure. In summary, we have obtained the following structure equations

$$
\begin{aligned}
d \rho= & \pi^{1} \wedge \rho+\bar{\pi}^{1} \wedge \rho+i \kappa \wedge \bar{\kappa} \\
d \kappa= & \pi^{1} \wedge \kappa+\pi^{2} \wedge \rho+\zeta \wedge \bar{\kappa} \\
d \zeta= & i \pi^{2} \wedge \kappa+\pi^{1} \wedge \zeta-\bar{\pi}^{1} \wedge \zeta+W \kappa \wedge \zeta+R \rho \wedge \zeta+J \rho \wedge \bar{\kappa}, \\
d \pi^{1}= & \Lambda \wedge \rho-\frac{1}{4} W \pi^{2} \wedge \rho+\frac{1}{4} \overline{W^{2}} \bar{\pi}^{2} \wedge \rho-i \bar{\pi}^{2} \wedge \kappa \\
& -\frac{1}{2}\left(R_{\kappa}-\overline{J_{\zeta}}\right) \rho \wedge \kappa-\frac{1}{2} R_{\zeta} \rho \wedge+\frac{1}{2}\left(R_{\bar{\kappa}}-J_{\zeta}\right) \rho \wedge \bar{\kappa}+\frac{1}{2} R_{\bar{\zeta}} \rho \wedge \bar{\zeta} \\
& +\left(\frac{1}{2} W_{\bar{\kappa}}-i R\right) \kappa \wedge \bar{\kappa}-\bar{W} \kappa \wedge \bar{\zeta}-W \zeta \wedge \bar{\kappa}+\zeta \wedge \bar{\zeta} \\
d \pi^{2}= & \Lambda \wedge \kappa+\pi^{2} \wedge \bar{\pi}^{1}-\bar{\pi}^{2} \wedge \zeta-R \pi^{2} \wedge \rho-\frac{1}{4} W \pi^{2} \wedge \kappa+\frac{1}{4} \bar{W} \bar{\pi}^{2} \wedge \kappa \\
& +h \rho \wedge \kappa-i\left(W_{\rho}-2 R_{\kappa}+\overline{J_{\zeta}}\right) \rho \wedge \zeta-i\left(W J-J_{\kappa}\right) \rho \wedge \bar{\kappa}-i J \rho \wedge \bar{\zeta} \\
& -\frac{1}{2} R_{\zeta} \kappa \wedge \zeta+\frac{1}{2}\left(R_{\bar{\kappa}}-J_{\zeta}\right) \kappa \wedge \bar{\kappa}+\frac{1}{2} R_{\bar{\zeta}} \kappa \wedge \bar{\zeta}-R \zeta \wedge \bar{\kappa} .
\end{aligned}
$$

But at this stage, we cannot directly deduce from these equations an appropriate expression for $h$. For example, any attempt to isolate $h$ by wedging the equation $d \pi^{2}=\cdots$ with any appropriate differential form will include a component of Maurer-Cartan type. This is to be expected, because $h$ will soon be shown below to depend on higher order jets of $R, J, W$, while the torsions above only depend up to the $2^{\text {nd }}$-order jets of these invariants. Therefore, an application of the exterior differentiation on both sides of the equation $d \pi^{2}=\cdots$ appears necessary to reach an expression for $h$ from the Poincaré relation $d \circ d=0$.

To facilitate the discussion, we set

$$
\begin{aligned}
\widehat{\Omega}_{1}= & -\frac{1}{4} W \pi^{2} \wedge \rho+\frac{1}{4} \bar{W} \bar{\pi}^{2} \wedge \rho-\frac{1}{2}\left(R_{\kappa}-\overline{J_{\zeta}}\right) \rho \wedge \kappa-\frac{1}{2} R_{\zeta} \rho \wedge \zeta \\
& +\frac{1}{2}\left(R_{\bar{\kappa}}-J_{\zeta}\right) \rho \wedge \bar{\kappa}+\frac{1}{2} R_{\bar{\zeta}} \rho \wedge \bar{\zeta}+\left(\frac{1}{2} W_{\bar{\kappa}}-i R\right) \kappa \wedge \bar{\kappa}-\bar{W} \kappa \wedge \bar{\zeta}-W \zeta \wedge \bar{\kappa}, \\
\widehat{\Omega}_{2}= & -R \pi^{2} \wedge \rho-\frac{1}{4} W \pi^{2} \wedge \kappa+\frac{1}{4} \bar{W} \bar{\pi}^{2} \wedge \kappa-i\left(W_{\rho}-2 R_{\kappa}+\overline{J_{\zeta}}\right) \rho \wedge \zeta \\
& -i\left(W J-J_{\kappa}\right) \rho \wedge \bar{\kappa}-i J \rho \wedge \bar{\zeta}-\frac{1}{2} R_{\zeta} \kappa \wedge \zeta+\frac{1}{2}\left(R_{\bar{\kappa}}-J_{\zeta}\right) \kappa \wedge \bar{\kappa}+\frac{1}{2} R_{\bar{\zeta}} \kappa \wedge \bar{\zeta} \\
& -R \zeta \wedge \bar{\kappa},
\end{aligned}
$$

so that

$$
\begin{aligned}
& d \pi^{1}=\Lambda \wedge \rho-i \bar{\pi}^{2} \wedge \kappa+\zeta \wedge \bar{\zeta}+\widehat{\Omega}_{1}, \\
& d \pi^{2}=\Lambda \wedge \kappa+\pi^{2} \wedge \bar{\pi}^{1}-\bar{\pi}^{2} \wedge \zeta+\widehat{\Omega}_{2}+h \rho \wedge \kappa .
\end{aligned}
$$

Proposition 14.13. The function $h$ is a function of the $3^{\text {rd }}$-order jets of $W$ and $J$.

Proof. By applying exterior differentiation $d$ to the equation of $d \pi^{2}$, while wedging on both sides with $\kappa \wedge \pi^{1} \wedge \bar{\pi}^{1} \wedge \pi^{2} \wedge \bar{\pi}^{2}$, we obtain

$$
\begin{aligned}
2 h \rho \wedge \kappa \wedge \bar{\kappa} \wedge \zeta \wedge \pi^{1} \wedge \bar{\pi}^{1} \wedge \pi^{2} \wedge \bar{\pi}^{2}= & -\overline{\widehat{\Omega}}_{2} \wedge \kappa \wedge \zeta \wedge \pi^{1} \wedge \bar{\pi}^{1} \wedge \pi^{2} \wedge \bar{\pi}^{2} \\
& -d \widehat{\Omega}_{2} \wedge \kappa \wedge \pi^{1} \wedge \bar{\pi}^{1} \wedge \pi^{2} \wedge \bar{\pi}^{2}
\end{aligned}
$$

At this point, let $\Phi$ be the auxiliary real 2-form

$$
\Phi:=d \Lambda-\Lambda \wedge \pi^{1}-\Lambda \wedge \bar{\pi}^{1}-i \pi^{2} \wedge \bar{\pi}^{2} .
$$


Again this comes from the consideration of the model case. The structure equations therefore become

$$
\begin{aligned}
d \rho & =\pi^{1} \wedge \rho+\bar{\pi}^{1} \wedge \rho+i \kappa \wedge \bar{\kappa} \\
d \kappa & =\pi^{1} \wedge \kappa+\pi^{2} \wedge \rho+\zeta \wedge \bar{\kappa} \\
d \zeta & =i \pi^{2} \wedge \kappa+\pi^{1} \wedge \zeta-\bar{\pi}^{1} \wedge \zeta+W \kappa \wedge \zeta+R \rho \wedge \zeta+J \rho \wedge \bar{\kappa} \\
d \pi^{1} & =\Lambda \wedge \rho-i \bar{\pi}^{2} \wedge \kappa+\zeta \wedge \bar{\zeta}+\widehat{\Omega}_{1} \\
d \pi^{2} & =\Lambda \wedge \kappa+\pi^{2} \wedge \bar{\pi}^{1}-\bar{\pi}^{2} \wedge \zeta+\widehat{\Omega}_{2}+h \rho \wedge \kappa \\
d \Lambda & =\Lambda \wedge \pi^{1}+\Lambda \wedge \bar{\pi}^{1}+i \pi^{2} \wedge \bar{\pi}^{2}+\Phi .
\end{aligned}
$$

Proposition 14.14. The real 2 -form $\Phi$ is a function of the $4^{\text {th }}$-order jets of $W$ and $J$.

Proof. By taking exterior derivative of $d \pi^{1}$ and $d \pi^{2}$ again, this time using the expression of $d \Lambda$, we have

$$
\begin{aligned}
\Phi \wedge \rho= & i \bar{\Omega}_{2} \wedge \kappa+i h \rho \wedge \kappa \wedge \bar{\kappa}-W \kappa \wedge \zeta \wedge \bar{\zeta}+\bar{W} \zeta \wedge \bar{\kappa} \wedge \bar{\zeta}-2 R \rho \wedge \zeta \wedge \bar{\zeta} \\
& -J \rho \wedge \bar{\kappa} \wedge \bar{\zeta}+\bar{J} \rho \wedge \kappa \wedge \zeta-d \widehat{\Omega}_{1}, \\
\Phi \wedge \kappa=- & \widehat{\Omega}_{2} \wedge \bar{\pi}^{1}-h \rho \wedge \kappa \wedge \bar{\pi}^{1}+\pi^{2} \wedge \overline{\widehat{\Omega}}_{1}+\overline{\widehat{\Omega}}_{2} \wedge \zeta-h \rho \wedge \bar{\kappa} \wedge \zeta-W \bar{\pi}^{2} \wedge \kappa \wedge \zeta \\
& -R \bar{\pi}^{2} \wedge \rho \wedge \zeta-J \bar{\pi}^{2} \wedge \rho \wedge \bar{\kappa}-d \widehat{\Omega}_{2}-d(h \rho \wedge \kappa) .
\end{aligned}
$$

Writing $\Phi$ as

$$
\Phi=\widehat{\Omega}_{3}+u \rho \wedge \kappa,
$$

where $\widehat{\Omega}_{3}$ is the 2-form not containing $\rho \wedge \kappa$, then each of the coefficients in $\widehat{\Omega}_{3}$ is a function of the $4^{\text {th }}$-order jet of $W$ and $J$. Since $\Phi$ is real, taking conjugate on both sides, we must have

$$
\widehat{\Omega}_{3}+u \rho \wedge \kappa=\overline{\widehat{\Omega}}_{3}+\bar{u} \rho \wedge \bar{\kappa} .
$$

Therefore by inspection, $\bar{u}$ is also a function of the $4^{\text {th }}$-order jets of $W$ and $J$, and therefore so is $u$. This finishes the proof.

With this proposition, we have therefore fully constructed an $\{e\}$-structure.

\section{ACKNOWLEDGEMENTS}

Both authors benefited from enlightening exchanges with Paweł Nurowski.

This research was supported in part by the Polish National Science Centre (NCN) via the grant number 2018/29/B/ST1/02583, and by the Norwegian Financial Mechanism 2014-2021 via the project registration number 2019/34/H/ST1/00636.

\section{REFERENCES}

[1] Z. Chen, W. G. Foo, J. Merker and T. A. Ta: Normal forms for rigid $\mathfrak{C}_{2,1}$ hypersurfaces $M^{5} \subset \mathbb{C}^{3}$, Taiwanese Journal of Mathematics, 25 (2) (2021), 333-364, arxiv.org/abs/1912.01655/

[2] Z. Chen, J. Merker: On differential invariants of parabolic surfaces, Dissertationes Mathematicæ, 559 (2021), 110 pages, arxiv.org/abs/1908.07867/

[3] M. Fels, W. Kaup: CR manifolds of dimension 5: a Lie algebra approach, J. Reine Angew. Math., 604 (2007), $47-71$.

[4] M. Fels, W. Kaup: Classification of Levi degenerate homogeneous CR-manifolds in dimension 5, Acta Math., 201 (2008), $1-82$.

[5] W. G. Foo, J. Merker and T. A. Ta: Rigid equivalences of 5-dimensional 2-nondegenerate rigid real hypersurfaces $M^{5} \subset \mathbb{C}^{3}$ of constant Levi rank 1, Michigan Math. J., to appear, arxiv.org/abs/1904.02562/.

[6] M. Freeman: Real submanifolds with degenerate Levi form. Several complex variables (Proc. Sympos. Pure Math., Vol. XXX, Williams Coll., Williamstown, Mass., 1975), Part 1, pp. 141-147. Amer. Math. Soc., Providence, R.I., 1977. 
[7] M. Freeman: Local biholomorphic straightening of real submanifolds, Annals of Mathematics, 106 (2) (1977), 319-352.

[8] H. Gaussier, J. Merker: A new example of uniformly Levi degenerate hypersurface in $\mathbb{C}^{3}$, Ark. Mat., 41 (1) (2003), 85-94. Erratum: 45 (2) (2007), 269-271.

[9] A. Isaev: Zero CR-Curvature Equations for Levi Degenerate Hypersurfaces via Pocchiola's Invariant, arxiv.org/pdf/1809.03029/

[10] H. Jacobowitz: An introduction to CR structures, Math. Surveys and Monographs, 32, Amer. Math. Soc., Providence (1990).

[11] S. Lie (Author), J. Merker (Editor): Theory of Transformation Groups I. General Properties of Continuous Transformation Groups. A Contemporary Approach and Translation, Springer-Verlag, Berlin, Heidelberg (2015), arxiv.org/abs/1003.3202/

[12] C. Medori, A. Spiro: The equivalence problem for 5-dimensional Levi degenerate CR manifolds, Int. Math. Res. Not. IMRN, 2014 (20), 5602-5647.

[13] C. Medori, A. Spiro: Structure equations of Levi degenerate CR hypersurfaces of uniform type, Rend. Semin. Mat. Univ. Politec. Torino, 73 (1-2) (2015), 127-150.

[14] J. Merker, P. Nurowski: On degenerate para-CR structures: Cartan reduction and homogeneous models, arxiv.org/abs/2003.08166/ (2020).

[15] J. Merker: Lie symmetries of partial differential equations and CR geometry, Journal of Mathematical Sciences (N.Y.), 154 (2008), 817-922.

[16] J. Merker: Equivalences of 5-dimensional CR manifolds, IV: Six ambiguity matrix groups (Initial G-structures), arxiv.org/abs/1312.1084/

[17] J. Merker: Equivalences of 5-dimensional CR-manifolds V: Six initial frames and coframes; Explicitness obstacles, arxiv.org/abs/1312.3581/

[18] J. Merker, S. Pocchiola: Explicit absolute parallelism for 2-nondegenerate real hypersurfaces $M^{5} \subset \mathbb{C}^{3}$ of constant Levi rank 1, Journal of Geometric Analysis, 30 (2020), 2689-2730, 10.1007/s12220-018-9988-3. Addendum: 3233-3242, 10.1007/s12220-019-00195-2.

[19] J. Merker, S. Pocchiola and M. Sabzevari: Equivalences of 5-dimensional CR manifolds, II: General classes I, II, III 1 , III $\mathrm{IV}_{1}, \mathrm{IV}_{2}, 5$ figures, 95 pages, arxiv.org/abs/1311.5669/

[20] P. Nurowski, J. Tafel: Symmetries of Cauchy-Riemann spaces, Lett. Math. Phys., 15 (1) (1988), 31-38.

[21] P. J. Olver: Equivalence, Invariants, and Symmetry, Cambridge University Press, Cambridge, (1995).

[22] P. J. Olver: Normal forms for submanifolds under group actions, Symmetries, differential equations and applications, 1-25. Springer Proc. Math. Stat. 266, Springer, Cham, (2018).

[23] P. J. Olver, J. Pohjanpelto: Moving frames for Lie pseudo-groups, Canad. J. Math., 60 (6) (2008), 1336-1386.

[24] P. J. Olver, J. Pohjanpelto: Differential invariant algebras of Lie pseudo-groups, Adv. Math., 222 (5) (2009), 1746-1792.

[25] S. Pocchiola: Explicit absolute parallelism for 2-nondegenerate real hypersurfaces $M^{5} \subset \mathbb{C}^{3}$ of constant Levi rank 1 , arxiv.org/abs/1312.6400/

\section{WEI GUO FOO}

ACADEMIA SINICA

INSTITUTE OF MATHEMATICS

TAIPEI, TAIWAN

ORCID: 0000-0002-9022-8177

E-mail address: fooweiguo@gate.sinica.edu.tw

JOËL MERKER

UNIVERSITÉ PARIS-SACLAY

LABORATOIRE DE MATHÉMATIQUES D'ORSAY

CNRS, 91405 ORSAY CEDEX, FRANCE,

ORCID: 0000-0003-2653-2147

E-mail address: joel.merker@universite-paris-saclay.fr 